\title{
A Comprehensive Spectral and Variability Study of Narrow-Line Seyfert 1 Galaxies Observed by ASCA: II. Spectral Analysis and Correlations
}

\author{
Karen M. Leighly \\ Columbia Astrophysics Laboratory, 550 West 120th Street, New York, NY 10027, USA, \\ leighly@ulisse.phys.columbia.edu
}

\begin{abstract}
I present a comprehensive and uniform analysis of $25 A S C A$ observations from 23 Narrow-line Seyfert 1 galaxies. The spectral analysis and correlations are presented in this paper, Part 2; the reduction and time series analysis is presented in the companion paper, Part 1.

A maximum likelihood analysis confirms that the hard X-ray photon index is significantly steeper at $>90 \%$ confidence in this sample of NLS1s compared with a random sample of Seyfert galaxies with broad optical lines. Soft excess emission was detected in 17 of the 19 objects which had no significant absorption, a result that demonstrates that soft excesses appear considerably more frequently in NLS1s than in Seyfert 1 galaxies with broad optical lines. The strength of the soft excess, parameterized using a flux ratio obtained from the black body plus power law model, has a wide range of values in these objects (a factor of 50). The photon index was found to be correlated with the $\mathrm{H} \beta$ FWHM, despite the small range of the latter parameter; however, neither parameter is correlated with the strength of the soft excess or ROSAT slope. Therefore, assuming that an excess of soft photons results in the steep photon index and narrow $\mathrm{H} \beta$ FWHM, that excess may lie primarily in the unobservable EUV. The strength of the soft excess is correlated with the variability parameters, so that objects with strong soft excesses show higher amplitude variability; this potentially important result is not easily explained. While a range of two orders of magnitude in luminosity is represented, the temperature of the soft excess is approximately consistent throughout the sample, in contrast with expectations of simple accretion disk models. The presence of ionized absorption was sought using a two-edge model. It was found that this component appears to be typically less common in NLS1s and some evidence was found that the typical ionization state is lower compared with broad-line Seyfert galaxies. This fact, plus evidence for a correlation between the presence of the warm absorber and significant optical polarization, may imply that the inner warm absorber is missing or is too highly ionized to be detected, and only the outer, dusty, less ionized warm absorber is present in many cases. The iron line equivalent width appears to be similar among narrow and broad-line Seyfert galaxies. This could mean that reprocessing occurs with similar geometry in both classes of objects; however, the detection of ionized iron lines in a few objects, implying possibly altered fluorescence
\end{abstract}


yields, and poor statistics, makes this conclusion tentative. Constraints on physical processes and models based on extreme values of orientation and accretion rate for NLS1s are examined in light of the observational results.

Subject headings: galaxies: active — galaxies: Seyfert $-\mathrm{X}$-rays: galaxies 


\section{Introduction}

Narrow-line Seyfert galaxies are identified by their optical line properties: H $\beta$ FWHM is $<2000 \mathrm{~km} / \mathrm{s}$, the [O III] $\lambda 5007$ to $\mathrm{H} \beta$ ratio is $<3$ and there are high ionization lines and frequently strong Fe II emission present in the spectrum (Osterbrock \& Pogge 1985; Goodrich 1989). Although their permitted lines may be only slightly broader than their forbidden lines, they can be clearly distinguished from Seyfert 2 galaxies. For example, the permitted lines are polarized differently than the forbidden lines, indicating two emission regions (Goodrich 1989). As shown by Boroson \& Green (1992) these emission line properties are strongly correlated; a principal component analysis (PCA) shows that much of the variance in line properties can be traced to a single eigenvector, despite the fact that different emission lines are thought to originate in widely separated parts of the AGN. Therefore, these correlations must have an origin in a primary intrinsic physical parameter. The narrow-line Seyfert 1 galaxies are located at the extreme end of the Boroson and Green eigenvector; therefore, as a class they exemplify an extreme value of this physical parameter. It is important to identify this physical parameter and to understand how it drives these correlations.

This paper is the second of a two part series that presents the first uniform analysis of the X-ray variability and spectral properties from $A S C A$ observations of a sizable sample of Narrow-line Seyfert 1 galaxies. Part 1 presents the data reduction and the time series analysis of the $A S C A$ data as well as a preliminary search for spectral variability and a general introduction to this subclass of Seyfert 1 galaxy. This part presents the spectral analysis of the $A S C A$ data and studies of correlations between the spectral and variability parameters and optical emission line information from the literature as well as from analysis of spectra in hand. The results are discussed in terms of current models for the X-ray emission from NLS1s and Seyfert galaxies; in particular, effects of orientation and accretion rate are discussed. Some of these results have been previously presented in Leighly 1998.

ROSAT observations demonstrated that the soft X-ray spectra from NLS1s is systematically steeper than those from Seyfert 1 galaxies with broad optical lines (Boller, Brandt \& Fink 1996; Forster \& Halpern 1996; Laor et al. 1997a). ASCA spectra from NLS1s have the advantage of a broader band pass and better energy resolution than the ROSAT PSPC spectra. A breakthrough in the understanding of NLS1s occurred with the observation of RE 1034+39 using ASCA (Pounds, Done \& Osborne 1995). The $A S C A$ spectrum revealed a very strong soft excess component dominating the spectrum below 1-2 keV, and a very steep hard X-ray power law with photon index $\sim 2.6$. This spectrum is very different than the typical Seyfert 1 spectrum, which tends to have a flatter power law with photon index typically around 1.7-1.9 and the soft excess is generally not observed in the $A S C A$ band pass. This dichotomy resembles that observed between Galactic black hole candidates (GBHC) in the hard (or low) and soft (or high) states (e.g. Nowak 1995), a fact that prompted Pounds, Done \& Osborne (1995) to postulate that NLS1s are the supermassive black hole analogs of Galactic black hole candidates in the soft state. Soft state GBHC are thought to be accreting at a larger fraction of the Eddington limit than hard state 
GBHC; this analogy supports the idea that overall behavior of NLS1s originates from an accretion rate that is a higher fraction of Eddington than in Seyfert 1 galaxies with broader optical lines. More recently, Brandt, Mathur \& Elvis (1997) presented a collection of photon indices from ASCA observations of Seyfert galaxies obtained from the literature. They found that the steep hard X-ray spectrum from RE $1034+39$ is not anomalous; NLS1s have generally steeper hard (2-10 $\mathrm{keV}$ ) X-ray spectra than broad-line Seyfert 1 galaxies.

Narrow-line Seyfert 1 galaxies have been also observed to exhibit peculiar spectral features in their $A S C A$ spectra that have not been observed in Seyfert 1 galaxies with broad optical lines. Three NLS1s which have very strong soft excesses were discovered to have peculiar absorption features near $1 \mathrm{keV}$ (Leighly et al. 1997a; see also Otani, Kii \& Miya 1996; Hayashida 1996; Comastri, Molendi \& Ulrich 1997). Absorption by highly ionized oxygen is common in Seyfert 1 galaxies with broad optical lines (e.g. Reynolds 1997). However, the energies of the features in these three objects is far too high to be interpreted as absorption by oxygen in the rest frame of the galaxy. Citing the similarities between many aspects of NLS1s and broad-absorption line quasars, which are known for their UV absorption lines that indicate high-velocity outflows, Leighly et al. (1997a) suggested that these features are due to relativistically outflowing gas with speeds of 0.2-0.6 c. More recently, Nicastro, Fiore \& Matt 1999 presented an alternative interpretation: they suggest that these features are due to absorption by large columns of highly ionized gas, and the features are due to resonant absorption primarily by Fe L.

Other unusual features have also been found. Fiore et al. 1998 discovered a feature near $1 \mathrm{keV}$ in the spectrum of PG $1244+026$ that is best modeled by an emission line. Comastri et al. 1998 discovered that the iron line in the BeppoSAX spectrum of Ton S180 has an energy near $6.7 \mathrm{keV}$ indicating that the iron is ionized. Both of these features were interpreted as evidence for reprocessing in an accretion disk which is highly ionized, a property predicted if the accretion rate is high (e.g. Matt, Fabian \& Ross 1993a). However, it has been shown more recently that $6.7 \mathrm{keV}$ iron lines are not restricted to NLS1s but occur also in Seyfert 1 galaxies with broad optical lines (e.g. Guainazzi et al. 1998a).

\section{Data Reduction}

\subsection{Spectra}

The data reduction and filtering are described in Part 1, and this section describes treatment of the data specific to the spectral fitting. Dark-frame error and echo corrections were made on data taken in Faint mode. As the Rev. 2 reprocessing had been performed on all of the data, no corrections for the gain in GIS3 during later time periods was made.

To obtain the best signal to noise, a range of three different-sized extraction regions were used. For brightest objects, the nominal extraction regions ( $4^{\prime}$ and $6^{\prime}$ for SIS and GIS respectively) 
were used. For fainter objects, regions $87.5 \%$ and $75 \%$ of the nominal ones were used. Background spectra were accumulated from source-free parts of the detector.

In order to account for the decline in the energy resolution as a function of time for the CCD detectors, response matrices appropriate for the date of the observation were made using the script sisrmg. Ancillary response files (arf files) were made for each detector using ascaarf. Several early observations were made in 4CCD or 2CCD Bright mode and significant flux is spread out over multiple chips. For the most part, these were reduced and combined as recommended in The ASCA Data Reduction Guide. Spectra and response files were made for each separate chip. Response files were averaged together using addrmf weighted by the number of source photons in each chip. A spectrum was extracted from the entire source region and the arf file was made using that.

Spectral fitting was performed using XSPEC v. 10. The energy bands over which the spectra were fitted varied. For observations before January 1996, the lower limit was constrained only by the presence of a lower level discriminator applied during the observation. In fact, a level discriminator was applied only during the I Zw 1, RX J0439-45, 1H 0707-495 and Ark 564 observations. The GIS spectra were fit above $0.8 \mathrm{keV}$, since the GIS detectors are not well calibrated below that energy. Although Rev. 2 reprocessing should account for the gain changes

in GIS3, better behavior of the iron line was obtained from some spectra when the region around the iron line was excluded from spectral fitting. After January 1996, differences between the SIS0 and SIS1 detectors at low energies are marked. This is thought to be due to Residual Dark Distribution (RDD) effects (Dotani 1998), and this problem most seriously affects the spectra below about $0.8-1.0 \mathrm{keV}$. It is thought that this effect more seriously affects the SIS1 detector because of its average higher temperature. Therefore, during observations from this time period, the SIS1 spectra below $0.8 \mathrm{keV}$ were ignored, and GIS spectra below $1.0 \mathrm{keV}$ were ignored.

The spectra from each observation were fit separately, except for IRAS $13349+2438$. There were two observations of this object, separated by about 4 days. It has previously been shown that no detectable spectral variability had occurred between these two observations (Brinkmann et al. 1996; Brandt et al. 1997); this was verified and the data from these two observations were combined. For statistical tests, the properties of NGC 4051 were taken from the second, longer observation because of the better statistics offered.

\subsection{ROSAT data}

Nearly all of these objects considered here had pointed ROSAT observations. These were reduced using Xselect and fit between $0.1-2 \mathrm{keV}$. The intent of using ROSAT spectra in this paper is to investigate the relationship between the slope of the soft X-ray spectrum and the soft excess observed in the $A S C A$ spectra. The poor energy resolution of ROSAT prevents robust deconvolution of complex models. Therefore, we quote only the results from a power law fit 
with Galactic absorption, or with extra absorption if that improves the fit at greater than $90 \%$ confidence.

Three objects did not have pointed observations: RX J0439-45, PKS 0558-504 and 1H 0707-495. The information for these was taken from the ROSAT All Sky Survey data: for the first two, information from the spectra was available in the literature and for the last one, an estimation was made for the slope using the hardness ratios available in the ROSAT Bright Source Catalog (Grupe 1997 P. comm.). Finally, IRAS 20181-2244 was detected in the ROSAT All Sky Survey (Boller et al. 1992; Boller et al. 1998) but the flux was too low for it to be included in the ROSAT Bright Source Catalog. Therefore, no photon index estimate is possible.

\subsection{Emission Line Data}

Table 1 lists some of the optical emission line properties from NLS1s, obtained both from the literature and from spectra in-hand. The properties include the FWHM of $\mathrm{H} \beta$, the $\mathrm{H} \beta$ equivalent width, the ratio of Fe II to $\mathrm{H} \beta$, the FWHM of [O III] when available, and the ratio of [O III] to $\mathrm{H} \beta$. Measurement of these parameters is complicated by the fact that NLS1s often exhibit strong emission from blends of optical Fe II. This contaminates the continuum around the $\mathrm{H} \beta /[\mathrm{O}$ III] complex and furthermore the multiplet 42 occurs so close to [O III] $\lambda 5007$ that it can significantly distort the profile. One of the more effective ways to deal with this problem is to subtract the Fe II using a scaled and broadened Fe II template formed from the spectrum of the quintessential NLS1 I Zw 1. This method was used in the seminal paper by Boroson \& Green (1992) and has subsequently been used by several other authors including Grupe 1996 (also Grupe et al. 1999a). While the I Zw 1 template method does not provide a perfect iron subtraction (e.g. Boroson \& Green 1992), it is useful also because it provides a consistent way to estimate the contribution of Fe II in the spectra. Seeking consistency in Table 1 parameters, the numbers from papers in which the Fe II template method was applied are preferentially quoted.

Spectra were available for analysis from the following objects: PHL 1092, 1H 0707-495, NGC 4051, Mrk 507 (kindly provided by J. Halpern), RE 1034+39 (kindly provided by D. Grupe), IRAS 13224-3809, IRAS 20181-2244 (kindly provided by L. Kay), IRAS 17020+4544 (Leighly et al. 1997b), Ark 564 (obtained from the HST archive), Mrk 142 and Kaz 163. The I Zw 1 template method was used to subtract the Fe II and the spectra were fitted using the LINER spectral fitting package (Pogge \& Owen 1993). Deblending of the $\mathrm{H} \beta$ line can contribute uncertainty to the derived parameters. Some fraction of $\mathrm{H} \beta$ originates in low ionization material from the narrow-line region (NLR); in NLS1s it is difficult to estimate how much arises from the NLR because the width of the $\mathrm{H} \beta$ is similar to that of the [O III] lines and therefore the narrow component cannot be easily recognized (e.g. Goncalves, Veron \& Veron-Cetty 1998). As it is beyond the scope of this paper and the signal to noise of many of the spectra to attempt a detailed deblending, I assume that the NLR contributes $\mathrm{H} \beta$ with $\frac{1}{10}$ the flux of $[\mathrm{O} \mathrm{III}] \lambda 5007$, and I subtract that from the $\mathrm{H} \beta$ profile before modeling. The assumption is based on the results obtained from narrow-line 
emission from Seyfert 2 and intermediate Seyfert galaxies (e.g. Koski 1978; Cohen 1983).

The $[\mathrm{O}$ III $] \lambda 5007$ line was modeled using one or two Gaussians. Two were required in the following objects; the second broader component was blueshifted with respect to the narrow component: RE 1034+39 (see also Mason, Puchnarewicz \& Jones 1996 and Goncalves, Veron \& Veron-Cetty 1998), NGC 4051, IRAS 17020+4544, Mrk 507 and IRAS 20181-2244 (see also Halpern \& Moran 1998). H $\beta$ and [O III] line fluxes were obtained by integrating the flux above the continuum, a procedure which has the advantage of being independent of the line model. The $\mathrm{H} \beta$ was finally fit with a Lorentzian profile, which provided a substantially better description in most cases than a Gaussian in the NLS1 spectra (also Goncalves, Veron \& Veron-Cetty 1998). In a few cases, subtraction of strong Fe II distorted the [O III $] \lambda 5007$ profile, making the FWHM unreliable (1H 0707-495). These results were merged with those from the literature to obtain the values in Table 1.

\section{Spectral Properties}

The spectral analysis is limited to the time averaged spectral properties of the sample. A preliminary investigation of spectral variability was discussed in Part 1. Detailed analysis of spectral variability has been presented already for Mrk 766 (Leighly et al. 1996) and NGC 4051 (Guainazzi et al. 1996), and will be discussed for Ark 564 in Leighly et al. in prep.

The spectra were first fitted with an absorbed power law with the absorption fixed at the Galactic value, and the ratio of the data to this model are shown for all the spectra in Figure 1. Significant deviations from the power law in nearly all of the spectra can be seen and the $\chi^{2}$ for this model are given in Table 2. An acceptable $\chi^{2}\left(\chi_{\nu}^{2}<1.3\right)$ was obtained from only 10 of the 24 spectral fits. Because of the wide range in fluxes represented by this sample, an acceptable fit may be obtained either when the model is adequate or when the statistics are poor. The shape of the residuals is generally concave upward and thus appears quite different than many of those from Seyfert galaxies with broad optical lines which tend to be modified by absorption (e.g. Reynolds 1997).

To investigate the origin of the residuals, initially two phenomenological models were tried. These consisted of a power law with a photon index $\Gamma$ and either a soft excess modeled as a black body with characteristic temperature $k T$ (the "soft excess" model) or an ionized absorber modeled as two absorption edges at 0.74 and $0.87 \mathrm{keV}$ in the galaxy rest frame (the "warm absorber" model). These models have the same number of degrees of freedom. The $\chi^{2}$ for these preliminary models are given in Table 2. The fit was improved substantially for 20 of the 24 spectra; only spectral fits to I Zw 1 and Kaz 163 were not improved at all, and the fit to IRAS 20181-2244 was improved only marginally. (Note that the following criteria were used to evaluate the spectral fits: the improvement was significant $(P<0.01)$ when $\Delta \chi^{2}>6.34,9.21$ and 11.3 for 1,2 and 3 additional parameters respectively; the improvement was marginal $(P<0.05)$ when $\Delta \chi^{2}>3.84$, 
5.99, and 7.82 for 1,2 and 3 additional parameters respectively.) Nineteen of the spectra (18 objects) are modeled substantially better by the soft excess model $\left(\Delta \chi^{2}=10\right.$, corresponding to a likelihood ratio of 140, e.g. Mushotzky 1982), and the fit was acceptable $\left(\chi^{2}<1.3\right)$ for 18 of these (all but 1H 0707-495). Three objects (IRAS 13349+2438, IRAS 17020+4544, Mrk 507) were fit substantially better using the warm absorber model, and IRAS 20181-2244 was fit marginally better $\left(\Delta \chi^{2}=6\right)$.

Some ambiguity remains after this preliminary modeling, as it is possible that the two-edge model may not be able to adequately describe the warm absorber. Therefore, a third preliminary model was applied, consisting of a power law, Galactic absorption and the absori model available in $X S P E C$; the resulting $\chi^{2}$ are listed in Table 2. The absori model describes the warm absorber and is characterized chiefly by two parameters: the column density $N_{w}$ and the ionization parameter $\xi$. The temperature of the ionized plasma is also an adjustable parameter; however, because the spectral fitting results are not very sensitive to this parameter, the temperature was fixed at either $3 \times 10^{4} \mathrm{~K}$ or $1 \times 10^{6} \mathrm{~K}$, and the lower value of $\chi^{2}$ is quoted. Note that the absori model does not include resonance line absorption. In 18 spectra from 17 objects, the $\chi^{2}$ for the absori model is more than 10 less than the $\chi^{2}$ for the two-edge model, indicating a likelihood ratio $>140$. In many of these objects, detailed analysis indicates the presence of a warm absorber, as discussed below. Alternatively, the ionization parameter becomes very low and neutral absorption is modeled (Mrk 507; IRAS 20181-2244). However, for the others, the ionization parameter becomes pegged at the maximum value of $\xi=5000$, where the model is not considered to be very accurate (P. Magdziarz 1997, P. comm.). When this is the case, the absori model appears to be trying to model the continuum. However, a soft excess models the continuum better. In nearly all cases, the difference in $\chi^{2}$ between the soft excess and absori models is larger than 10, indicating a likelihood ratio of $>140$. It is less than 10 only for Mrk 142; this is likely to be the fault of poor statistics, as the source is relatively faint and the exposure for the SIS0 is only 19 ks. Further evidence that the complexity of the spectrum is better described by a soft excess model is given by the lower panels in Figure 1. These show the ratio of the data to a power law (plus iron line as necessary) model fit above 2-3 keV, after the iron line component is removed from the model. The signature of absorption should be characterized by a deficit or localized features upon the continuum; in contrast, in many of the objects, a broad continuum excess is seen.

These preliminary results show that a soft excess is generally present in narrow-line Seyfert 1 galaxy $A S C A$ spectra. This situation contrasts with that from Seyfert galaxies with broad optical emission lines; Reynolds (1997) finds that the warm absorber fitted most of the spectra adequately, and a soft excess was required in only 3 cases.

These simple models do not adequately model all of the spectral residuals; evidence is also present for iron $\mathrm{K} \alpha$ lines and additional absorption. I follow Reynolds (1997) and proceed to fit with a phenomenological model with the following components, where all parameter values are obtained in the rest frame: 
- a power-law with photon index $\Gamma$ which models the high energy continuum;

- a soft excess modeled by a black body with temperature $k T$, which provides a generic model of the soft excess;

- two absorption edges with energies 0.74 and $0.87 \mathrm{keV}$ in the rest frame of the galaxy, which represent absorption by O VII and O VIII in ionized gas;

- Galactic absorption as listed in Table 3;

- intrinsic neutral absorption in the rest frame of the galaxy; in some cases, information from nonsimultaneous ROSAT spectra is used to constrain this parameter.

- an iron $K \alpha$ line, represented by a Gaussian with central energy $E$ and width $\sigma$.

The results are listed in Tables 3a, b, and c. Note that the values of the parameters and their errors $\left(\chi^{2}=2.71\right.$, that is, $90 \%$ for one parameter of interest) are listed only when there is a significant or marginal detection of that component. For the absorption edges and iron lines, upper limits are also listed.

Because the two-edge model may not adequately model the warm absorber in all cases, a complementary set of fits using the absori model was performed, and the results are listed in Table 4. These results are discussed in Section 3.3.

After applying this simple phenomenological model uniformly to the spectra, evidence for further complexity was discovered in 5 objects. There is additional evidence for an edge-like structure near $1 \mathrm{keV}$ in $1 \mathrm{H} 0707-495$, IRAS $13224-3809$ and PG $1404+226$. These features were discussed extensively in Leighly et al. 1997a; these features were modeled as edges here and will not be discussed. An additional soft X-ray emission line feature is seen around $1 \mathrm{keV}$ in PG 1244+026 and Ark 564. These features will be discussed in Section 3.4.

\subsection{The Photon Index in NLS1s}

The photon index from narrow-line Seyfert 1 galaxies appears to be on average steeper than that from Seyfert galaxies with broad optical lines. This result was first reported by Brandt, Mathur \& Elvis (1996), and it is confirmed by the spectra in this sample. Histograms of the photon indices for the NLS1s and for broad-line Seyfert 1 galaxies taken from Reynolds (1997) are plotted in Figure 2. The broad-line radio galaxies and 3C 273 are excluded; radio-loud objects often have flatter spectra than radio-quiet objects, possibly as the result of a flat component from the jet. Note that all of our narrow-line Seyfert 1 galaxies are radio quiet except PKS 0558-504 (Remillard et al. 1986). Also note that with or without the radio-loud objects, the distributions of the $2-10 \mathrm{keV}$ luminosities of the broad and narrow-line AGN are consistent as determined by a two-sample KS test. Figure 2 shows clearly that the photon indices are consistently steeper for 
the NLS1s. To test whether the photon indicies could have been drawn from the same parent population, the statistical package $A S U R V$ Rev. 1.2 (Isobe \& Feigelson 1990) which implements methods presented in Feigelson \& Nelson (1985) was used. A variety of tests are available in this package which make different assumptions about the underlying distributions. The differences were found to be highly significant $(P<0.0001)$ regardless of the assumed distribution.

The histogram shows an apparent overlap and spread in the values for both types of objects; however, it is difficult to determine whether this is significant from the histogram alone since the uncertainties in the photon indices are not taken into account. Therefore, the maximum likelihood method was used to determine jointly the best estimate of the mean and dispersion of both sets of indices (Figure 2; e.g. Maccacaro et al. 1988). These were $2.19 \pm 0.10$ and $0.30_{-0.06}^{+0.07}$ for the NLS1s and $1.78 \pm 0.11$ and $0.29_{-0.07}^{+0.09}$ for the broad line objects (errors are $68 \%$ for two parameters of interest). In both cases the dispersions are significantly greater than zero (99\% confidence $>0.19$ and 0.17 for the narrow and broad-line objects respectively; Figure 2). A significant spread in Seyfert galaxy X-ray 2-10 keV photon indices has been recognized previously by several investigators (e.g. Turner \& Pounds 1989; Nandra \& Pounds 1994). The contours representing the mean and dispersion exclude each other at greater than $90 \%$ significance. The validity of the maximum likelihood test depends on the assumption that the photon indices are normally distributed. This assumption is justified if the distributions are symmetric; however, the distribution of the broad-line objects appears to be significantly skewed $(S=-0.82 \pm 0.50)$. There is an interesting suggestion that high values are preferred for both types of objects, and the distributions tail toward the lower values.

\subsection{The Soft Excess in NLS1s}

A soft excess component is required to model 18 of the 24 spectra (17 of 23 objects). The most striking difference is in the degree of prominence of the soft excess in the spectra. This can be seen in the lower panels of Figure 1, which show the ratio of the data to a power law plus iron line (as necessary) model above $2 \mathrm{keV}$, and extrapolated to lower energies. This figure shows that the soft excess component in NLS1s stretches well into the ASCA band pass and that it can dominate the spectrum up to at least $1 \mathrm{keV}$. This situation contrasts with that from broad-line Seyfert galaxies; in that case, the soft excess is seen in only in the lowest channels in the $A S C A$ band, if at all (e.g. Guainazzi et al. 1994).

The measured temperatures of the soft excess component in the galaxy rest frame when modeled by a black body range from 0.1 to $0.25 \mathrm{keV}$. The maximum likelihood method gives an average of $0.15 \pm 0.05 \mathrm{keV}$ and dispersion of $0.12_{-0.05}^{+0.09} \mathrm{keV}$ (uncertainties are $68 \%$ confidence). The dispersion is significantly greater than zero at $>99 \%$ confidence; however, it must be kept in mind that there may be some model dependence in the temperatures. The measured temperature is likely to be correlated with the energy of the lowest spectral channel, which is not the same for all of the spectra. Observations from later in the mission generally had a higher event threshold 
than observations from early in the mission, and some observations were performed with a level discriminator. Also, the objects represent a range of redshifts. Furthermore, application of warm absorber edges and additional absorption can alter the measured temperature. Finally, physical models for the soft excess (e.g. an accretion disk spectrum) are likely to be characterized by a range of temperatures, although the inner edge temperature may be well defined.

To study the variation in strength of the soft excess, I borrowed the hardness ratio plot technique used in the study of Galactic X-ray sources. Figure 3 shows the ratio from the summed SIS0 and SIS1 spectra of the medium (1.0-2.0 keV) to soft (0.6-0.9 keV) energy bands on the $\mathrm{x}$ axis, and the ratio of the medium to the hard $(2.0-6.0 \mathrm{keV})$ on the y axis. There are two problems with applying this method directly. The first is that the Galactic column densities differ among the sample enough to seriously affect the flux in the soft band. Therefore, before accumulating the ratios, the spectra were corrected channel by channel for Galactic absorption using the analytic formulation from Morrison \& McCammon (1983). The second problem is that the redshifts of the sources differ significantly. This can be corrected using the ASCA SIS0 effective area curve; however, the correction becomes increasingly less accurate as the redshift increases as large changes in the effective area become shifted from one band to the other. The accuracy of this method is checked by simulating the spectra using the best fit models shifted to zero redshift. The result is that the correction gives acceptable results for all but the two highest redshift objects (PHL 1092 and RX J0493-45, with redshifts 0.396 and 0.224 respectively); therefore they are not included in the top panel of Figure 3. Finally, the hardness ratios are made from the luminosities predicted by the best fit models with the Galactic $N_{H}$ removed. These are shown in the bottom panel of Figure 3. The advantage here is that no $N_{H}$ or redshift correction is necessary; the disadvantage of this is that the luminosities are model dependent. Thus the top and bottom panels are complementary.

In Figure 3, the $\mathrm{x}$ axis, being the ratio of the medium to soft X-rays, displays the strength of the soft excess, where objects with strong soft excesses are on the left side of the plot and correspond to lower values of the ratio. Henceforth, this ratio will be referred to as the "color ratio". Six points marked by stars have very strong soft excesses: PHL 1092, RX J0439-45, 1H 0707-495, RE 1034+39, IRAS 13224-3809 and PG 1404+226. This group will be collectively referred to a "strong soft-excess objects". In the middle of the plot marked by circles are all the other objects with detectable soft excesses; note that NGC 4051 (2 observations) and Mrk 766 are separately marked with open circles. These will be referred to as "weak soft-excess objects". Note that although the strength or contrast of the soft excess is weak in these objects, the soft excess is required with high confidence in the spectral fit. On the left side of the plot marked by triangles are objects with no detected soft excess: I Zw 1, IRAS 13349+2438, IRAS 17020+4544, and Kaz 163. Note that absorption may be hiding a weak soft excess in some of these objects. Finally, the more heavily absorbed objects Mrk 507 and IRAS 20181-2244 are off the plot toward the right.

The $\mathrm{y}$ axis on this graph, displaying the ratio of the $1.0-2.0 \mathrm{keV}$ medium band to the 2.0-6.0 keV hard band, illustrates the steepness of the hard X-ray spectrum or power law, where 
large values correspond to steeper spectra. This assertion was verified by comparing the ratios with the measured photon indices. This graph shows that the strong and weak soft excess objects have almost the same distribution of photon indices. This means that the large range of soft excess strengths cannot be attributed to differences in the slope of the power law, but rather must be due to differences in the relative normalizations of the soft excess component and the power law.

The color ratio, as defined above, provides a measure of the strength of the soft excess. However, since only the effect of the Galactic absorption has been removed, the color ratio is also sensitive to intrinsic absorption, either ionized or neutral. Two other complementary parameters to measure the strength of the soft excess can be defined. These have the advantage that they should not be affected by the presence of intrinsic absorption; the disadvantage is that they are model dependent. The first, called the "black body parameter" is defined as the model flux color ratio between the black body component and the power law at $0.45 \mathrm{keV}$ plus the measured temperature of the black body in the rest frame $[(F(p l)-F(b b)) /(F(p l)+F(b b))]$. This parameter describes the prominence of the black body over the power law and it should be approximately directly proportional to the color ratio in the case where there is no intrinsic absorption. The values of the black body parameter are listed in Table 3. Because this parameter potentially depends most sensitively on the power law index, the uncertainties were evaluated by fitting the spectra with the power law index fixed at its $90 \%$ error limits. Values near -1 and 1 imply very strong and very weak soft excesses, respectively. The black body parameters range from -0.72 for IRAS 13224-3809 to 0.77 for PKS 0558-508 (not counting objects with no detectable soft excess, for which the black body parameter equals 1 ). These values imply that the black body component is a factor of $\sim 50$ stronger in IRAS 13224-3809 than in PKS 0558-508.

The final parameter is $\alpha_{x x}$. Defined as the slope between 0.7 and $4 \mathrm{keV}$ in the rest frame, it measures the overall steepness of the continuum spectrum. This slope is computed from the modeled continuum and therefore should also be independent of intrinsic absorption; however, it is model dependent. This parameter indirectly measures the strength of the soft excess: when there is no soft excess or it is weak, $\alpha_{x x}$ is close to the photon index; when the soft excess is strong, $\alpha_{x x}$ is much larger than the photon index.

\subsection{Neutral and Ionized Absorption in NLS1s}

Evidence for an ionized "warm" absorber was found in more than half of a sample of $A S C A$ spectra from bright, predominantly broad-line Seyfert galaxies (Reynolds 1997; also George et al. 1998), a result which supports the idea that absorption by highly ionized material may be ubiquitous in Seyfert galaxies. In contrast, significant detections of a warm absorber as modeled

by two absorption edges with fixed energies were found in only 6 spectra from 5 of the 23 objects; marginal detections were obtained from 5 others. It is conceivable that this is a consequence of the low flux level of our objects; however, there is also the impression that when a warm absorber is present, it has a lower optical depth, especially in the higher ionization, O VIII edge. The 
detections and upper limits are plotted in Figure 4, along with the results from broad-line AGN given in Reynolds (1997). Note that several of Reynold's detections were changed to upper limits for consistency with the detection criteria adopted here. This figure shows that many of the O VIII upper limits from the NLS1s are below the detections from the broad-line objects.

The possibility of a difference in warm absorber parameters for the broad and narrow-line objects was investigated by running statistical tests on the measured edge depths and upper limits from the sample of NLS1s presented here and from the comparison sample of broad-line AGNs from Reynolds (1997). Note that because the warm absorber is not detected in all objects, the upper limits should be taken into account properly using survival analysis statistical methods (e.g. Feigelson \& Nelson 1985); and here they were accounted for using the Kaplan-Meier estimator as implemented in the $A S U R V$ package. The resulting average optical depths for O VII and O VIII are $0.19 \pm 0.04$ and $0.053 \pm 0.020$ respectively for the NLS1s, and $0.29 \pm 0.07$ and $0.18 \pm 0.06$ for 21 broad-line objects. Note that in the algorithm when the minimum optical depth is an upper limit, it must be changed to a detection; this occurred in both data sets so the mean could be biased. Nevertheless, these results suggest that the O VII optical depths are consistent between broad and narrow-line objects, while the O VIII optical depths are smaller for the narrow-line objects compared with the broad. To test this further, ASURV two-sample tests which assume a variety of different intrinsic distributions were run. No difference between O VII optical depths was found (probability of a significant difference of 29-43\%; a range is found for the different assumed intrinsic distributions), but a difference between the O VIII optical depths at 97-98\% confidence was found. Reynolds (1997) found some evidence for a difference between high and low luminosity objects that could possibly be attributed to different behavior of radio-loud objects. If the radio-loud objects are excluded from the broad-line sample, there is still no difference in $\tau_{O V I I}$, but the significance of the difference in $\tau_{O V I I I}$ becomes $>99 \%$. Finally, warm absorber in the broad-line Seyfert 1 galaxy NGC 3783 has a very high optical depth; it is possible that this galaxy skews the results. If it is excluded, no difference in $\tau_{O V I I}$ is obtained, but there is still a significant difference in $\tau_{O}$ VIII of $>98 \%$.

The fact that the average O VIII optical depth is lower, that many of the upper limits on the NLS1 $\tau_{O \text { VIII }}$ are smaller than the broad-line object detections, and that there is an apparently robust significant difference in the distribution of $\tau_{O}$ VIII suggests that the ionization state in NLS1s is lower on average than in broad-line Seyfert galaxies. A better way to test this supposition would be to compare ratios of edge optical depths; however, it is difficult to determine a sensible way to derive the ratio of the edges when both values are upper limits. However, the joint distributions of $\tau_{O V I I}$ and $\tau_{O V I I I}$ can be compared using a two sample test for multivariate data containing upper limits (Makuch, Escobar \& Merrill 1991). Testing against the hypothesis that the joint $\tau_{O V I I}$ and $\tau_{O V I I I}$ distributions are equal, I find mixed results. If the generalized Gehan distribution is appropriate, the warm absorber properties from the NLS1s are not significantly different from those of the broad-line Seyferts at 84-91\% confidence. However, if the logrank distribution is appropriate, indications are that the difference may be significant (95-98\%). Since 
it is not clear which distribution is appropriate, I conservatively conclude that there is a suggestion rather than a discovery that the distribution of warm absorber properties as a whole is different in NLS1s than broad-line AGN. However, the difference in distribution of the O VIII optical depths remains significant.

All of the spectra were also fit with warm absorber modeled using the absori model in $X S P E C$, and the results are listed in Table 4. A warm absorber was detected using this model in all of the objects in which one was detected using the two-edge model. A warm absorber was detected with low significance in several other objects as well (marginal detections in PG 1244+026, IRAS $13224-3809$, Kaz 163; $\Delta \chi^{2}=10$ in Ark 564).

Additional neutral absorption in the rest frame of the galaxy was detected in 4 objects: I Zw 1, IRAS 17020+4544, Mrk 507 and IRAS 20181-2244. I Zw 1 is a moderately bright object, and thus since the detection of absorption is not very significant, the necessity of this component is somewhat doubtful. Furthermore there is marginal detection of a warm absorber and the two components are probably coupled in the spectral fitting. Significant absorption is indicated in the ROSAT spectrum, but at a much lower level $\left(N_{H}=2.3 \times 10^{20} \mathrm{~cm}^{-2}\right)$. Further broad band observations are are necessary to determine whether this is significant or not. The absorption in Mrk 507 has been discussed by Iwasawa, Brandt \& Fabian (1998); it is likely to be the reason that the ROSAT photon index is so flat in this object. Significant neutral absorption as well as ionized absorption was reported in IRAS 17020+4544, and has been linked to the high optical polarization in this object (Leighly et al. 1997b). The absorption in IRAS 20181-2244 has been discussed by Halpern \& Moran (1998), and is probably the reason that this object was so faint in the ROSAT All Sky survey.

It is important to note that soft excesses were not detected in several of the objects with warm or neutral absorbers. This may be because weak soft excesses cannot be distinguished when there is also absorption. Thus, soft excesses may be even more prevalent in NLS1s than is indicated here. It is possible that the presence of a soft excess can also make detection of a warm absorber more difficult, since the absorption structure may be easier to see when the continuum is a power law. However, the moderate energy resolution provided by $A S C A$ should be adequate to allow us to detect features from the warm absorber even if they fall upon a multicomponent continuum.

Leighly et al. 1997b discovered that highly polarized Seyfert 1 galaxies almost always had a warm absorber. This result supports the hypothesis of dusty warm absorbers (Brandt, Fabian \& Pounds 1996; Reynolds et al. 1997); that is, dust which reddens and polarizes the optical emission is coincident with ionized gas. Several of the NLS1s discussed here have high optical polarization, including I Zw 1 (Smith et al. 1997), NGC 4051, Mrk 766 (Goodrich 1989), IRAS 13349+2438 (Wills et al. 1992), IRAS 17020+4544 (Leighly et al. 1997b), Mrk 507 (Goodrich 1989) and IRAS 20181-2244 (Kay et al. 1999). All of these objects show evidence for either neutral or ionized absorption. The following objects have weak or no polarization: Mrk 335, Mrk 142, PG 1211+143, PG 1244+026, PG 1404+226, Mrk 478 (Berriman et al. 1990), IRAS 13224-3809 
(Kay et al. 1999), and Ark 564 (Goodrich 1989). There is either no or only marginal evidence for a warm absorber in these, except for Mrk 478. Polarization properties of the following objects have not yet been reported: Ton S180, PHL 1092, RX J0439-45, NAB 0205+024, PKS 0558-504, 1H 0707-495 and Kaz 163. Thus the NLS1s appear to show the same trend of absorption and polarization as we found for Seyfert galaxies in general (Leighly et al. 1997b).

\subsection{Soft X-ray Features in NLS1s}

Features resembling absorption edges near $1 \mathrm{keV}$ were found in the $A S C A$ spectra from the three NLS1s: 1H 0707-495, IRAS 13224-3809 and PG 1404+226. This work is discussed in detail in Leighly et al. 1997a. These features could be fit either by a one or two absorption edges or two or three unresolved absorption lines. Oxygen offers the primary opacity in photoionized gas; however, the energies of the absorption features are much too high to be attributed to oxygen absorption. If interpreted as absorption due to oxygen, the energies of these features imply a high blueshift of the absorbing material: $0.2-0.3 \mathrm{c}$ for the edge model and near $0.57 \mathrm{c}$ for the line model. It is difficult to accelerate ionized gas to such high velocities; however, the notable similarities between NLS1s and low-ionization broad absorption line quasars (BALQSOs) prompt us to take this hypothesis seriously. Both NLS1s and BALQSOs show strong or extreme Fe II and weak [O III] emission; many NLS1s and low-ionization BALQSOs have red optical spectra and strong infrared emission; finally, both classes are predominantly radio-quiet. See Leighly et al. 1997a for more details. An alternative interpretation in terms of a highly ionized warm absorber dominated by resonance absorption lines dominated by Fe L has been proposed by Nicastro, Fiore \& Matt 1999. For this model, it is probably relevant that these absorption features near $1 \mathrm{keV}$ are found exclusively in the objects with the strongest soft excesses in the sample.

The spectra of two other NLS1s, PG $1244+226$ and Ark 564 revealed excess emission near $1 \mathrm{keV}$ (Figure 1). The presence of this feature in PG $1244+226$ has been discussed by Fiore et al. 1998. The residual can be most simply modeled as an unresolved Gaussian emission line. The features are clearly significant in addition to a weak soft excess component. This is demonstrated by energy versus flux $\chi^{2}$ contours shown in Figure 5, where the narrow width has been fixed at the best fit value to better constrain the fit; when the line width is left free, it becomes confused with the soft excess when at large fluxes and widths.

Fiore et al. 1998 discuss possible origins of this line. Emission in an optically thin thermal plasma is highly unlikely because the large luminosity requires a large amount of gas, and the rapid variability implies a small emission region. The combination of these two constraints implies the gas must be optically thick, in contrast with the assumption that the gas is optically thin. Rather, they support the hypothesis that this feature may arise from reflection in a photoionized accretion

disk. This hypothesis is supported by the idea that NLS1s are accreting at a higher fraction of the Eddington rate, since under that condition, the accretion disk is expected to become ionized (e.g. Matt, Fabian \& Ross 1993a). The energy near $1 \mathrm{keV}$ supports interpretation as a blend of Ne IX 
and $\mathrm{Ne} \mathrm{X}$ at 0.92 and $1.02 \mathrm{keV}$ respectively, as well as Fe L lines at around $0.8 \mathrm{keV}$. The lack of a $\mathrm{O}$ VIII $\mathrm{K} \alpha$ line near $0.65 \mathrm{keV}$ is puzzling however as it is expected to be strong in photoionized plasmas (e.g. Netzer 1996).

Fiore et al. 1998 also note that in the Matt et al. (1993a) models, the disk ionization parameter $\xi$ depends on the accretion rate as $\dot{m}^{3}$. The sensitivity of this dependence means that while the accretion rate may be large in many NLS1s and therefore while the disk may be ionized in many NLS1s, observation of these features would not necessarily be expected in all of them. Note that the $\xi \propto \dot{m}^{3}$ dependence is based on several assumptions; two primary ones are that the fraction of accretion energy going into X-rays which illuminate the disk remains the same as the accretion rate increases and that the geometry also remains the same.

\subsection{The Iron Line in NLS1s}

An iron $\mathrm{K} \alpha$ line with energy consistent with emission from neutral or low-ionization-state iron is ubiquitous in $A S C A$ spectra from low-luminosity broad-line Seyfert 1 galaxies (e.g. Nandra et al. 1997a; Reynolds 1997). Frequently the line is found to be significantly broader than the instrument resolution (e.g. Mushotzky et al. 1995). The signal to noise is sufficient in at least one observation that the line profile can be examined; a clearly identified red wing provides the best evidence so far for emission from a relativistic accretion disk near a black hole (MCG-6-30-15; Tanaka et al. 1995). At higher luminosities, however, the iron line appears to be less frequent, and there is some indication that the characteristic ionization state of the iron is observably higher (Nandra et al. 1997b).

It is important to compare the iron line properties from narrow-line Seyfert 1 galaxies with those from broad-line Seyfert galaxies in order to place constraints on the geometry and importance and conditions of reprocessing. This is a difficult task using these data, however. The low flux combined with the steepness of the spectrum means that the signal to noise at the iron line is generally very poor. In fact, some of the sample were not significantly detected above 6.0 $\mathrm{keV}$. For this reason, no useful constraints could be obtained from the following: 1H 0707-495, PG 1404+226 and RE 1034-3809.

To examine the iron line in the remaining 20 objects, the spectra above $2 \mathrm{keV}$ were fit, rather than the whole band pass, so that potential broad band spectral curvature would minimally affect the results. Some evidence for an iron line was found in 13 of the 20 objects detected in the iron line energy range. An iron line was not statistically necessary in the models for PHL 1092, RX J0439-45, NAB 0205+024, Mrk 142, Mrk 507, Kaz 163 and IRAS 20181-2244. It is interesting to note that in PHL 1092 and IRAS 20181-2244 an iron line is found just below the detectability threshold (PHL 1092: $\Delta \chi^{2}=5.3$; IRAS 20181-2244: $\Delta \chi^{2}=5.6$ ) and in both cases the best fitting line energy is high, near $6.8-6.9 \mathrm{keV}$. An upper limit was obtained from the nondetections by fitting with an narrow Gaussian $(\sigma=0.05 \mathrm{keV})$. The rest frame energy 
was fixed at either the best fitting energy or at 6.4 and $6.7 \mathrm{keV}$ and the result from the better fit was reported. These results show that the upper limits on the equivalent widths for these 7 objects are commensurate with those from the detections, and the line fluxes are on the low end of the distribution. This implies that lines were not detected in these objects simply because the statistics are poor.

In 6 spectra from 6 objects the uncertainty on the width of the line excludes zero at $90 \%$ confidence for 1 parameter of interest. $\chi^{2}$ contours were constructed from fits in which the line energy, width and flux were allowed to vary and a large region of parameter space was explored. These show that for two parameters of interest, the iron line is broad at the $90 \%$ confidence level in all 6 objects: I Zw 1, Ton S180, NGC 4051, Mrk 478, IRAS 17020+4544 and Ark 564. Not surprisingly, those are among the brightest objects at high energies. Interestingly, the line was unresolved in Mrk 335 and Mrk 766, both bright objects. Evidence for both broad and narrow iron lines was found in the first observation of NGC 4051.

In 6 of the spectra, the best fit line energy and $90 \%$ confidence interval for one parameter of interest exclude neutral iron emission at $6.4 \mathrm{keV}$. However, note that the line detection is marginal or nearly marginal in 4 of these. $\chi^{2}$ contours show that, for two parameters of interest, the line energy excludes $6.4 \mathrm{keV}$ in only two of the objects ( $\mathrm{I} \mathrm{Zw} 1$ and Ton S180) at greater than 90\% confidence level when the line was allowed to be broad. The line energy in Ton S180 excludes $6.4 \mathrm{keV}$ at $99 \%$ confidence (Figure 6; see below). The line energy excludes $6.4 \mathrm{keV}$ when the line width was constrained to be narrow in three other objects: PKS 0558-504, PG 1244+026, and IRAS $13349+2438$.

The average equivalent width, evaluated using the Kaplan-Meier estimator in ASURV which takes into account upper limits, was computed using the results from the 20 objects in which the iron line properties could be investigated. The resulting average was $352 \pm 77 \mathrm{eV}$. This is consistent with the average of $362 \pm 56 \mathrm{eV}$ for radio-quiet broad-line objects from Reynolds (1997). The distributions of equivalent widths are not found to be significantly different using two sample tests implemented in $A S U R V$. There are some difficulties with a direct comparison, however. A difference in equivalent width should be expected based solely on the difference in photon index: the equivalent width of the line is expected to be $\sim 20 \%$ larger when the photon index is 1.9 compared with 2.3, assuming a face-on viewing angle, because there are relatively fewer photons above the iron K-edge in the steeper spectrum (George \& Fabian 1991). The equivalent widths of detected lines may be artificially large in the NLS1s because the statistics are poorer in those spectra (a Malmquist bias). The energies of some of the lines are consistent with emission from ionized iron and under these conditions the fluorescence yield can be either enhanced or depressed depending on the ionization state (e.g. Matt, Fabian \& Ross 1993b).

Since the equivalent width relative uncertainty is approximately the same as the line flux relative uncertainty (Yaqoob 1998), a maximum likelihood test on the 12 significant and marginal detections could be done. This revealed a significant dispersion on the equivalent widths at 
$90 \%$ confidence for two parameters of interest: the mean and dispersion are $270_{-110}^{+140} \mathrm{keV}$ and $190_{-110}^{+130} \mathrm{keV}$, respectively.

The inclination of the disk is also important. The five of the six objects in which a broad line was detected with $90 \%$ confidence were fit with a model representing a emission line emerging from a planar geometry near a Schwarzschild black hole (Fabian et al. 1989); note that the detection in Mrk 478 was too nearly marginal to provide any useful constraints). Poor statistics prevented many of the parameters from being constrained; therefore, the inner and outer radii were fixed at 6 and 1000 gravitational radii, respectively, and the emissivity parameter was fixed at its best fit value between -2 and -3 . The results shown in Figure 1 illustrate that useful constraints could only be obtained for NGC 4051. Since the inclination is determined by the presence of a blue wing, the steep spectrum, conspiring with the low flux, make the inclination especially difficult to constrain in $A S C A$ data from NLS1s.

Two objects present particularly interesting iron line behavior. I Zw 1 has a strong hard excess in its spectrum (Figure 7) which appears to be a very large equivalent width iron line. This result is interesting in light of the fact that I Zw 1 is the prototype narrow-line quasar with very strong optical Fe II emission (e.g. Phillips 1976). The iron emission in the UV is also very strong (Laor et al. 1997b). Thus it may not be surprising that the iron emission in X-rays is also strong, although if it is connected with the optical and UV results it may imply a nucleus-wide overabundance of iron, since the emission mechanisms for Fe II and iron K $\alpha$ are much different. Ton S180 has the clearest example of a broad, ionized iron line among the objects in our sample. Such a line was previously detected in the $S A X$ data on this object (Comastri et al. 1998). The $\chi^{2}$ contours of the energy versus the width are shown in Figure 6.

In summary, it appears that the equivalent width of iron line is the same in NLS1s as in broad-line objects; this would imply that the covering fraction of optically thick material is the same in both types of objects. This result is severely limited by the statistics of the spectra, however.

\subsection{Correlation Analysis}

This paper and Part 1 present the X-ray spectral and variability results as well as some optical emission line properties from a sample of narrow-line Seyfert 1 galaxies observed by $A S C A$. It is appropriate to search for correlations among these properties. Nonparametric correlation coefficients are more robust against outliers and more appropriate for these data which are not expected to be distributed normally. The Kendall's Tau statistic was used (e.g. Press et al. 1992), primarily because there is an implementation with reliable confidence estimates available in $A S U R V$. Tests for correlations were also done using the more familiar Spearman rank statistic, and the results were not substantially different.

Two sets of correlations were performed. One set included data from all 23 objects, and the 
other used data from a subsample consisting of only the 17 objects that possessed an observable soft excess. There were two observations of NGC 4051, and the data from the second observation was used for the correlations owing to the longer exposure and better statistics. The correlation matrix, listing the probabilities that the data are uncorrelated, is given in Table 5. Plots of some of the correlations are given in Figure 8. I refer to those correlations having probabilities of no correlation of $<0.06$ to be significant or suggestive, and those with probabilities of $<0.01$ to be strong. Significant and strong correlations are marked in Table 5 by open and solid circles respectively.

Correlations between the optical emission line properties, including $\mathrm{H} \beta \mathrm{FWHM}, \mathrm{H} \beta$ equivalent width, the ratio of Fe II to the $\mathrm{H} \beta$ emission and the ratio of [O III] to $\mathrm{H} \beta$ emission, were examined. Note that detection of a significant correlation between $\mathrm{H} \beta \mathrm{FWHM}$ and any parameter is somewhat surprising, considering that the sample of objects is selected on the basis of small $\mathrm{H} \beta \mathrm{FWHM}$ and therefore the dynamic range for this parameter is small. A strong correlation was found between the $\mathrm{H} \beta$ FWHM and the $\mathrm{H} \beta$ equivalent width. This correlation has been discussed by Goodrich (1989) and references therein, and indicates a connection between the kinematics of the broad-line region and the physical conditions within the emitting gas. This correlation was also found to be strong in a sample of optically selected NLS1s observed by ROSAT that was considered by Forster (1998). Correlations were also performed between the Fe II equivalent width, which can be approximated as the product of the $\mathrm{Fe} \mathrm{II} / \mathrm{H} \beta$ ratio and the $\mathrm{H} \beta$ equivalent width, assuming the continuum is approximately flat. An anticorrelation between Fe II equivalent width and $\mathrm{H} \beta$ FWHM has been previously reported by many investigators including Zheng \& O'Brien 1990, Puchnarewicz et al. 1992, Grupe et al. 1999a, and Boroson \& Green 1992. Interestingly, I find a positive correlation between these properties. Forster (1998) also finds this result; specifically, he finds that for low $\mathrm{H} \beta$ FWHM, there is a correlation with Fe II equivalent width which shifts to an anticorrelation for higher $\mathrm{H} \beta$ FWHM.

Correlations were sought between the $A S C A$ and $R O S A T$ photon indices and the optical line properties. A significant correlation was found between the $\mathrm{H} \beta$ FWHM and the $A S C A$ hard $\mathrm{X}$-ray photon index (Figure 8). There is also a strong correlation between $\mathrm{H} \beta$ equivalent width and the $A S C A$ photon index which is probably a consequence of the strong correlation between the $\mathrm{H} \beta$ parameters discussed above. These correlations are important because they imply a direct connection between the conditions in the central engine and the kinematics of the broad-line region. This result is also important because it challenges some models proposed for the production of the strong Fe II characteristic of NLS1s, as has been noted previously. The large Fe II to $\mathrm{H} \beta$ ratio is generally difficult to understand because both emission lines are predicted to be produced under the same conditions in photoionization models and those models generically underestimate Fe II (for a review, Joly 1993). It has been proposed that strong Fe II emission would be produced if the $\mathrm{X}$-ray spectrum is flat because hard X-ray photons can penetrate deeper into emission line clouds producing heating and leading to strong Fe II emission. However, an anticorrelation between $\mathrm{H} \beta$ FWHM and the X-ray photon index has always been found (Puchnarewicz et al. 1992, Boller, 
Brandt \& Fink 1996, Grupe 1996 and Grupe et al. 1999a, and Brandt, Mathur \& Elvis 1997, Wilkes, Elvis \& McHardy 1987). Most of these studies used soft X-ray spectral indices obtained from ROSAT or Einstein IPC. Therefore it is interesting that the correlation between $\mathrm{H} \beta \mathrm{FWHM}$ and the soft X-ray photon index measured using ROSAT is not significant. Conceivably this could be an effect of absorption which would tend to flatten the soft X-ray spectrum. However, the subsample of objects that have detectable soft excesses are minimally affected by absorption, and a significant correlation is not found within that sample either. Figure 8 shows that the ROSAT photon index is clustered around 3 for most of the sample except for the objects with the strongest soft excesses, and those with significant absorption.

No correlation is observed between the $A S C A$ and ROSAT photon indices, even for the relatively unabsorbed, soft excess subsample. Again, this is apparently because $\Gamma_{R O S A T}$ is clustered between 3 and 4 for a broad range of $A S C A$ photon indices. The lack of correlation between soft and hard photon indices is interesting because it appears to not support the most direct interpretation of the model proposed by Pounds, Done \& Osborne (1995) for NLS1s. They proposed that the steep hard X-ray photon index is produced when the Comptonizing electron cloud is cooled by strong soft excess emission, in analogy with Galactic black hole candidates in the high state (e.g. Nowak 1995). The steep ROSAT spectrum implies a strong soft excess (see below); therefore, one might expect that the abundance of soft photons available when the soft excess is strong would predict that the strongest soft excesses would be associated with the steepest hard X-ray spectra. However, the soft photons necessary to cool the electrons may be emitted in the unobservable EUV and therefore a correlation between these indices is not required for the model to be viable.

Correlations between the overall shape of the $A S C A$ spectra and the strength of the soft excess with other parameters may be important to examine. Three complementary parameters as defined in Section 3.2 (the color ratio parameter, the black body parameter and $\alpha_{x x}$ ) were used to to describe the overall shape of the $A S C A$ spectra. These parameters are very strongly correlated, as expected. A strong correlation was found between $\alpha_{x x}$ and the $A S C A$ photon index. This correlation is expected since these parameters are not independent: $\alpha_{x x}=\Gamma_{A S C A}-1$ when there is no soft excess. There is no correlation between the color ratio and the $A S C A$ photon index; this appears to be because the color ratio includes intrinsic absorption, which would not be expected to be correlated with intrinsic continuum parameters. The correlation is weak between the black body parameter and the $A S C A$ photon index; again, this supports the idea that the hard X-ray photon index is independent of the strength of the soft excess in the $A S C A$ band. The color ratio and $\alpha_{x x}$ are both correlated with the ROSAT photon index, a result that may originate in two effects. The color ratio correlation appears to be dominated by absorption; that is, objects with high color ratio and low ROSAT photon index are also the ones that were found to have warm or neutral absorption in their $A S C A$ spectra. On the other hand, $\alpha_{x x}$ should be largely independent of absorption and thus this correlation is due to the fact that objects with steeper ROSAT spectra have overall steeper $A S C A$ spectra. Figure 8 shows that most of the strong soft excess objects are 
located in the upper right corner of the $\alpha_{x x}-\Gamma_{R O S A T}$ plot.

Correlations between the variability parameters and other parameters are also important; however, they must be examined carefully. Figure 3 in Part 1 shows that the excess variance appears correlated with the luminosity. However, the measured correlation is weak, a result which may be caused by the large scatter. Recall from Part 1 that the skew parameter is defined as the number of sigma from the predictions of a very specific model; it is therefore more reliable for high values rather than low values which would be dominated by fluctuations.

A most interesting result is the evidence for correlations between the variability parameters and the overall shape of the ASCA spectra. Consistently, more variable objects have steeper spectra. The parameter $\alpha_{x x}$ was chosen to be most representative of the intrinsic steepness and is plotted versus excess variance and skew in Figure 8. This figure illustrates that a primary reason for this correlation is that the objects with strong soft excesses are more variable than the ones with weak soft excesses. To investigate this further, the distribution of properties shown in Figure 8 for the strong (6 objects) and weak (9 objects) soft excess objects were investigated using several two-sample tests implemented in $A S U R V$. The emission line parameters, including $\mathrm{H} \beta$ FWHM, $\mathrm{H} \beta$ equivalent width, Fe II equivalent width and ratio of $\mathrm{Fe}$ II to $\mathrm{H} \beta$, are distributed equally among the strong and weak soft excess objects. This is important because it suggests that the correlation between the overall shape of the $A S C A$ spectra and the variability properties may not be associated with the Boroson \& Green (1992) Eigenvector 1. There is no difference in distributions of the $A S C A$ photon index or $2-10 \mathrm{keV}$ luminosity either. There are only strong differences in the distributions of the variability parameters, as indicated by the plots.

Correlations between the iron line equivalent width and all of the other parameters listed in Table 5 were also considered. Only one significant correlation was found: an anticorrelation between color ratio and the equivalent width for the soft excess objects which was significant at 0.058 .

Several other significant correlations were found. The color ratio is strongly correlated with the optical depth of O VII. This is expected, based on the sensitivity of the color ratio to absorption. Similarly, the ROSAT photon index is correlated with $\tau_{O V I I}$, a further indication that the measured ROSAT photon index is influenced by unmodeled absorption. The temperature of the black body model for the soft excess is not significantly correlated with any parameter. It is weakly anticorrelated with the black body parameter, a result that may reflect model dependence. There is an intriguing correlation between the Fe II to $\mathrm{H} \beta$ ratio and the variability parameters for the soft excess objects. This appears to be due to the fact that some of the strong soft excess objects have high Fe II to $\mathrm{H} \beta$ ratios.

In summary, the most interesting correlations are as follows: a significant correlation between $A S C A$ photon index and $\mathrm{H} \beta \mathrm{FWHM}$ was found; however, no correlation between $R O S A T$ slope and $\mathrm{H} \beta$ FWHM was found. The strength of the soft excess was not correlated with the hard X-ray photon index, as would be naively expected from Compton cooling models, but is correlated with 
the variability parameters. This is especially interesting because the latter correlation does not appear to be associated with the Boroson \& Green (1992) Eigenvector 1.

\section{Discussion}

The discussion is organized as follows. First, the constraints imposed by the results on current models for X-ray emission and absorption are discussed in a general way. Then, the consistency of the results on general models based on the orientation and accretion rate are discussed.

\subsection{The Photon Index}

The photon indices in the sample of NLS1s presented here was found to be systematically steeper than those in the broad-line Seyfert 1 galaxies analyzed by Reynolds (1997). A maximum likelihood analysis showed that the difference is significant at $90 \%$ confidence, and there is significant dispersion among the photon indices of both types of objects. Brandt, Mathur \& Elvis (1997) previously reported this result. The advantage of the analysis presented here is that it is uniform, whereas Brandt et al. took their results from the literature and therefore the continuum model or fitting range could be different from object to object. This is conceptually important, because the soft excesses in some objects are strong enough and extend to high enough energy that they could modify the spectrum above $2 \mathrm{keV}$ and skew the photon index obtained from a 2-10 keV fit. In practice, however, the results are probably within the errors obtained from both approaches.

\subsubsection{Thermal Comptonization}

Pounds, Done \& Osborne (1995) proposed that, in analogy with the soft state of Galactic black hole candidates, the steep hard X-ray photon index in the NLS1 RE $1034+39$ can be understood in terms of a thermal Comptonization model. The energetic electrons are cooled by the copious soft photons from the soft excess, and the resulting cooler temperature leads to a steeper spectrum. This scenario was also adopted by Brandt, Mathur \& Elvis (1997) to explain the anticorrelation between $\mathrm{H} \beta \mathrm{FWHM}$ and $A S C A$ photon index.

However, the simplest thermal Comptonization models (e.g. Rybicki \& Lightman 1979), will not work for AGN, because they predict a far more sensitive photon index dependence on the electron temperature and optical depth than is observed (e.g. Haardt \& Maraschi 1991). Feedback between the soft photon source (cold phase) and the energetic electrons (hot phase) is required to stabilize the models and produce photon indices fairly narrowly distributed around a single value (e.g. Figure 2). Feedback models have been developed by Haardt \& Maraschi 1991, 
1993, Ghisellini \& Haardt 1994, and Pietrini \& Krolik 1994. Generally, the feedback consists of reprocessing of radiation from the hot phase by the cool phase, which increases the luminosity of the cool phase, as well as pair creation in the hot phase which alters the optical depth. Pietrini \& Krolik (1994) find that the plasma parameters can be described by two scaling laws which are weak functions of the ratio of hard and soft luminosity: $\theta \tau_{T} \simeq 0.1\left(l_{h} / l_{s}\right)^{1 / 4}$ and $\alpha \approx 1.6\left(l_{s} / l_{h}\right)^{1 / 4}$, where $\theta=k T / m_{e} c^{2}$ is the plasma thermal energy in units of electron rest energy, $\tau_{T}$ is the scattering optical depth, $\alpha$ is the power law energy index and the compactness is $l=\left(\sigma_{T} / m_{e} c^{3}\right)(L / R)$. A difficulty with these feedback models is that if the hot phase completely covers the cool phase, and if all of the dissipation occurs in the hot phase, the predicted photon index will be far steeper than observed in broad-line Seyfert 1 galaxies. This problem has been addressed by proposing that covering is not complete; instead, the hot phase is confined to isolated hot spots (Haardt, Maraschi \& Ghisellini 1994; Stern et al. 1995). If the spots hug the surface of the accretion disk, then the hot phase will intercept few photons from the cool phase, and the resulting photon index will be flat. If instead the hot spot is tall and thin, many soft photons will be intercepted, and the resulting photon index will be steeper.

The scaling law can be used to estimate the difference in $l_{s} / l_{h}$ ratio in NLS1s and broad-line Seyfert 1 galaxies. Assuming that the intrinsic indices are $\sim 2.4$ and $\sim 2.0$ for NLS1s and broad-line Seyfert 1 galaxies, respectively, means that $l_{s} / l_{h}$ should be a factor of 3.8 larger in NLS1s. Pietrini \& Krolik (1994) point out that $l_{s}$ can be interpreted as a combination of intrinsic soft X-ray luminosity and of geometry (efficiency of reprocessing); therefore, if the geometry and therefore reprocessing is the same in NLS1s and broad-line Seyfert 1s, then the intrinsic soft X-ray luminosity must be higher to explain the difference in index. However, the amount of reprocessing and therefore geometry is not well constrained in NLS1s. The iron line equivalent width potentially gives some information about the amount of reprocessing. In the sample of NLS1s presented here, the iron emission line has approximately the same equivalent width as the lines in broad-line Seyfert 1s, overall, arguing that the covering fraction of reprocessing material in NLS1s is about the same as in broad-line objects. However, the iron line measurements have very large uncertainties. Also, there is evidence for an ionized iron line in a few objects; ionization of iron can result in either enhanced or reduced equivalent widths compared with neutral iron, depending on its ionization state (e.g. Matt, Fabian \& Ross 1993b). Because there is very little information above $10 \mathrm{keV}$ from NLS1s, the amount of Compton reflection cannot be determined. NGC 4051 and Mrk 335, the only NLS1s considered by Nandra \& Pounds (1994), both have evidence for a reflection component in their Ginga spectra.

Spectral variability provides a potential diagnostic for this model. Assuming the geometry and plasma parameters remain the same, because of the shallow dependence of $\alpha$ on $l_{s} / l_{h}$, little spectral variability is expected for moderate changes in flux. It is also worth noting that these thermal comptonization models cannot explain the spectral variability exhibited by Mrk 766 as a change in $l_{s} / l_{h}$ (Leighly et al. 1996). In this case, a change in geometry would be required also.

A simple interpretation of the Pietrini \& Krolik (1994) scaling law indicates that there should 
be a correlation between the strength of the soft excess, if it represents $l_{s}$, and the hard X-ray photon index, if it represents $l_{s} / l_{h}$. The results presented here do not support this, as there is no significant correlation between the hard X-ray photon index and any of the soft excess indicators (except $\alpha_{x x}$ which is not independent of the photon index). Figure 8 shows that strong soft excess objects are distributed more or less equally above and below the maximum likelihood average photon index of 2.19, and there are several weak soft excess objects which have very steep hard X-ray spectra. On the other hand, the soft photons which comprise $l_{s}$ may be emitted predominately in the unobservable EUV, and may not be directly related to the $A S C A$ band soft excess. It is interesting to recall that the EUV photons are those that should be responsible for photoionization of the broad-line region clouds, rather than the soft excess photons seen in the $A S C A$ or ROSAT band. If we accept the model proposed by Wandel \& Boller (1998), that the narrowness of the lines is a consequence of a very strong but unobservable EUV emission, the observed correlation between hard X-ray photon index and $\mathrm{H} \beta$ FWHM would support a difference in $l_{s}$ as the origin of the steep hard photon index, rather than in geometry of the Comptonizing electrons.

A yet unresolved point is the fact that soft X-ray selected samples are composed of half NLS1s but the other half are broad-line Seyfert 1 galaxies (Grupe et al. 1999a). The presence of broad-line Seyfert 1 galaxies in soft X-ray selected samples means that they too have strong soft excess components. It is not yet known whether or not these the soft X-ray selected broad-line objects have systematically steeper or flatter hard X-ray spectra than hard X-ray selected Seyfert galaxies; however, there is evidence that at least some of them have very flat hard X-ray spectra (e.g. 1H 0419-577 has a hard X-ray photon index 1.5; Guainazzi et al. 1998b). Again, this may mean that the bulk of the soft photons are emitted in the unobservable EUV and we don't know how strongly peaked the spectrum is in that region. Some information may come from examination of UV line ratios from soft X-ray selected AGNs as they may be sensitive to the shape of the EUV ionizing continuum (Krolik \& Kallman 1988; Zheng, Kriss \& Davidsen 1995; Korista, Ferland, \& Balwin 1997). This work must be done carefully, however, as difference in radial or density distribution of emitting clouds can also modify line ratios.

\subsubsection{Nonthermal Comptonization}

It has recently been discovered that in the soft state, the gamma-ray spectrum of the Galactic black hole candidate Cyg X-1 extends as a power law to at least $1 \mathrm{MeV}$ without a break (Phlips et al. 1996). Such high energy emission is unlikely to be produced in a thermal plasma, it has therefore been asserted that this is evidence for nonthermal Comptonization in the soft state of Cyg X-1 (Gierliński et al. 1998). Following the analogy that NLS1s may be Seyfert galaxies in the soft state implies that nonthermal Comptonization may be occurring in NLS1s.

Gierliński et al. (1998) apply a hybrid thermal/nonthermal model to Cyg X-1 (see also Coppi 1999 for a review of hybrid models). The nonthermal part is comprised of electrons with a steep 
power law distribution, and the optical depth is sufficiently low that the soft photons experience at most 1 scattering. Therefore, the photon index of the radiation will be the same as that of the electrons, and in principle a photon index of 2.4 can be produced easily. The required electron distribution may be natural as it is the same one that cosmic rays have. This model is to be distinguished from the saturated nonthermal Comptonization, which produces photon indices $\leq 2$ (e.g. Swensson 1994). Thermal electrons can be present also, if the time scale for energy loss due to Coulomb interactions is shorter than the Comptonization time scale. They can also Compton-scatter soft photons and produce the usual thermal spectrum.

\subsubsection{Bulk Motion Comptonization}

A final model that has been suggested for NLS1s is that of bulk motion Comptonization (Shrader \& Titarchuk 1998). This model assumes a three part accretion flow consisting of a cold Keplerian optically thick accretion disk and a sub-Keplerian optically thin corona, both of which suffer a shock at 10-30 $R_{S}$. Interior to the shock, the flow is hot and the optical depth is $\sim 1$. The hot plasma interior to the shock will have a high inflow velocity. It will intercept soft photons from the optically thick material exterior to the shock and upscatter them by transferring kinetic energy rather than thermal energy. The predicted hard X-ray spectrum will have a steep slope with $\alpha \sim 1.5$ (Chakrabarti \& Titarchuk 1995; also Ebisawa, Titarchuk \& Chakrabarti 1996). Bulk motion Comptonization is also discussed by Colpi (1988). A diagnostic of this model is that the spectrum should not extend beyond $511 \mathrm{keV}$; thus it cannot be applied to Cyg X-1 in the soft state because the observed spectrum extends to $1 \mathrm{MeV}$.

In this model, it is asserted that the hard X-ray spectrum will be weak. This is at least partially because the intensity of the soft X-ray photons that the infalling electrons see will be decreased by special relativistic effects (e.g. Pietrini \& Krolik 1994). Also, some of the upscattered

photons may possibly be trapped by the flow and not be able to diffuse out (e.g. Colpi 1988). This scenario may not be appropriate for NLS1s; Grupe et al. (1998a) finds that soft X-ray selected AGN, which are comprised of $50 \%$ NLS1s, are stronger in soft X-rays rather than being weaker in hard X-rays. However, there is some evidence that the hard power law is weaker in optically selected samples (Laor et al. 1997a).

\subsection{The Soft Excess}

This paper is the first to systematically study the soft excess in a moderate number of $A S C A$ observations from NLS1s. Evidence for a soft excess was found in 17 of the 19 objects without significant absorption, implying that it is nearly ubiquitous in NLS1 ASCA spectra. The temperature of the black body used to parameterize the soft excess was nearly the same for all objects. However, an important result is that there is a wide range of soft excess strengths. 
At $0.6 \mathrm{keV}$, a factor of 50 is spanned, as measured by the black body parameter (Section 3.2). Note that in principle this result could be the effect of a hard X-ray power law with a range of strengths, or an inverse correlation between strength of the soft excess and weakness of the hard power law. These possibilities cannot be distinguished on the basis of the ASCA data alone. Another important result is that the strength of the soft excess is correlated with measures of X-ray variability: objects with strong soft excesses tend to have higher excess variance and skew parameter than objects with weak soft excesses. There appears to be no correlation between the soft excess strength and optical emission line parameters, suggesting that this behavior is not connected with Boroson \& Green (1992) Eigenvector 1.

\subsubsection{The Soft Excess and the Optical Emission Lines}

A strong soft excess is predicted to affect the thermal balance in the two phase model of the broad-line region, resulting in a lower temperature of the hot intercloud medium (Fabian et al. 1986). They predict that this will result in a very small region of the thermal stability curve being amenable to two phases. While a stationary two-phase medium in pressure balance may not be a completely appropriate description for the broad-line region clouds, this result may be partially responsible for the lower equivalent widths of permitted emission lines (e.g. Goodrich 1989) and the correlation between photon index and equivalent width observed in NLS1s.

The strong soft excess may also affect the location of the broad line region. Wandel \& Boller (1998) note that if the steep soft ROSAT spectra are extrapolated into the unobservable EUV, a stronger ionizing continuum is inferred. If the permitted lines form at a particular ionization parameter, a larger radius for the broad-line clouds would result. The Keplerian velocities at larger radii will be smaller, and therefore the lines will be narrower. Alternatively, the densities in the broad line region clouds could be larger. Such an idea may explain correlation of the density-indicating ratio Si III]/C III] with Fe II/H $\beta$ recently presented by Wills et al. (1999).

\subsubsection{The Soft Excess Temperature}

The soft excess is conventionally thought to be the high energy tail of the accretion disk spectrum. If so, a soft excess at higher temperatures is expected for larger accretion rates and smaller black hole masses (Ross, Fabian \& Mineshige 1992). Drawing upon an analogy with Galactic Black Hole candidates in their high state, and using RE 1034+39 for their example, Pounds, Done \& Osborne (1995) claim that the soft excess could be primary emission from an accretion disk at near Eddington luminosity. They note that the relation of inner disk temperature

and black hole mass for two objects radiating at near the Eddington limit is $T_{1} / T_{2}=\left(M_{2} / M_{1}\right)^{\frac{1}{4}}$, and that the observed temperature ratio is consistent with plausible differences in the black hole mass of RE $1034+39$ and the BHC Cyg X-1. 
As noted above and in Section 3.2, the temperature of the soft excess measured among NLS1 is nearly constant, despite large differences in luminosity. RE 1034+39 and PHL 1092 have, respectively, the lowest and highest luminosities of the six strong soft excess objects in this sample. The difference in luminosity is a factor of 30, suggesting that the black hole mass is a factor of 30 higher in PHL 1092. If so, according to thin disk models, the temperature at the inner edge of the accretion disk should be a factor of 2.3 lower in PHL 1092 compared with RE 1034+39; in fact, it is slightly higher. A soft excess with $k T$ a factor of 2.3 lower would not appear in the $A S C A$ band pass. However, it must be noted that there could be systematic uncertainties with the measurement of the temperature of the soft excess because it is a continuum component appearing at the edge of the $A S C A$ bandpass, and there could be some model dependence. Furthermore, simulations show that there can be a tendency for very weak soft excesses to have a higher measured temperature.

This temperature constraint becomes even stiffer when ROSAT results are considered. In this study, a significant correlation was found between the ROSAT photon index and the soft excess strength, implying that objects with strong soft excesses generally have very steep ROSAT spectra. Grupe (1996) found a significant correlation between the photon index in a soft X-ray selected sample and the redshift. High redshift objects are more luminous in count rate limited samples. All of may imply that there could be objects which are even more luminous than PHL 1092 which have soft excesses observable in the $A S C A$ band; however, spectroscopic observations would be necessary to confirm the temperature.

\subsubsection{Normalization of the Soft Excess}

The soft excesses were modeled using a blackbody. Assuming that the emission originates in an accretion disk, one may ask whether the size of the emission region implied by the strength of the black body is commensurate or at least smaller than the source size inferred using variability arguments.

Using $H=50 \mathrm{~km} / \mathrm{s} / \mathrm{Mpc}$, the area of the emission region was computed from the black body model fits for all of the objects which had detectable soft excess component. The areas ranged from $10^{+20.7} \mathrm{~cm}^{-2}$ for NGC 4051 to $10^{24.8} \mathrm{~cm}^{2}$ for RX J0439-45. The areas were also quite large for PHL 1092, PG 1211+143 and PG 1404+226, which were among the most luminous objects in the sample. Rapid variability was observed from RX J0439-45: a change in flux by a factor of $>2$ was detected in about 2700 seconds. If the emission occurs within $7 R_{S}$, then the upper limit on the black hole mass would be $4 \times 10^{7} M \odot$. However, this exercise implicitly assumes that the emission is not coming from the entire central region but rather a localized emission region, and therefore, the light crossing time of the source may overestimate the size of the emission region. Nevertheless, for illustration, consider the emitting region for the $A S C A$ soft excess to be between 7 and $8 R_{S}$. Then the area of that emitting region for a $4 \times 10^{7} M \odot$ black hole would be $6.8 \times 10^{27} \mathrm{~cm}^{2}$. This means that the time scale of variability for RX J0439-45, combined with its 
soft excess normalization, imply that only $0.1 \%$ of the region need be emitting if the whole thing is optically thick.

However, in the classical the thin disk model, the temperature of the disk at $7 R_{S}$ should be much cooler than the temperatures of the soft excesses observed here. This may be a consequence of the fact that the temperatures in the thin disk model are obtained by averaging in the vertical direction. When realistic radiative transfer is taken into account, it turns out that the underlying disk emission will be Compton upscattered by the hotter upper layers of the disk and the observed emission should be hotter (Czerny \& Elvis 1987; Ross, Fabian \& Mineshige 1992; Shimura \& Takahara 1993). Compton scattering will smear the local disk black body over a larger band pass, and therefore a larger area will be required to emit the observed soft excesses. An estimation of the increased area needed can be obtained from Figure 6 in Ross, Fabian \& Mineshige (1992), which shows the fraction of total disk emission in soft X-rays above $150 \mathrm{eV}$ as a function of black hole mass and accretion rate. For a $4 \times 10^{7} M_{\odot}$ black hole, the fraction of flux in soft X-rays is order $10 \%$ if the Eddington fraction is as high as $1 / 3$, implying that an area at least ten times larger than the previous estimate is required; this would still be only $1 \%$ of the $7-8 R_{S}$ annulus. For larger black hole masses and smaller Eddington fractions, the fraction emitted in soft X-rays is smaller.

\subsubsection{Models of the Soft Excess}

Accretion disk models predict more intense X-ray emission as well as higher inner edge temperature when the accretion rate is high (e.g. Shakura \& Sunyaev 1973). However, while standard models of accretion disk emission find a good fit to the optical-UV continuum, they predict far too cool temperatures at the inner edge to explain the soft X-ray emission. Many of these models ignore the vertical structure in the accretion disk and the radiation transfer. More sophisticated treatments of these corrections indeed do find that the accretion disks can emit strongly in soft X-rays, especially when the accretion rate is high and/or the black hole mass is low (e.g. Czerny \& Elvis 1987; Ross, Fabian \& Mineshige 1992; Shimura \& Takahara 1993). A shifted and strengthened accretion disk spectrum can explain the steeper ROSAT spectra observed in NLS1s, and therefore this was interpreted as evidence for a high accretion rate (e.g. Boller, Brandt \& Fink 1996; Laor et al. 1997a; Grupe et al. 1999a). This can also generally explain the high temperatures and higher frequency of X-ray soft excesses observed in the $A S C A$ spectra from NLS1s (e.g. Pounds, Done \& Osborne 1996).

More realistic accretion disk models can also potentially explain the fact that the temperature of the X-ray soft excess remains constant over a large range of source luminosities and inferred black hole masses (Section 4.2.2). Examining a wide range of parameters, Shimura \& Takahara (1993) solve for hydrodynamic equilibrium and radiative transfer self-consistently . They find that the emission spectrum gradually shifts toward the soft X-rays as the accretion rate increases, confirming the results of Ross, Fabian \& Mineshige (1992). However, when the accretion rate 
is very large, nearly the Eddington rate, the derived temperature, near 100-200 eV, has only a weak dependence on the black hole mass. This constancy in spectral shape occurs because at such high accretion rates, the scattering optical depth becomes independent of black hole mass, Comptonization is the dominant process at the inner edge of the disk, and the Compton $y$ parameter in the upper layers of the disk becomes larger than 1 and nearly independent of black hole mass. This behavior is not expressed for lower accretion rates, where the soft X-ray cutoff is a stronger function of temperature.

The large range in soft excess strengths may suggest that the soft excess does not have a uniform origin in NLS1s. Another possible origin besides primary disk emission is suggested by the discovery of soft X-ray line emission near $1 \mathrm{keV}$ in two of the weak soft excess objects, PG 1244+026 (Fiore et al. 1998) and Ark 564, as well as the ionized Fe K $\alpha$ line in Ton S180. The soft excess in these objects could be due to reflection from an ionized accretion disk. When the surface of the accretion disk becomes ionized, the reflectivity to soft X-rays is increased as the light elements are ionized and the absorption in the upper layers is decreased. This situation has been explicitly modeled by Matt, Fabian \& Ross (1993a) and Życki et al. (1994) and discussed by Czerny \& Życki (1994) and Fiore, Matt \& Nicastro (1997). Fiore, Matt \& Nicastro (1997) mention that the effect of the reflection by ionized material would be an increase in the steepness of the $0.2-2 \mathrm{keV}$ spectrum by about $\Delta \Gamma \approx 0.3$.

Another possibility is that the weak soft excesses seen in some NLS1s is the signature of Compton scattering of blackbody emission (e.g. Nishimura, Mitsuda \& Itoh 1986). Evidence for this feature was found in the high state spectrum from Cyg X-1 (Cui et al. 1998; Gierliński et al. 1999). The spectrum was not adequately modeled with a black body plus power law; an additional soft excess, a signature of the fact that a black body is the source of photons, was required for intermediate energies. If the weak soft excess observed in some objects is in fact Comptonized blackbody emission, then the primary black body must not be very far below the $A S C A$ bandpass.

\subsection{The Warm Absorber}

This paper presents the first systematic survey of the warm absorber properties of NLS1s. The first result is that there is evidence that the incidence of warm absorbers, as indicated by the presence of O VII and O VIII edges, is lower in NLS1s. The second result is that when warm absorbers are detected, they appear to have a lower ionization state than those in Seyfert 1 galaxies with broad optical lines. Both of these conclusions, however, are subject to restrictions based on the methods used to search for the warm absorber. 


\subsubsection{Biases}

Neither the narrow-line Seyfert 1 sample considered here, nor the broad-line Seyfert 1 comparison sample presented in Reynolds 1997, can be considered complete in any way. Therefore, it is important to determine whether biases in the sample selection could influence the result.

The NLS1s discussed here are selected in several ways, all of which may be biased against discovering highly absorbed objects. Soft X-ray selected objects present the strongest bias. They are drawn from Grupe's ROSAT soft X-ray selected AGN (e.g. Ton S180, RX J0439-45, RE 1034+39, IRAS 13349+2438); the ROSAT spectra from these objects indicates little evidence for heavy absorption (Grupe et al. 1998b). They are also drawn from the Moran, Halpern \& Helfand (1996) IRAS/ROSAT cross correlation sample (e.g. IRAS 13224-3809, IRAS 17020+4544, IRAS 20181-2244). Two objects were selected by the HEAO A-1 experiment (PKS 0558-504 and $1 \mathrm{H}$ 0707-495). Other objects are drawn from PG quasars and Markarian galaxies, including Mrk 335, I Zw 1, Mrk 142, PG 1211+143, Mrk 766, PG 1404+226 and Mrk 478. These objects are chosen by their blue optical spectra, which means that objects with moderate reddening may be present, but objects with very heavy absorption should not be.

In contrast, many of the broad-line Seyfert 1 galaxies considered by Reynolds 1997 are members of the HEAO-A2 Piccinotti sample (Piccinotti et al. 1982). HEAO-A2 was sensitive between 2 and $10 \mathrm{keV}$, so many more absorbed sources are present in the Piccinotti sample. Thus the difference in selection could lead to a bias in the warm absorber result. On the other hand, no NLS1s are present in the Piccinotti sample, suggesting that hard X-ray selection is biased against these objects, whether or not they are absorbed. That may be because the steeper hard X-ray spectrum of NLS1s make them somewhat weaker in the 2-10 keV band compared with broad-line Seyfert galaxies.

There is also a possibility of a spectroscopic bias. It is possible, although it has not yet been demonstrated, that warm absorbers and associated polarization is found more often among Seyfert 1.5 galaxies compared with Seyfert 1s. Intermediate-type narrow-line Seyfert 1 galaxies might be difficult to identify without good signal-to-noise spectra. A case in point is IRAS 20181-2244; it was thought to be a Seyfert 2 galaxy until good signal to noise spectroscopy allowed clear identification of the Fe II emission (Halpern \& Moran 1998).

It is difficult to determine what kind of sample would be the best one in which to study the absorption properties in NLS1s and broad-line objects in an unbiased way. Hard X-ray selected samples should be ideal, since they choose objects whether or not they are absorbed. However, the lack of NLS1 in the Piccinotti sample make this impossible. Spectroscopically identified Seyfert galaxies may present the best sample, since both absorbed and unabsorbed Seyfert 1s and NLS1s are present. 


\subsubsection{The Frequency of the Warm Absorber in NLS1s}

Despite the potential selection bias, there are also some physical reasons to explain why a warm absorber may be less frequent in NLS1s. The simplest explanation is that it is a consequence of the steep X-ray spectrum. There will be fewer photons above the threshold energy for oxygen ionization if the spectrum is steep, and therefore, fewer appropriate ions may be created. Note that this may not be the whole story; if the warm absorber occurs in equilibrium gas, then the thermal and ionization balance would be more important for determining the properties of the warm absorber.

\subsubsection{The Ionization State of Warm Absorbers in NLS1s}

The potential biases explored above should not affect the second result of this study, that the ionization appears to be lower in warm absorbers in NLS1s compared with Seyfert 1 galaxies with broader optical lines. Recall that this inference is based on the comparison of distributions of O VII and O VIII edges between the NLS1s presented here and a sample of broad-line objects from Reynolds (1997): while the distributions of O VII edge depths were indistinguishable, detection of significant optical depth in the O VIII edge was less frequent in the NLS1s. This can be inferred to be evidence that the ionization parameter is lower in NLS1s. However, there are several weaknesses in this argument. In a single-zone model, the oxygen edges are not independent. Therefore, it is conceivable that a detailed study of the fractional ionization of the gas as a function of ionization parameter will reveal that for NLS1s the $\mathrm{O}^{+7}$ inhabits only a very narrow range of ionization parameter, and the gas is more typically characterized by $\mathrm{O}^{+6}$ and fully ionized oxygen. Furthermore, assuming that the gas is in multiphase equilibrium, it may happen that $\mathrm{O}^{+7}$ is the dominate ionization state under conditions when the gas is unstable. Both of these questions require computations beyond the scope of this paper to address. Finally, the two-edge model will fail to detect very high ionization warm absorbers which may be dominated by Fe L resonance absorption and have been postulated to be present in several strong soft excess NLS1s by Nicastro, Fiore \& Matt 1999.

Among Seyfert 1 galaxies with broad optical lines, there is some evidence that the ionization of the warm absorber is about the same for all objects. George et al. 1998 found, in their study of a sample of $23 A S C A$ observations of 18 objects dominated by broad-line Seyfert 1 galaxies, that the ionization parameter appears to be strongly peaked around one value, although a maximum likelihood analysis indicates a significant dispersion. This result may be due either to a sample selection effect or a physical selection effect. Possible reasons why it may be a selection effect were argued by George et al. They postulate that if the absorbing gas with lower ionization parameter happens to always be coincident with dust, the dust may block the view to the broad line region clouds causing the objects with low ionization parameters to be classified as Seyfert 2s which would have been excluded from the George et al. sample. The results presented here suggest that 
Seyfert 1 galaxies with less ionized warm absorbers exist.

The typically steeper X-ray spectrum may affect the conditions of the warm absorber in NLS1s. Reynolds \& Fabian (1995) consider models in which the warm absorber is in thermal equilibrium with the broad-line region clouds. They show that the presence of a soft excess or a steeper X-ray spectrum appears to modify the thermal stability curve such that the warm absorber is stable over a broader range of parameters. Everything else being equal, this would predict that warm absorbers should be more common in NLS1s. At a given temperature, the values of $\xi / T$ are generally larger for steeper spectra and stronger soft excesses (see Reynolds \& Fabian 1995), suggesting that the ionization should be larger in NLS1s rather than smaller. This is in contrast to what is inferred from the oxygen edge measurements but may be consistent with the presence of a very highly ionized warm absorber.

A similar result is presented by Shields, Ferland \& Peterson (1995). They investigated the contribution of optically-thin broad-line region gas in photoionization but not thermal equilibrium in Seyfert 1 galaxies, and find that optically thin gas may contribute substantially to the high ionization line emission in AGN. The predicted opacity and some ionization states of this gas is also appropriate to produce the warm absorber phenomenon. Shields et al. find that when there are more soft X-rays relative to UV, the optically thin material is in general more highly ionized and will more likely appear as a warm absorber. Again, this predicts a more prevalent warm absorber in NLS1s, in contrast to what is inferred.

These models assume that the warm absorber gas is in ionization and sometimes also thermal equilibrium. That may not be the case. Krolik \& Kriss (1995) and Nicastro et al. (1999) consider in some detail the situation in which the warm absorber gas is subject to a time variable photoionizing source. Since each ion has a different recombination rate, the details can be complicated. However, when the time scale of variability is shorter than the recombination time scale, the warm absorber will be overionized with respect to ionization equilibrium, generally speaking. This would predict an even higher ionization state than predicted in equilibrium models.

This discussion shows that many simple one-zone models cannot easily explain the lower ionization inferred from the oxygen edge measurements in the sample of NLS1s. However, two-zone models may be able to explain this result naturally. Evidence for more than one zone of ionized material has been found in a few well-studied broad-line Seyfert galaxies. Difference in variability behavior of the O VII and O VIII edges in response to flux changes in MCG-6-30-15 provides one example (Otani et al. 1996), and discrepancies between absorption profiles in high optical depth warm absorbers and detailed single zone models was also interpreted as evidence for two absorbers (George et al. 1998). Evidence of an association of the outer warm absorber with dust via reddening (Reynolds 1997; Reynolds et al. 1997) and polarization (Leighly et al. 1997b) placed the outer warm absorber coincident with or exterior to the broad line region. Therefore, one possibility is that the inner warm absorber in NLS1s may be too highly ionized to be observed. Alternatively, it may not exist. For example, it may have been blown from the nucleus by 
radiation pressure. If only the outer warm absorber is detected, the cumulative ionization state will appear to be low. This somewhat fits our expectations because a fairly good correlation between polarization and presence of a warm absorber is observed in this sample; the dusty warm absorber should be the outer one, as dust is not expected to survive the intense heating interior to the broad-line region.

We may be able to test whether or not there is warm absorber gas present in NLS1s, and in similar quantities as in broad-line Seyfert 1 galaxies, by measuring emission lines, such as coronal lines, that are thought to associated with warm absorber gas (e.g. Porquet et al. 1998; Erkens, Appenzeller \& Wagner 1997). A complicating factor may be that the ionization potentials for the common coronal line ions are quite high, around $0.2 \mathrm{keV}$. NLS1s generally have steeper soft X-ray spectra than Seyfert 1s with broader optical lines, and there is evidence that NLS1s are stronger on average at $0.2 \mathrm{keV}$ than broad-line Seyfert 1s compared with the rest of the spectrum (Grupe et al. 1998a). Therefore, stronger coronal line emission might be expected from NLS1s, independent of whether warm absorber gas is present. This expectation appears to be roughly borne out in simulations by Porquet et al. Of the two continuum models used, the one that is stronger in soft $\mathrm{X}$-rays requires a lower column density to produce the observed coronal line equivalent widths, at least for the lower densities. Interestingly, little dependence of the oxygen edges on the continuum shape is found.

\subsection{Models for NLS1s}

\subsubsection{Models Based on Orientation}

NLS1s have narrower optical emission lines than Seyfert 1 galaxies with broad lines. It has been suggested that the origin of this behavior is that NLS1s are viewed with a face-on (pole-on) orientation. Narrower emission lines are seen because the optical line emitting clouds are confined to a plane and therefore a smaller degree of Doppler broadening is seen (e.g. Osterbrock \& Pogge 1985; Puchnarewicz et al. 1992; Boller, Brandt \& Fink 1996). Stronger soft X-ray emission is expected for face-on orientations from some geometrically thick accretion disk models (Madau 1988). Models in which observed line width is based on orientation have recently been supported by evidence for a correlation between radio power and the monochromatic optical luminosity (an indication of the orientation angle) and the width of the $\mathrm{H} \beta$ lines (Wills \& Brotherton 1995). It has been postulated that the Fe II emission may arise in the accretion disk (Collin-Souffrin, Hameury \& Joly 1988; Kwan et al. 1995), so a face-on viewing angle would enhance the amount of Fe II observed.

To be consistent with the $A S C A$ results, the observed hard X-ray photon indices should be orientation dependent. The slab thermal Comptonization model as implemented by Haardt \& Maraschi (1993) predicts softer spectra as the inclination angle increases. This effect originates in the anisotropy of the first order scattering, resulting in more photons directed downward toward 
the slab. A similar result was obtained by Dove, Wilms \& Begelman (1997) using a similar but improved slab model. This trend is opposite of what the observations require. When this model is generalized, and the hot phase is assumed to be in localized regions on the disk, the dependence on inclination decreases, and the index depends rather on the height of the hot phase blobs above the disk.

A face-on geometry might explain the low frequency of warm absorbers in NLS1s inferred from this sample. It has been suggested that intermediate Seyfert galaxy spectra are produced when the line of sight to the broad line region is partially blocked by absorbing material (e.g. Lawrence \& Elvis 1982). The absorbing material may be the molecular torus thought to lie at a distance intermediate to the broad and narrow-line regions; the warm absorbing material and associated dust may be ablated from the molecular torus. Thus, warm absorbers may be more likely to be observed when the inclination is moderate rather than face-on.

The enhanced variability observe in NLS1s should also be orientation dependent. These aspects are discussed in Part 1, and both face-on and edge-on scenarios may be able to explain the enhanced variability. The edge-on model may naturally explain the fact that the variability parameters are correlated with the strength of the soft excess, since in some disk models, enhanced soft X-ray emission is expected when the viewing angle is edge-on (Laor \& Netzer 1989).

\subsubsection{Models Based on Accretion Rate and Black Hole Mass}

It has been suggested that a higher accretion rate relative to Eddington in NLS1s can explain many of their characteristic properties. If so, assuming that the efficiency of conversion of accretion energy to radiation is the same in both types of objects, the black hole mass should be smaller in NLS1s. The optical lines then will be narrower, assuming that the broad-line region emission is the same and that the motions of the broad line emitting clouds are dominated by Keplerian velocities. However, it may be more important that a high accretion rate implies an enhanced photoionizing continuum (Wandel \& Boller 1998).

Several models of enhanced accretion predict a strong and hot soft excess, as discussed in Section 4.2.3. That an enhanced accretion rate should be associated with a steeper hard X-ray spectral index was first introduced first by Pounds, Done \& Osborne 1995. If the geometry and high energy cutoff are assumed to be the same in all Seyfert 1 galaxies, then we can determine how much larger intrinsic luminosity of the soft component would have to be. It was shown in Section 4.1.1 that $l_{s} / l_{h}$ should be a factor of 3.8 larger in NLS1s to explain the difference in slope. Assuming a covering fraction of 0.5 and using Eq. 29 from Pietrini \& Krolik (1995), the intrinsic soft X-ray luminosity would be a factor of 5.5 larger in NLS1s. However, the steeper hard X-ray spectrum may also mean that the hot phase luminosity is smaller in NLS1s. Assuming that the

normalization at $1 \mathrm{keV}$ is the same in both types of objects (Grupe et al. 1998a), and the spectra show an exponential cutoff at $100 \mathrm{keV}$, the hot phase luminosity in NLS1s may be $70 \%$ that of 
broad-line objects. Therefore, the intrinsic luminosity in the soft component would have to be about 7.5 times greater in NLS1s, assuming that everything else remains the same. If the efficiency of conversion of gravitational potential energy to radiation is the same in both types of objects, this would mean that the accretion rate should be about 7.5 times greater in NLS1s compared with broad-line objects.

As discussed in Part 1, the excess variance versus luminosity plot can also be explained by a higher accretion rate and correspondingly smaller black hole mass, assuming that the variability has the same structure in NLS1s as in Seyfert galaxies with broad optical lines. The inferred difference is a factor of 10, interestingly near the enhancement required to produce the steep hard X-ray spectrum discussed above.

The high accretion rate may also affect the properties of the warm absorber. Reynolds \& Fabian 1995 hypothesize a dynamical model for the warm absorber in which neutral gas is accelerated by radiation pressure on the resonance lines. In the process, the gas is partially ionized. When the gas becomes ionized, the acceleration ceases, the inward gravitational acceleration takes over until it becomes compressed enough to recombine. The critical luminosity required to balance these two effects is estimated by Reynolds \& Fabian (1995) to be about $0.05 L_{E d d}$. They speculate that if the luminosity exceeds this critical luminosity, an outflow of highly ionized material may be formed, and this may explain the lower frequency of warm absorbers in quasars, as they are thought to be radiating at a higher Eddington fraction than Seyfert galaxies.

\subsubsection{Other Comments on Physical Models}

The two phase model has been a very successful scenario to describe the behavior of broad-line Seyfert galaxies. In this model, most of the accretion energy, rather than being released in the disk, is dissipated instead in the corona above the disk (Haardt \& Maraschi 1991, 1993; Stern et al. 1995; Svensson \& Zdziarski 1994). An important feature of this model is that if a sufficient amount of energy is released in the corona, the thin disk solution can exist close to the black hole (Svensson \& Zdziarski 1994); otherwise, a transition to another type of accretion is expected.

This model assumes that the source of power for the disk emission is reprocessing of the coronal emission by the optically thick material. However, in NLS1s, the soft excess is sufficiently strong and the hard X-ray photon index is sufficiently steep that the soft excess cannot be powered by reprocessing (e.g. Pounds, Done \& Osborne 1996). Although it has been shown that a thin disk with intrinsic emission can exist close to the black hole in Galactic black hole candidates (Gierliński et al. 1999), because of the dependence of the stability criterion on black hole mass as well as accretion rate, there can be no stable AGN thin disk solution in this situation. This fact marks a limitation of the BHC/AGN analogy as an explanation for NLS1 behavior. However, it may alleviate one of the more uncomfortable problems with this analogy. As has been widely demonstrated, NLS1s are more variable than broad-line Seyfert galaxies; in contrast, BHC in the 
soft state are less variable than in the hard state.

What kind of accretion flow might be expected at small radii when the radiation pressure becomes too large for the thin disk solution? One possibility that has been proposed for hard state Galactic black hole candidates and broad-line radio galaxies is that the inner regions form a hot, optically thin, geometrically thick torus, either the two-temperature torus (SLE; e.g. Shapiro, Lightman \& Eardley 1976) or an advection dominated flow (ADAF; for a review, Narayan, Mahadevan \& Quataert 1999). These flows are typified by rather hard X-ray power-law emission and no soft excess; therefore they are likely not to be applicable to NLS1s.

It was originally thought that when the accretion rate is high, there would be a radiation pressure dominated disk (e.g. Shakura \& Sunyaev 1973). However, this solution is shown to be unstable if the viscosity is proportional to the radiation pressure, although it may be stable if the viscosity is proportional to the gas pressure only (Lightman \& Eardley 1974). At higher accretion rates lies the slim disk solution, in which part of the energy is advected into the black hole (e.g. Abramowicz et al. 1988). In NLS1s, it is possible that the accretion rate is in the radiation dominated region and it instead develops an unsteady flow. Such a scenario has recently been proposed for the superluminal black hole binary GRS $1915+105$ by Belloni et al. 1997. This object may undergo unsteady accretion in a similar way as dwarf novae, except the Lightman-Eardley instability (which operates between the thin disk and slim disk solutions, with the unstable radiation pressure dominated disk in between) operates rather than the thermal-viscous instability. The time scales for the limit cycle are much longer for AGN (on the order of years); however, on shorter time scales, during the accreting portion of the cycle, the accretion happens unsteadily, and rapid variability is observed as heating fronts move through the disk at the sound speed, and material is removed on the infall time scale (Belloni et al. 1997).

Such a scenario may be attractive for NLS1s. Slim disks can produce soft X-rays (Szuszkiewicz, Malkan \& Abramowicz 1996). The sound crossing speed and infall time scales in the inner region may be appropriate for the rapid variability observed in NLS1s. The heating waves could conceivably be coherent, and therefore produce large amplitude flares. The longer time scale variability due to the limit cycle behavior may provide a way to explain the very large amplitude variability observed from some NLS1s (IC 3599: Grupe et al. 1995a; WPVS 007: Grupe et al. 1995b; RX J0134-42: Grupe et al. 1999b).

The correlation between the excess variance and strength of the soft excess might be explained as a consequence of a variable transition radius $r_{t}$ from the usual AGN geometry, that is, a thin disk plus corona from magnetic flares existing at $r>r_{t}$, to the proposed highly variable unsteady inner disk at $r<r_{t}$. The location of this transition radius may be a function of specific accretion rate. When $r_{t}$ is small, only a relatively small region is characterized by the highly variable unstable disk, while there is a relatively large region containing the usual thermal corona present in broad-line objects. Therefore, the soft excess is moderately strong, and the hard power law is relatively strong, and, while the excess variance is higher than that of broad-line Seyfert 1 
galaxies, the variability is not detectably non-Gaussian because the coherent variability from the inner region is diluted by stochastic variability from the flaring outer region. When $r_{t}$ is large, the highly variable unstable inner disk dominates the central parts of the AGN, leading to a very strong soft excess. The hard tail is weaker, because less of the accretion energy goes into the usual thermal corona. Because the variability is dominated by the very variable inner region, the excess variance is again large, but since the coherent variability is minimally diluted, non-Gaussianity is detected. Note that because the region sizes change approximately in concordance with the emission from each region, the compactnesses may stay approximately the same.

\section{Summary and Future Observations}

I present a comprehensive and uniform analysis of $25 A S C A$ observations from 23 Narrow-line Seyfert 1 galaxies. The results of spectral analysis and correlations are reported here; the results of the time series analysis are reported in Part 1. The primary results of this paper are the following:

- A maximum likelihood analysis confirms that the hard X-ray photon index is significantly steeper at $>90 \%$ confidence in this sample of NLS1s than in a random sample of Seyfert galaxies with broad optical lines. As previously noted, this can be explained by two-phase thermal Comptonization models if the soft photon input is greater in NLS1s than in broad-line objects. This view may be supported by the observed correlation between the photon index and the $\mathrm{H} \beta$ FWHM among the objects in this sample. Alternatively, the steep photon indices may originate in a single scattering from a power law distribution of electrons.

- Soft excess emission was detected in 17 of the 19 objects which had no significant absorption. While the luminosities span two orders of magnitude, the temperatures are roughly consistent, a result that contradicts predictions of the simplest thin disk model. The soft excess strength above the power law spans a large range (a factor of 50), and the strength of the soft excess was found to be correlated with the variability parameters as well as the ROSAT photon index. Possible origins for the soft excess include primary emission from an accretion disk characterized by a high accretion rate, reprocessing in an ionized disk, and the signature of Comptonization of black body emission.

- Evidence was found that the warm absorber is less likely to be observed in NLS1s and when present has typically lower ionization than in broad-line Seyfert 1 galaxies. However, this result was obtained using a two-edge parameterization which would not necessarily be sensitive to warm absorbers with very high ionization. This result may imply that the inner warm absorber is either absent or too highly ionized to be detected in these objects, but the outer warm absorber, typified by a lower ionization parameter, remains.

- The iron line equivalent width average and distribution was found to be the same in the sample of NLS1s as in a random sample of Seyfert 1 galaxies with broad optical lines, a 
result which may imply that the amount of reprocessing is the same in both types of objects. However, this result is qualified by the generally poorer statistics at the iron line in the NLS1 sample, and the fact that emission from ionized iron, which may have either enhanced or reduced fluorescence yield, was found in a few objects.

Models based on orientation and high accretion rate were examined in the light of these results. A model based a face-on orientation possibly explains the narrow emission lines, stronger soft excess and less frequent warm absorbers, but cannot explain the steep power law, the variability results or the low ionization of the warm absorber. A model based on an edge on viewing angle may be able to explain the variability results, the steep soft excess and perhaps also the steep hard X-ray spectrum but requires NLS1 to consistently be inclined to their host galaxies more than Seyfert 1 galaxies with broad optical lines, and the dominant motion of broad line region clouds would be required to be along the symmetry axis.

A model based on a higher accretion rate relative to Eddington generally fairs the best. It can explain the steep soft excess due to a shift of the accretion disk spectrum to high energies; this also explains the narrow optical emission lines through stronger ionization and also the steep hard X-ray photon index through stronger Comptonization cooling. The rapid variability is naturally explained by the requirement of a smaller mass black hole. The nearly constant soft excess temperature is predicted at high accretion rates in some accretion disk models. The less frequent warm absorber may be explained if it is too highly ionized to be seen or has been blown out of the system.

The ASCA observations and results presented here lead to further questions which may be answerable in the future.

- Observations at higher energies are necessary to make significant progress on understanding the origin of the steep hard X-ray spectrum in NLS1s. Observations up to $20 \mathrm{keV}$ will allow us to search for the Compton reflection component and obtain information on the amount of reprocessing. Observations to higher energies will allow us to determine whether the hard $\mathrm{X}$-ray emission process is thermal or nonthermal. In addition, studies of the photon index spectral variability may yield some constraints on these processes.

- The nature of the soft excess in the $A S C A$ band remains a mystery. Observations with a broader band pass down to $0.1 \mathrm{keV}$ and better statistics would allow us to better constrain the shape. Observations with better energy resolution may reveal line emission characteristic of reprocessing in a ionized disk. Observations of variations in the shape of this component as a function of time also may be valuable.

- Better statistics in the iron line region are needed to determine the importance of reprocessing and also to constrain the inclination and properties of the reprocessing material including ionization and distance from the black hole. 
- Observations with good statistics and energy resolution will allow us to determine the properties of the warm absorber in NLS1s with less ambiguity.

KML gratefully thanks all those people who built and operate $A S C A$. This project could have never attained this form without the help and support of Jules Halpern. Many thanks go to Dirk Grupe for many various kinds of help and advice. Useful discussions with Karl Forster, Julian Krolik, Herman Marshall, Tahir Yaqoob and Andrzej Zdziarski are acknowledged. The following are thanked for a critical reading of a draft: Joachim Siebert, Jules Halpern \& Tahir Yaqoob. This research has made use of the NASA/IPAC extragalactic database (NED) which is operated by the Jet Propulsion Laboratory, Caltech, under contract with the National Aeronautics and Space Administration. This research has made use of data obtained through the High Energy Astrophysics Science Archive Research Center Online Service, provided by the NASA/Goddard Space Flight Center. KML gratefully acknowledges support through NAG5-3307 and NAG5-7261 $(A S C A)$ and NAG5-7971 (LTSA) .

\section{A. Appendix - Notes on Individual Objects}

\section{A.0.4. Ton S180:}

GIS3 5.5-7.5 keV was excluded from spectral fitting.

Turner, George \& Nandra (1998) report evidence for soft X-ray line emission in this $A S C A$ observation of Ton S180. The fact that Turner et al. model the spectra from both CCD detectors and also do not model the soft excess component leads to some doubt about this result. As discussed in Section 2.1, data taken after the beginning of 1996 tended to show large discrepancies between the SIS0 and SIS1 detectors. The Ton S180 observation was made in June 1996 and displays one of the worst examples of this discrepancy found in this sample of data. The SIS1 residuals fall markedly below the SIS0 residuals for energies less than $0.8 \mathrm{keV}$ (Leighly 1998). Thus, spectral complexity appears to be present in the SIS1 detector but not in SIS0. Spectral fitting confirms this conjecture; a soft X-ray line appears significant in the SIS1 spectrum, but there is no evidence for it in SIS0. When a soft X-ray line is added to the best fit model presented here, the improvement in $\chi^{2}$ was 6.2 for 2 d.o.f. and the equivalent width is $7.4 \mathrm{eV}$; therefore this feature is not significant.

\section{A.0.5. PKS 0558-504:}

GIS3 5.0-7.0 keV was excluded from spectral fitting. 


\section{A.0.6. Mrk 766:}

The spectral classification of Mrk 766 has been debated. Osterbrock \& Pogge (1985) classify this object as a Seyfert 1.5; later, however, Goodrich (1989) includes it in his NLS1 spectropolarimetry sample. Goodrich (1989) published a spectrum near H $\beta$ from this object. The typical Lorentzian NLS1 line profile can clearly be seen, and measured FWHM from the plot is $1700 \mathrm{~km} / \mathrm{s}$. I speculate that the Seyfert 1.5 classification arose from a failure of a single Gaussian fit to the Lorentzian profile; this is a common situation in NLS1 optical spectra NLS1s (e.g. Goncalves, Veron \& Veron-Cetty 1998). Furthermore, the H $\beta$ appears weak compared with [O III] in Goodrich 1989; however, this could be a consequence of a Balmer decrement arising from a dusty warm absorber responsible for the ionized absorption in the X-ray band (e.g. Leighly et al. 1997b) and the red optical spectrum (Molendi \& Maccacaro 1994) and not intrinsic to the emitted spectrum.

\section{A.0.7. IRAS $13349+2438:$}

The FWHM H $\beta$ of IRAS $13349+2438$ is $2200 \mathrm{~km} \mathrm{~s}^{-1}$ and therefore it is not an NLS1, technically speaking. However, as noted by Brandt, Mathur \& Elvis 1997, the steep spectrum and variability make it more similar to NLS1s than to Seyfert 1s or quasars with broad optical lines. Therefore it is also included in this sample.

\section{A.0.8. $P G 1244+226$ :}

The soft X-ray line feature reported here is qualitatively the same as that reported by Fiore et al. 1998; however, a smaller equivalent width is reported here (35 compared with $64 \mathrm{eV}$ ). The origin of this difference is that here the spectra over the full $A S C A$ band pass is modeled and a soft excess component is included whereas in Fiore et al., only $0.4-4.0 \mathrm{keV}$ region is modeled and the continuum consists only of a power law. The soft excess component models part of the positive residuals resulting in smaller equivalent width.

The feature in the PG $1244+226$ spectrum was alternatively modeled as an edge at $1.17 \mathrm{keV}$ by Fiore et al. 1998. I found that an edge at $1.17 \mathrm{keV}$ also modeled the spectrum adequately ( $\chi^{2}=487 / 474$ d.o.f.). The unresolved line model yielded a $\chi^{2}$ of 489 for the same number of degrees of freedom, indicating a difference in $\chi^{2}$ of 2 . The likelihood ratio then is just 2.71 and therefore the difference is not significant.

\section{A.0.9. IRAS 17020+4544:}

GIS3 6.0-8.0 keV was excluded from spectral fitting. 


\section{A.0.10. Kaz 163:}

The ratio of data to model indicates that there may be a weak soft excess in this object. However, addition of this component leads to only a small reduction in $\chi^{2}\left(\Delta \chi^{2}=8.7\right)$ and therefore it is required with less than $99 \%$ confidence.

\section{A.0.11. IRAS 20181-2244:}

Marginal evidence for a warm absorber was found during this analysis which was not reported by Halpern \& Moran 1998.

\section{A.0.12. Ark 564:}

Analysis of this object was complicated by the fact that the observation was made partly with a level discriminator and partly without, and the level discriminator was set at different values during the time that it was on. This required different response matrices for the different level discriminator states. Spectra were accumulated excluding the data with the high level discriminator. The spectra were fit simultaneously with spectra from a coordinate $R X T E$ observation. The details will be reported in Leighly et al. in prep. 


\section{REFERENCES}

Abramowicz, M. A., Czerny, B., Lasota, J. P., \& Szuszkiewicz, E., 1988, ApJ, 332, 646

Belloni, T., Méndez, M., King, A. R., van der Klis, M., \& van Paradijis, J., 1997, ApJL, 145

Bergeron, J., \& Knuth, D., 1980, A\&A, 85, 11

Bergeron, J., \& Knuth, D., 1984, MNRAS, 207, 263

Berriman, G., Schmidt, G. D., West, S. C. \& Stockman, H. S. 1990, ApJS, 74, 869

Boller, Th., Bertoldi, F., Dennefeld, M., \& Voges, W. 1998, A\&AS, 129, 87

Boller, Th., Brandt, W. N., \& Fink, H. 1996, A\&A, 305, 53

Boller, Th., Meurs, E. J. A., Brinkmann, W., Fink, H., Zimmermann, U., \& Adorf, H.-M. 1992, A\&A, 261, 57

Boroson, T. A. \& Green, R. F. 1992, ApJS, 80, 109

Brandt, W. N., Fabian, A. C., \& Pounds, K. A., 1996, MNRAS, 278, 326

Brandt, W. N., Mathur, S., \& Elvis, M., 1997, MNRAS, 285, 25p

Brandt, W. N., Mathur, S., Reynolds, C. S., \& Elvis, M. 1997, MNRAS, 292, 407

Brinkmann, W., Kawai, N., Ogasaka, Y., \& Siebert, J., 1996, A\&A, 316, 9

Chakrabarti, S., \& Titarchuk, L. G. 1995, ApJ, 455, 623

Cohen, R. D. 1983, ApJ, 273, 489

Collin-Souffrin, S., Hameury, J.-M., \& Joly, M., 1988, A\&A, 205, 19

Colpi, M. 1988, ApJ, 326, 223

Comastri, A., et al. 1998, A\&A, 333, 31

Comastri, A., Molendi, S., \& Ulrich, M. H., 1997, "X-ray Imaging and Spectroscopy of Cosmic Hot Plasmas", ed. F. Makino \& K. Mitsuda (Tokyo: University Academy Press), 279

Coppi, P. S., 1999, in proc. "High Energy Processes in Accreting Black Holes", eds. J. Poutanen \& R. Svensson, ASP Conf. Series, Vol. 161, p. 375

Corbin, M. R., 1997, ApJS, 113, 245

Cui, W., Ebisawa, K., Dotani, T., \& Kubota, A., 1998, ApJL, 493, 75

Czerny, B., \& Elvis, M., 1987, ApJ, 321, 305

Czerny, B., \& Życki, P. T., 1994, ApJL, 431, 5

Dickey, J. M., \& Lockman, F. J., 1990, Ann. Rev. Astron. \& Astrophys. 28, 215

Dotani T. 1998, ASCA Calibration Report, http://www.astro.isas.ac.jp/ dotani/rdd.htm

Dove, J. B., Wilms, J., \& Begelman, M. C., 1997, ApJ, 487, 747

Ebisawa, K., Titarchuk, L., \& Chakrabarti, S. K., 1996, PASJ, 48, 59 
Elvis, M., Lockman, F. J., \& Wilkes, B. J., 1989, AJ, 97, 777

Erkens, U., Appenzeller, I., \& Wagner, S., 1997, A\&A, 323, 707

Fabian, A. C., Guilbert, P. W., Arnaud, K. A., Shafer, R. A., Tennant, A. F., \& Ward, M. J., 1986, MNRAS, 218, 457

Fabian, A. C., Rees, M. J., Stella, L., \& White, N. E., 1989, MNRAS, 238, 729

Feigelson, E. D., \& Nelson, P. I., 1985, ApJ, 293, 192

Fiore, F., Matt, G., Cappi, M., Elvis, M., Leighly, K. M., Nicastro, F., Piro, L., Siemiginowska, A., \& Wilkes, B. J., 1998, MNRAS, 298, 103

Fiore, F., Matt, G., \& Nicastro, F., 1997, ApJ, 284, 731

Forster, K. 1998, PhD Thesis, Columbia University

Forster, K. \& Halpern, J. P., 1996, ApJ, 468, 565

Gelderman, R., \& Whittle, M., 1994, ApJS, 91, 491

George, I. M. \& Fabian, A. C., 1991, MNRAS, 249, 352

George, I. M., Turner, T. J., Netzer, H., Nandra, K., Mushotzky, R. F., \& Yaqoob, T., 1998, ApJS, 114, 73

Ghisellini, G., \& Haardt, F., 1994, ApJL, 429, 53

Gierliński, M., Zdziarski, A. A., Poutanen, J., Coppi, P., Ebisawa, K., \& Johnson, W. N., 1999, MNRAS, in press

Goncalves, A. C., Véron, P., \& Véron-Cetty, M.-P. 1998, in Proc. "Structure and Kinematics of Quasar Broad Line Regions", eds. C. M. Gaskell, W. N. Brandt, M. Dietrich, D. Dultzin-Hacyan \& M. Eracleous, in press

Goodrich, R. W. 1989, ApJ, 342, 224

Grupe, D. 1996, PhD Thesis, University of Göttingen

Grupe, D., Beuermann, K., Mannheim, K., Bade, N., Thomas, H.-C., De Martino, D., \& Schwope, A., 1995a, A\&A, 299, 5

Grupe, D., Beuermann, K., Mannheim, K., Thomas, H.-C., Fink, H. H., \& De Martino, D., 1995b, A\&A, 300, 21

Grupe, D., Beuermann, K., Thomas, H.-C., Mannheim, K. \& Fink, H. H. 1998a, A\&A, 330, 25

Grupe, D., Wills, B. J., Wills, D., \& Beuermann, K., 1998b, A\&A, 333, 827

Grupe, D., Beuermann, K., Mannheim, K., \& Thomas, H.-C., 1999a, A\&A, in press

Grupe, D., Leighly, K. M., Thomas, H.-C., Laurent-Muehleisen, S. A., 1999b, A\&A, submitted

Guainazzi, M., Matsuoka, M., Piro, L., Mihara, T. \& Yamauchi, M., 1994, ApJL, 436, 35

Guainazzi, M., Mihara, T., Otani, C., \& Matsuoka, M., 1996, PASJ, 48, 781 
Guainazzi, M., Piro, L., Capalbi, M., Parmar, A. N., Yamauchi, M., \& Matsuoka, M., 1998a, A\&A, 339, 337

Guainazzi, M., et al., 1998b, A\&A, 339, 327

Haardt, F., \& Maraschi, L., 1991, ApJL, 380, 51

Haardt, F., \& Maraschi, L., 1993, ApJ, 413, 507

Haardt, F., Maraschi, L., \& Ghisellini, G., 1994, ApJ, 432, 95

Halpern, J. P., \& Moran, E. C. 1998, ApJ, 494, 194

Hayashida, K. 1996, Proc. "Emission Lines in AGN: New Methods and Techniques", ed. B. M. Peterson, F.-Z. Cheng \& A. S. Wilson, (San Fransisco: ASP), 40

Isobe, T., \& Feigelson, E. D., 1990, BAAS, 22, 917

Iwasawa, K., Brandt, W. N., \& Fabian, A. C., 1998, MNRAS, 293, 251

Joly, M., 1993, Ann. Phys. Fr., 18, 241

Kay, L. E., Magalhães, A. M., Elizalde, F., Rodrigues, C., 1999, ApJ, 518, 219

Korista, K. T. 1991, AJ, 102, 41

Korista, K., Ferland, G., \& Baldwin, J., 1997, ApJ, 487, 555

Koski, A. T. 1978, ApJ, 223, 56

Krolik, J. H., \& Kallman, T. R. 1988, ApJ, 324, 714

Krolik, J. H., \& Kriss, G. A., 1995, ApJ, 447, 512

Kwan, J., Cheng, F.-Z., Fang, L.-Z., Zheng, W., \& Ge, J., 1995, ApJ, 440, 628

Laor, A., Fiore, F., Elvis, M., Wilkes, B. J., \& McDowell, J. C., 1997a, ApJ, 477, 93

Laor, A., Jannuzzi, B. T., Green, R. F., \& Boroson, T. A., 1997b, ApJ, 489, 656

Laor, A., \& Netzer, H., 1989, MNRAS, 238, 897

Lawrence, A., \& Elvis, M., 1982, ApJ, 256, 410

Lawrence, A., Elvis, M., Wilkes, B. J., McHardy, I., \& Brandt, N. 1997, MNRAS, 286, 879

Leighly, K. M. 1998, in proc. "Accretion Processes in Astrophysical Systems: Some Like it Hot!", eds. S. S. Holt, T. R. Kallman, (AIP: Woodbury, New York), p. 199

Leighly, K. M., Kay, L. E., Wills, B. J., Wills, D., \& Grupe, D. 1997b, ApJL, 489, 137

Leighly, K. M., Mushotzky, R. F., Yaqoob, T., Kunieda, K., \& Edelson, R., 1996, ApJ, 469, 14

Leighly, K. M., Mushotzky, R. F., Nandra, K., Forster, K., 1997a, ApJL, 489, 25

Leighly, K. M., \& O’Brien, P. T., 1997, ApJL, 481, 15

Lightman, A. P. \& Eardley, D. M. 1974, ApJL, 187, 1

Maccacaro, T., Gioia, I. M., Wolter, A., Zamorani, G., \& Stocke, J. T., 1988, ApJ, 326, 680 
Madau, P. 1998, ApJ, 327, 116

Makuch, R. W., Escobar, M., \& Merrill III, S., 1991, Appl. Statist. 40, 19

Mason, K. O., Puchnarewicz, E. M., \& Jones, L. R., 1996, MNRAS, 283, 26

Matt, G., Fabian, A. C., \& Ross, R. R., 1993b, MNRAS, 262, 179

Matt, G., Fabian, A. C., \& Ross, R. R. 1993a, MNRAS, 264, 839

Molendi, S., \& Maccacaro, T., 1994, A\&A, 291, 420

Moran, E. C., Halpern, J. P., \& Helfand, D. J., 1996, ApJS, 106, 341

Morrison, R., \& McCammon, D., 1983, ApJ, 270, 119

Murphy, E. M., Lockman, F. J., Laor, A., \& Elvis, M. 1996, ApJS, 105, 369

Mushotzky, R. F. 1982, ApJ, 256, 92

Mushotzky, R. F., Fabian, A. C., Iwasawa, K., Kunieda, H., Matsuoka, M., Nandra, K., \& Tanaka, Y. 1995, MNRAS, 272, 9

Nandra, K., George, I. M., Mushotzky, R. F., Turner, T. J., \& Yaqoob, T., 1997a, ApJ, 477, 602

Nandra, K., George, I. M., Mushotzky, R. F., Turner, T. J., \& Yaqoob, T., 1997b, ApJL, 488, 91

Nandra, K., \& Pounds, K. A., 1994, MNRAS, 267, 974

Narayan, R., Mahadevan, R., \& Quataert, E., 1999, in "Theory of Black Hole Accretion Disks", eds. M. A. Abramowicz, G. Bjornsson, \& J. E. Pringle, in press

Netzer, H., 1996, ApJ, 473, 781

Nicastro, F., Fiore, F., Perola, G. C., \& Elvis, M., 1999, ApJ, 512, 184

Nicastro, F., Fiore, F., \& Matt, G., 1999, ApJ, 517, 108

Nishimura, J., Mitsuda, K, \& Itoh, M., 1986, PASJ, 38, 819

Nowak, M. A. 1995, PASP, 107, 1207

Osterbrock, D. E., \& Pogge, R. W. 1985, ApJ, 297, 166

Otani, C., Kii, T. \& Miya, K. 1996, Proc. "Röntgenstrahlung from the Universe", eds. Zimmermann, H. U., Trümper, J., and Yorke H., 1996, MPE Report 263, 491

Otani, C. et al. 1996, PASJ, 48, 211

Phillips, M. M., 1976, ApJ, 208, 37

Phlips, B. F. et al., 1996, ApJ, 465, 907

Piccinotti, G., Mushotzky, R. F., Boldt, E. A., Holt, S. S., Marshall, F. E., Serlemitsos, P. J., \& Shafer, R. A., 1982, ApJ, 253, 485

Pietrini, P., \& Krolik, J. H. 1995, ApJ, 447, 526

Pogge, R. W., \& Owen, J. M. 1993, OSU Internal Report 93-01 
Porquet, D., Dumont, A.-M., Collin, S., \& Mouchet, M., 1999, A\&A, 341, 58

Pounds, K. A., Done, C., \& Osborne, J., 1996, MNRAS, 277, L5

Press, W. H., Teukolsky, S. A., Vettering, W. T., \& Flannery, B. P. 1992, Numerical Recipes, (Cambridge University Press: Cambridge)

Puchnarewicz, E. M. et al. 1992, MNRAS, 256, 589

Remillard, R. A., Bradt, H. V., Buckley, D. A. H., Roberts, W., Schwartz, D. A., Tuohy, I. R., \& Wood, K. 1986, ApJ, 301, 742

Reynolds, C. S., 1997, MNRAS, 286, 513

Reynolds, C. S., \& Fabian, A. C., 1995, MNRAS, 273, 1167

Reynolds, C. S., Ward, M. J., Fabian, A. C., \& Celotti, A. 1997, MNRAS, 291, 403

Ross, R. R., Fabian, A. C., \& Mineshige, S., 1992, MNRAS, 258, 189

Rybicki, G. B., \& Lightman, A. P., 1979, "Radiative Processes in Astrophysics" (Wiley: New York)

Schartel, N., Walter, R., Fink, H. H., \& Trümper, J., 1996, A\&A, 307, 33

Shakura, N. I., \& Sunyaev, R. A., 1973, A\&A, 24, 337

Shapiro, S. L., Lightman, A. P., \& Eardley, D. M., 1976, ApJ, 204, 187

Shields, J. C., Ferland, G. J., \& Peterson, B. M., 1995, ApJ, 441, 507

Shimura, T., \& Takahara, F., 1993, ApJ, 419, 78

Shrader, C., \& Titarchuk, L., 1998, ApJL, 499, 31

Smith, P. S., Schmidt, G. D., Allen, R. G. \& Hines, D. C. 1998, ApJ, 448, 202

Stern, B. E., Poutanen, J., \& Svensson, R. 1995, ApJL, 449, 13

Swensson, R., 1994, ApJS, 92, 585

Svensson, R., \& Zdziarski, A. A., 1994, ApJ, 436, 599

Szuszkiewicz, E., Malkan, M. A., \& Abramowicz, M. A., 1996, ApJ, 458, 474.

Tanaka, Y., et al. 1995, Nature, 375, 659

Turner, T. J., George, I. M., \& Nandra, K., 1998, ApJ, 508, 648

Turner, T. J., \& Pounds, K. A., 1989, MNRAS, 240, 833

Wandel, A., \& Boller, T., 1998, A\&A, 331, 884

Wilkes, B. J., Elvis, M. \& McHardy, I. 1987, ApJ, 321, 23

Wills, B. J., \& Brotherton, M. S., 1995, ApJL, 448, 81

Wills, B., J., Laor, A., Brotherton, M. S., Wills, D., Wilkes, B. J., Ferland, G. J., \& Shang, Z., 1999, ApJL, 515, 53 
Wills, B. J., Wills, D., Evans, N. J., Natta, A., Thompson, K. L., Breger, M., \& Sitko, M. L. 1992, ApJ, 400, 96

Yaqoob, T., 1998, ApJ, 500, 893

Zheng, W., Kriss, G. A., \& Davidsen, A. F. 1995, ApJ, 440, 606

Zheng, W., \& O'Brien, P. T. 1990, ApJ, 353, 433

Życki, P. T., Krolik, J. H., Zdziarski, A. A., \& Kallman, T. R., 1994, ApJ, 437, 597 
Table 1. Target Properties and Optical Emission Line Parameters

\begin{tabular}{|c|c|c|c|c|c|c|c|}
\hline Target & $\mathrm{z}$ & $\begin{array}{l}\mathrm{H} \beta \text { FWHM } \\
(\mathrm{km} / \mathrm{s})\end{array}$ & $\begin{array}{l}\mathrm{H} \beta \text { Eq. W. } \\
(\AA)\end{array}$ & $\mathrm{Fe} \mathrm{II} / \mathrm{H} \beta$ & $\begin{array}{l}\text { [O III] FWHM } \\
(\mathrm{km} / \mathrm{s})\end{array}$ & {$[\mathrm{O}$ III $] / \mathrm{H} \beta$} & Reference \\
\hline Mrk 335 & 0.025 & 1640 & 95 & 0.62 & & 0.23 & BG92 \\
\hline I Z $\mathrm{Zw} 1$ & 0.061 & 1240 & 51 & 1.47 & & 0.43 & BG92 \\
\hline Ton S180 & 0.062 & 980 & 45 & 0.8 & 640 & 0.12 & G96 \\
\hline PHL 1092 & 0.396 & 1790 & $63^{1}$ & 1.8 & & 0.91 & BK80 LEWMB97 this paper \\
\hline RX J0439-45 & 0.224 & 1010 & 65 & 0.55 & 1020 & 0.17 & G96 \\
\hline NAB $0205+024$ & 0.155 & 1050 & 58 & 0.62 & & 0.36 & ZO90 GW94 BK84 K91 \\
\hline PKS 0558-504 & 0.137 & 1250 & 45 & $1.56^{2}$ & & 0.04 & C97 R86 \\
\hline $1 \mathrm{H} 0707-495$ & 0.0411 & 1050 & 32 & 1.36 & 1516 & 0.19 & this paper \\
\hline Mrk 142 & 0.04494 & 1470 & 67 & 1.11 & 404 & 0.16 & this paper; G96 \\
\hline RE $1034+39$ & 0.04244 & 840 & 20 & 0.32 & 543 & 1.25 & this paper MPJ96 GVV98 \\
\hline NGC 4051 & 0.00242 & 1150 & 28 & 0.77 & 325 & 0.92 & this paper \\
\hline PG $1211+143$ & 0.0809 & 1860 & 84 & 0.52 & & 0.14 & BG92 \\
\hline Mrk 766 & 0.01293 & $850^{3}$ & 71 & 0.52 & 360 & 1.85 & OP85 G89 GWWB98 \\
\hline PG $1244+026$ & 0.048 & 830 & 41 & 1.20 & & 0.41 & BG92 \\
\hline IRAS $13224-3809$ & 0.0667 & 650 & 23 & 2.42 & 810 & 0.60 & this paper; K98 \\
\hline IRAS $13349+2438$ & 0.108 & 2200 & 72 & $6.5^{4}$ & & 0.13 & GWWB98 \\
\hline PG $1404+226$ & 0.098 & 880 & 54 & 1.01 & & 0.12 & BG92 \\
\hline Mrk 478 & 0.079 & 1450 & 64 & 1.19 & & 0.15 & BG92 \\
\hline IRAS $17020+4544$ & 0.0604 & 1040 & 23 & 1.86 & 1021 & 2.48 & this paper; LKWWG97 \\
\hline Mrk 507 & 0.055 & 1150 & 19 & 1.45 & 454 & 0.43 & this paper \\
\hline KAZ 163 & 0.063 & 1620 & 76 & 0.57 & 534 & 0.86 & this paper \\
\hline IRAS 20181-2244 & 0.185 & 370 & 22 & 0.89 & 537 & 6.12 & this paper; K98; HM98 \\
\hline Ark 564 & 0.024 & 950 & 43 & 0.95 & 350 & 0.96 & this paper \\
\hline
\end{tabular}

${ }^{1}$ This number is very uncertain and likely to be an overestimate because of possible Fe II emission in the vicinity of $\mathrm{H} \beta$.

${ }^{2}$ Total red and blue complexes, taken from Remillard et al. 1986, apparently measured between $4434 \AA$ and $4684 \AA$ (the usual for the blue component) and $5147 \AA$ and $5350 \AA$ for the red component. For correlations I used 0.94 , as $60 \%$ of the Fe II in the above ranges is in the blue component in the I $\mathrm{Zw} 1$ template.

${ }^{3}$ The width of the so-called narrow component of $\mathrm{H} \beta$ listed by Osterbrock \& Pogge 1985. This corresponds most closely to the FWHM measured from Figure 4 of Goodrich 1989.

${ }^{4}$ Grupe et al. $1998 \mathrm{~b}$ report the equivalent width of the whole Fe II complex from $4250 \AA$ to $5880 \AA$ found using the I Zw 1 template. I use $30 \%$ of this value for the correlations.

References. - BG92: Boroson \& Green 1992; G96: Grupe 1996; BK80: Bergeron \& Kunth 1980; LEWMB97: Lawrence et al. 1997; ZO90: Zheng \& O'Brien 1990; GW94: Gelderman \& Whittle 1994; BK84: Bergeron \& Kunth 1984; K91: Korista 1991; C97: Corbin 1997; R86: Remillard et al. 1986; MPG96: Mason, Puchnarewicz \& Jones 1996; GVV98: Goncalves, Veron \& Veron-Cetty 1998; OP85: Osterbrock \& Pogge 1985; G89: Goodrich 1989; GWWB98: Grupe et al. 1998b; K98: Kay et al. 1998; LKWWG97: Leighly et al. 1997b; HM98: Halpern \& Moran 1998. 
Table 2. Preliminary Spectral Fitting Results

\begin{tabular}{lccccc}
\hline \hline \multicolumn{1}{c}{ Target } & d.o.f. ${ }^{1}$ & $\begin{array}{c}\text { Power Law } \\
\chi_{\nu}^{2}\end{array}$ & $\begin{array}{c}\text { Soft Excess } \\
\chi_{\nu}^{2}\end{array}$ & $\begin{array}{c}\text { Two-Edge } \\
\chi_{\nu}^{2}\end{array}$ & $\begin{array}{c}\text { Absori }{ }^{2} \\
\chi_{\nu}^{2}\end{array}$ \\
& & & & & \\
\hline Mrk 335 & 739 & 969 & 749 & 939 & 786 \\
I Zw 1 & 549 & 627 & 627 & 626 & 623 \\
Ton S180 & 856 & 1109 & 888 & 1109 & 1011 \\
PHL 1092 & 246 & 335 & 239 & 335 & 265 \\
RX J0439-45 & 330 & 475 & 320 & 475 & 341 \\
NAB 0205+024 & 599 & 638 & 602 & 638 & 612 \\
PKS 0558-504 & 1093 & 1126 & 1087 & 1126 & 1113 \\
1H 0707-495 & 368 & 955 & 531 & 897 & 623 \\
Mrk 142 & 365 & 364 & 336 & 364 & 345 \\
RE 1034+39 & 324 & 415 & 315 & 400 & 330 \\
NGC 4051(1) & 1177 & 2759 & 1313 & 2329 & 1698 \\
NGC 4051(2) & 1850 & 4191 & 2288 & 3716 & 2646 \\
PG 1211+143 & 582 & 1047 & 544 & 903 & 625 \\
Mrk 766 & 1385 & 2102 & 1506 & 1535 & 1425 \\
PG 1244+026 & 480 & 560 & 512 & 560 & 552 \\
IRAS 13224-3809 & 404 & 1207 & 509 & 1207 & 1207 \\
IRAS 13349+2438 & 568 & 583 & 569 & 541 & 549 \\
PG 1404+226 & 230 & 565 & 276 & 565 & 371 \\
Mrk 478 & 530 & 770 & 590 & 677 & 620 \\
IRAS 17020+4544 & 939 & 1347 & 1347 & 1327 & 1080 \\
Mrk 507 & 152 & 150 & 150 & 142 & 137 \\
KAZ 163 & 249 & 221 & 212 & 220 & 218 \\
IRAS 20181-2244 & 400 & 663 & 663 & 656 & 456 \\
Ark 564 & 1402 & 2185 & 1590 & 2185 & 1819 \\
\hline
\end{tabular}

${ }^{1}$ Number of degrees of freedom for the power law plus Galactic absorption model. The number of degrees of freedom for the other continuum models are these numbers less 2 .

${ }^{2}$ See text for description of the models. 
Table 4. Absori Warm Absorber Spectral Fitting Results

\begin{tabular}{|c|c|c|c|c|c|c|}
\hline Target & Photon Index & $\begin{array}{c}N_{W} \\
\left(\times 10^{22} \mathrm{~cm}^{-2}\right)\end{array}$ & $\xi$ & $\begin{array}{l}\mathrm{T}^{1} \\
(\mathrm{~K})\end{array}$ & $\Delta \chi^{22}$ & $\chi^{2} /$ dof \\
\hline Mrk 335 & $2.08 \pm 0.03$ & $2.6_{-1.2}^{+2.3}$ & $570_{-250}^{+440}$ & $1.0 \times 10^{6}$ & 19 & $705 / 732$ \\
\hline I Zw 1 & $2.42_{-0.08}^{+0.07}$ & $0.06 \pm 0.04$ & $0.03_{-0.03}^{+0.24}$ & $3.0 \times 10^{4}$ & 10 & $598 / 544$ \\
\hline NAB $0205+024$ & $2.24 \pm 0.07$ & $0.28_{-0.18}^{+0.32}$ & $33_{-33}^{+120}$ & $3.0 \times 10^{4}$ & $8(\mathrm{~m})$ & $594 / 595$ \\
\hline NGC 4051(1) & $2.05_{-0.03}^{+0.04}$ & $0.11_{-0.05}^{+0.11}$ & $\begin{array}{l}1.9_{-0.9}^{+16} \\
\end{array}$ & $1.0 \times 10^{6}$ & 15 & $1243 / 1167$ \\
\hline NGC 4051(2) & $2.00 \pm 0.02$ & $0.21 \pm 0.04$ & $1.7_{-0.4}^{+0.6}$ & $1.0 \times 10^{6}$ & 90 & $2058 / 1844$ \\
\hline Mrk 766 & $1.98 \pm 0.03$ & $0.30_{-0.06}^{+0.08}$ & $9.8_{-4.0}^{+7.9}$ & $3.0 \times 10^{4}$ & 154 & $1338 / 1378$ \\
\hline PG $1244+026$ & $2.40_{-0.13}^{+0.12}$ & $0.82_{-0.56}^{+0.88}$ & $260_{-210}^{+680}$ & $3.0 \times 10^{4}$ & $6(\mathrm{~m})$ & $483 / 471$ \\
\hline IRAS $13224-3809$ & $2.07_{-0.30}^{+0.27}$ & $1.12_{-0.51}^{+0.48}$ & $86_{-35}^{+104}$ & $3.0 \times 10^{4}$ & $6(\mathrm{~m})$ & $401 / 396$ \\
\hline IRAS $13349+2438$ & $2.39 \pm 0.05$ & $0.18_{-0.05}^{+0.07}$ & $\begin{array}{l}-35.7 \\
2.1_{-1.1}^{+2.7}\end{array}$ & $1.0 \times 10^{6}$ & 38 & $540 / 563$ \\
\hline Mrk 478 & $2.22_{-0.13}^{+0.16}$ & $0.54_{-0.28}^{+0.38}$ & $21_{-16}^{+27.1}$ & $1.0 \times 10^{6}$ & 14 & $567 / 523$ \\
\hline IRAS $17020+4544$ & $2.45 \pm 0.05$ & $0.22_{-0.05}^{+0.06}$ & $24_{-15}^{+24}$ & $3.0 \times 10^{4}$ & 80 & $1043 / 935$ \\
\hline KAZ 163 & $1.50_{-0.30}^{+0.28}$ & $1.2_{-0.7}^{+0.8}$ & $60_{-40}^{+130}$ & $3.0 \times 10^{4}$ & $7(\mathrm{~m})$ & $205 / 245$ \\
\hline IRAS $20181-2244$ & $2.55_{-0.11}^{+0.12}$ & $0.24_{-0.16}^{+0.21}$ & $60_{-60}^{+290}$ & $3.0 \times 10^{4}$ & $7(\mathrm{~m})$ & $449 / 395$ \\
\hline Ark 564 & $2.59_{-0.02}^{+0.01}$ & $1.0 \pm 0.4$ & $750_{-160}^{+280}$ & $1.0 \times 10^{6}$ & 10 & $1439 / 1393$ \\
\hline
\end{tabular}

${ }^{1}$ The temperature of the ionized gas was fixed at two different values for the spectral fitting; the one that gave the better fit is listed.

${ }^{2}$ Increase in $\chi^{2}$ when the warm absorber was removed from the model. 

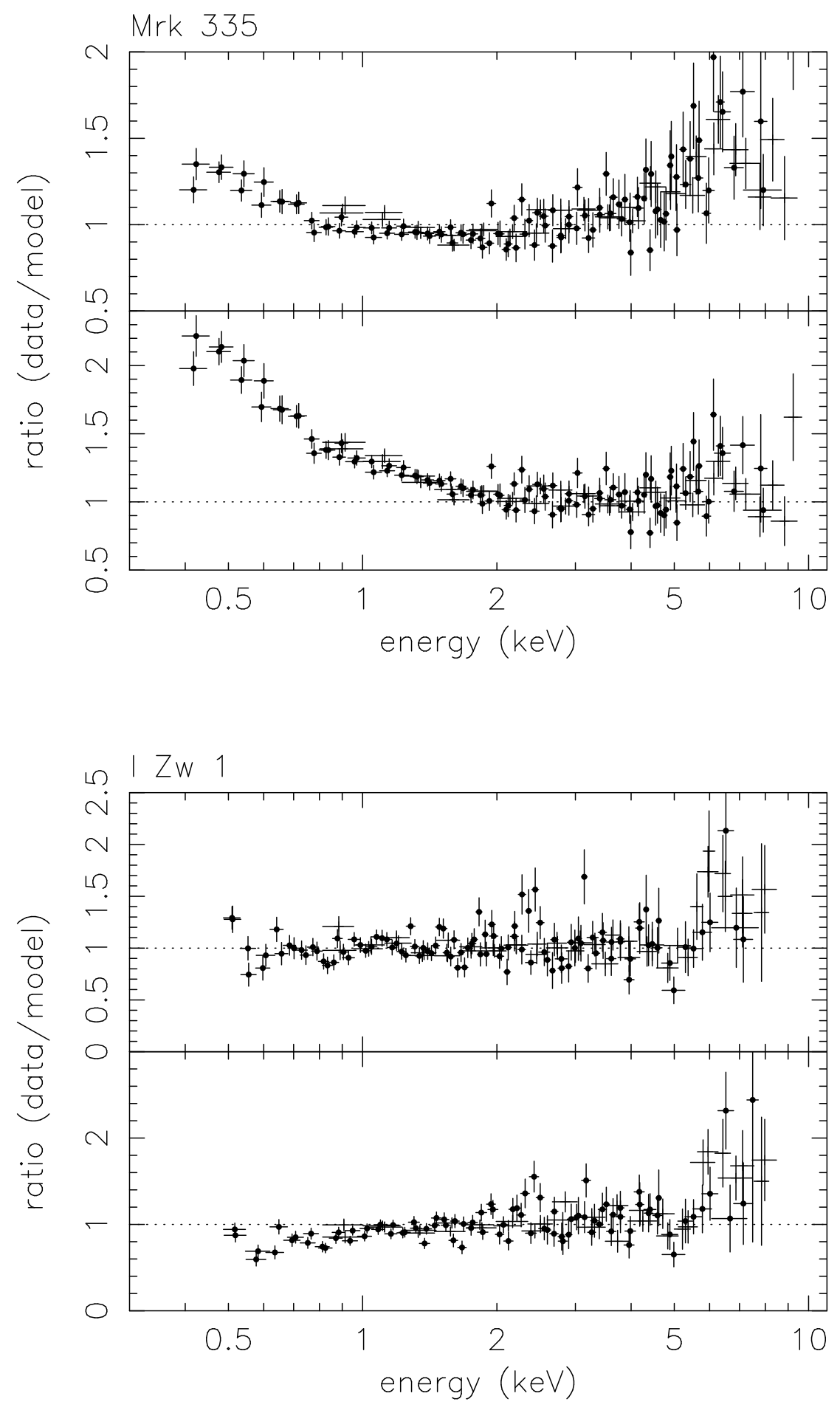

Fig. 1. - Top: Ratio of data to power law plus Galactic absorption model. Bottom: Ratio of data to best fitting power law model above $2 \mathrm{keV}$. 

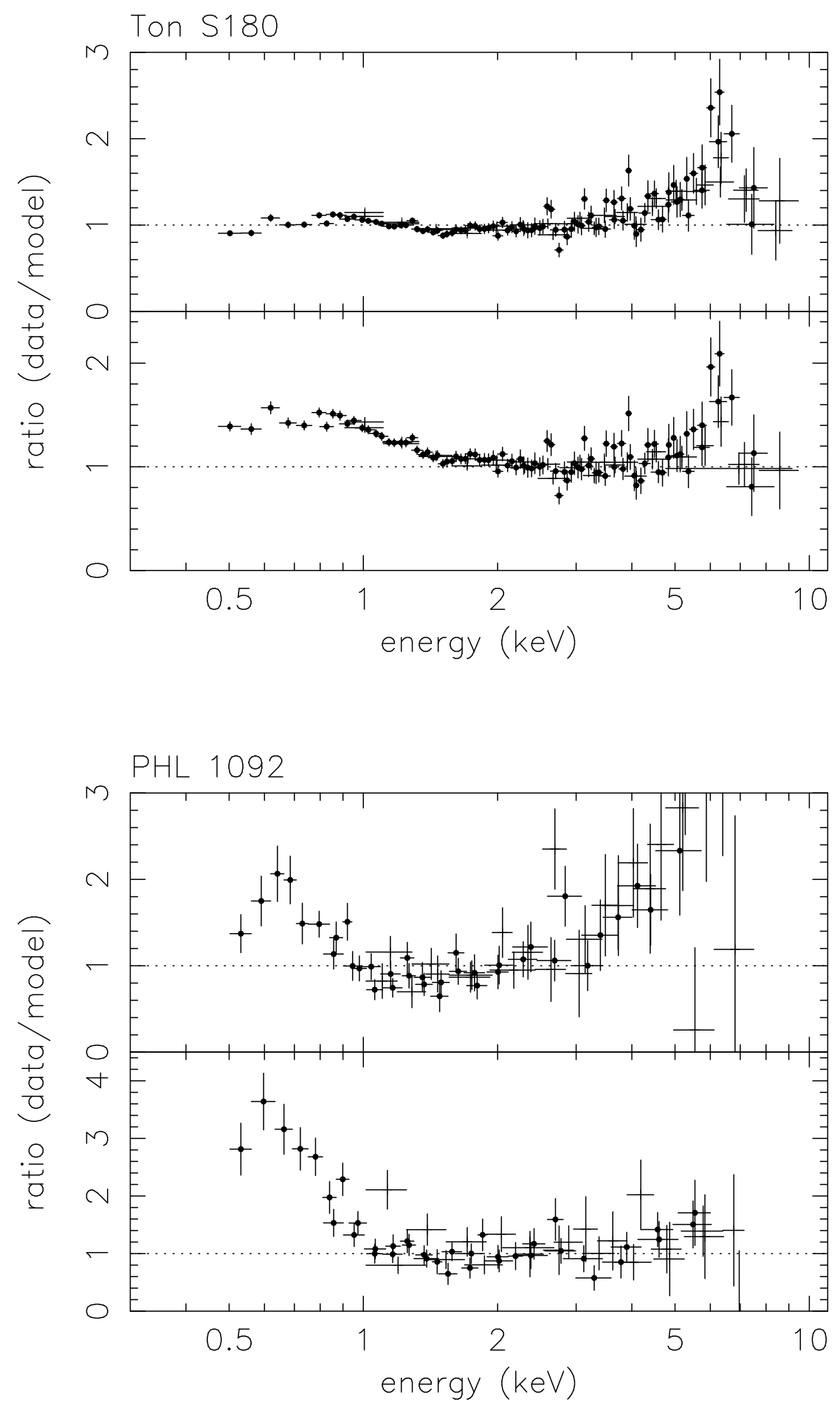

Fig. 1.- continued. 

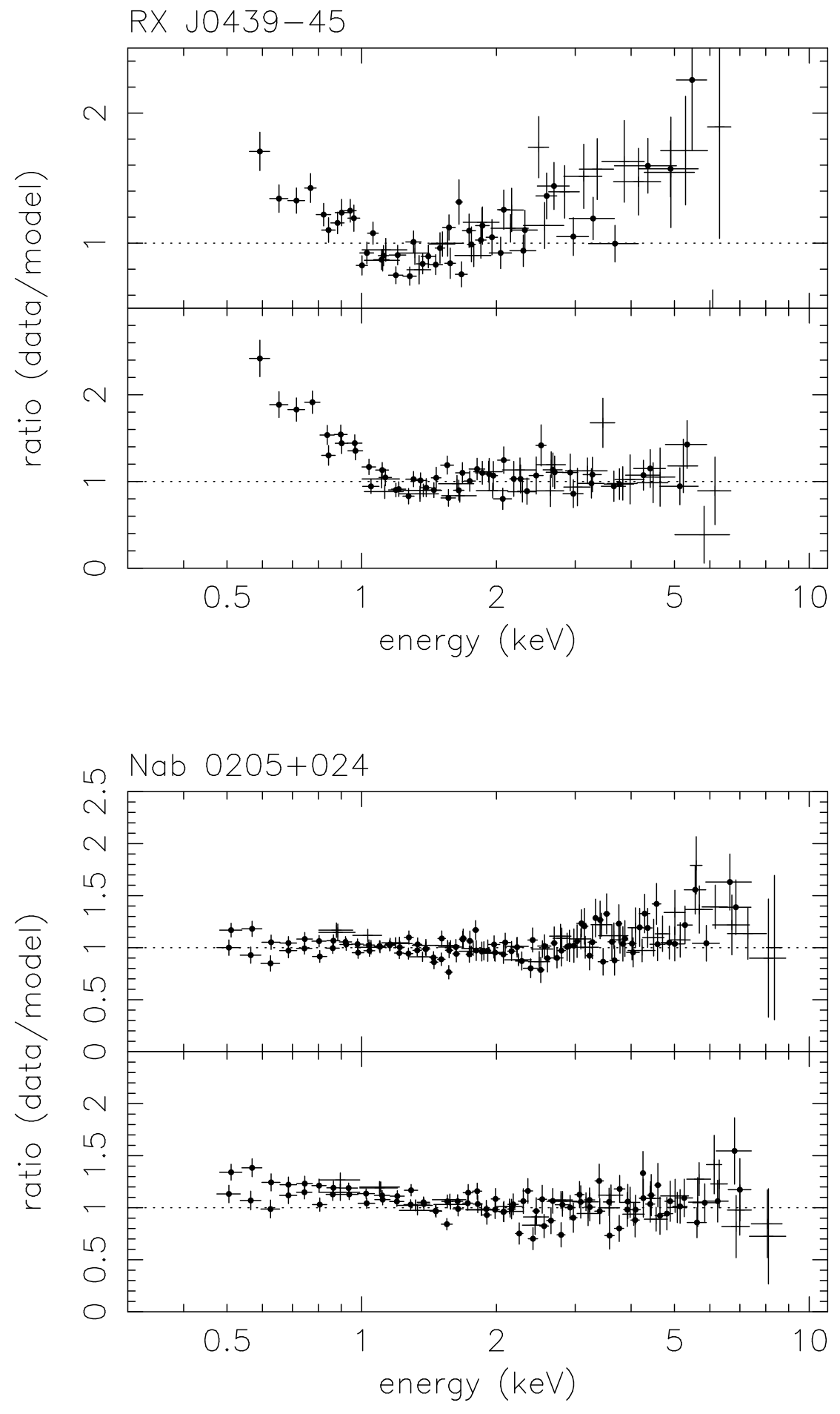

Fig. 1.- continued. 

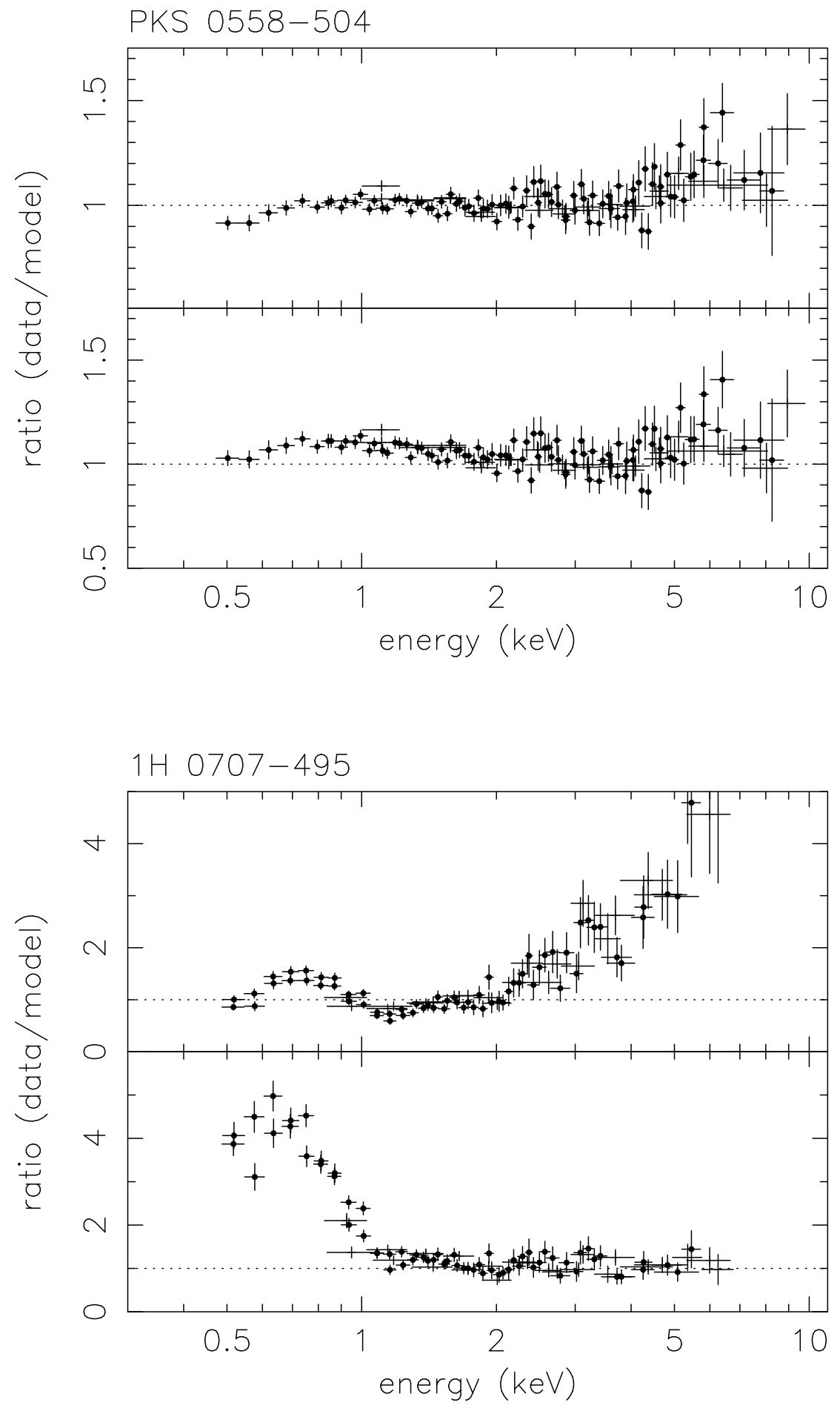

Fig. 1.- continued. 

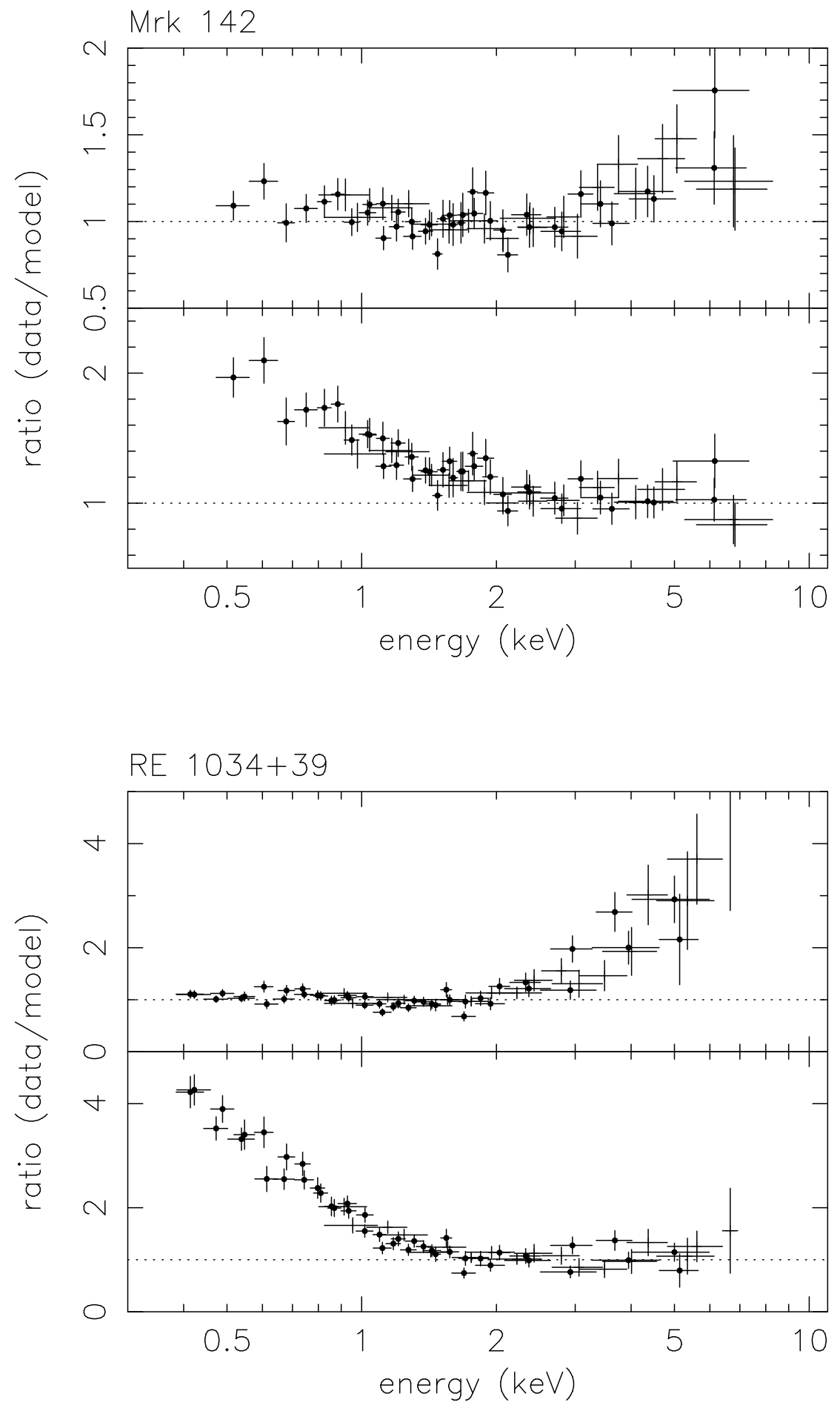

Fig. 1.- continued. 

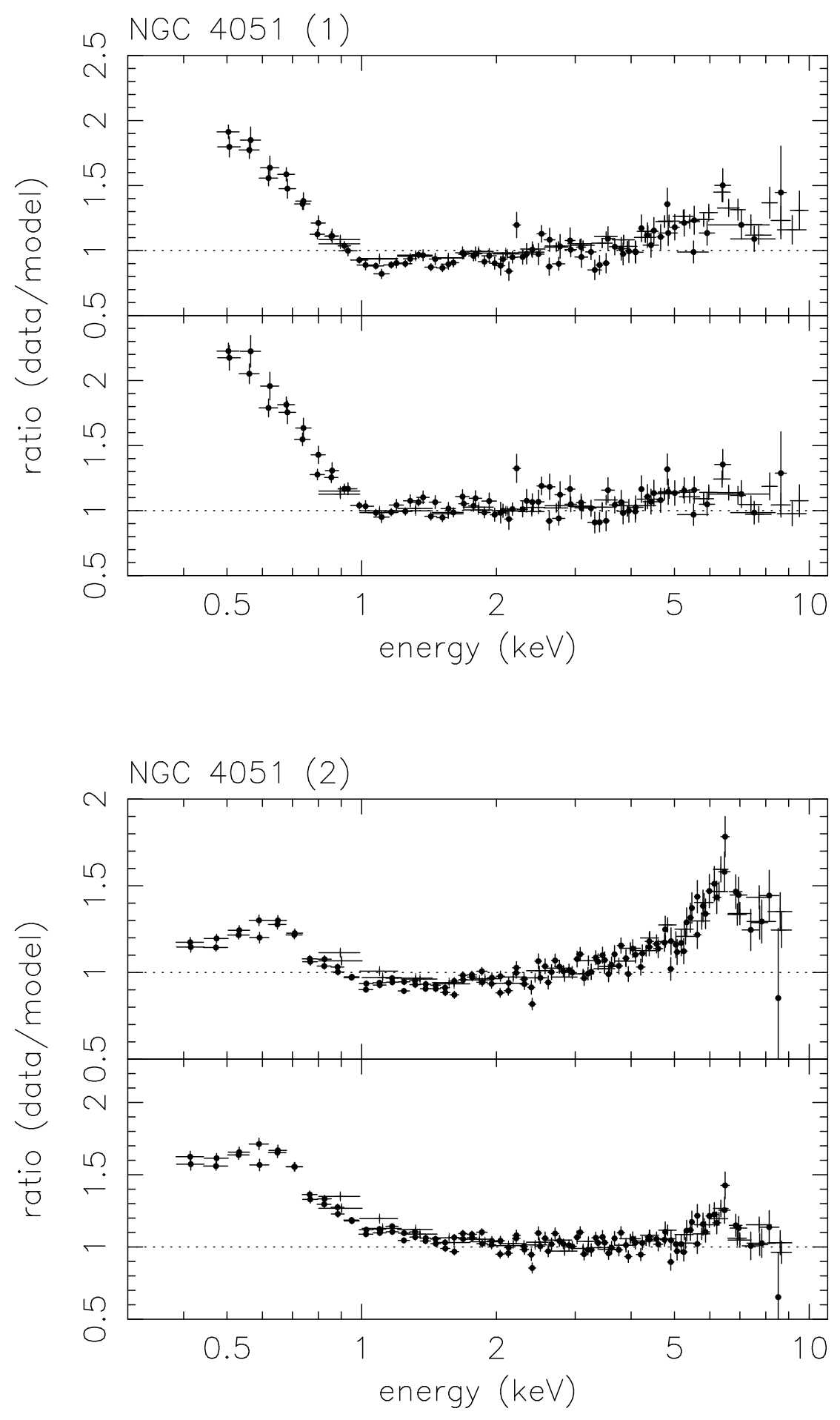

Fig. 1.- continued. 

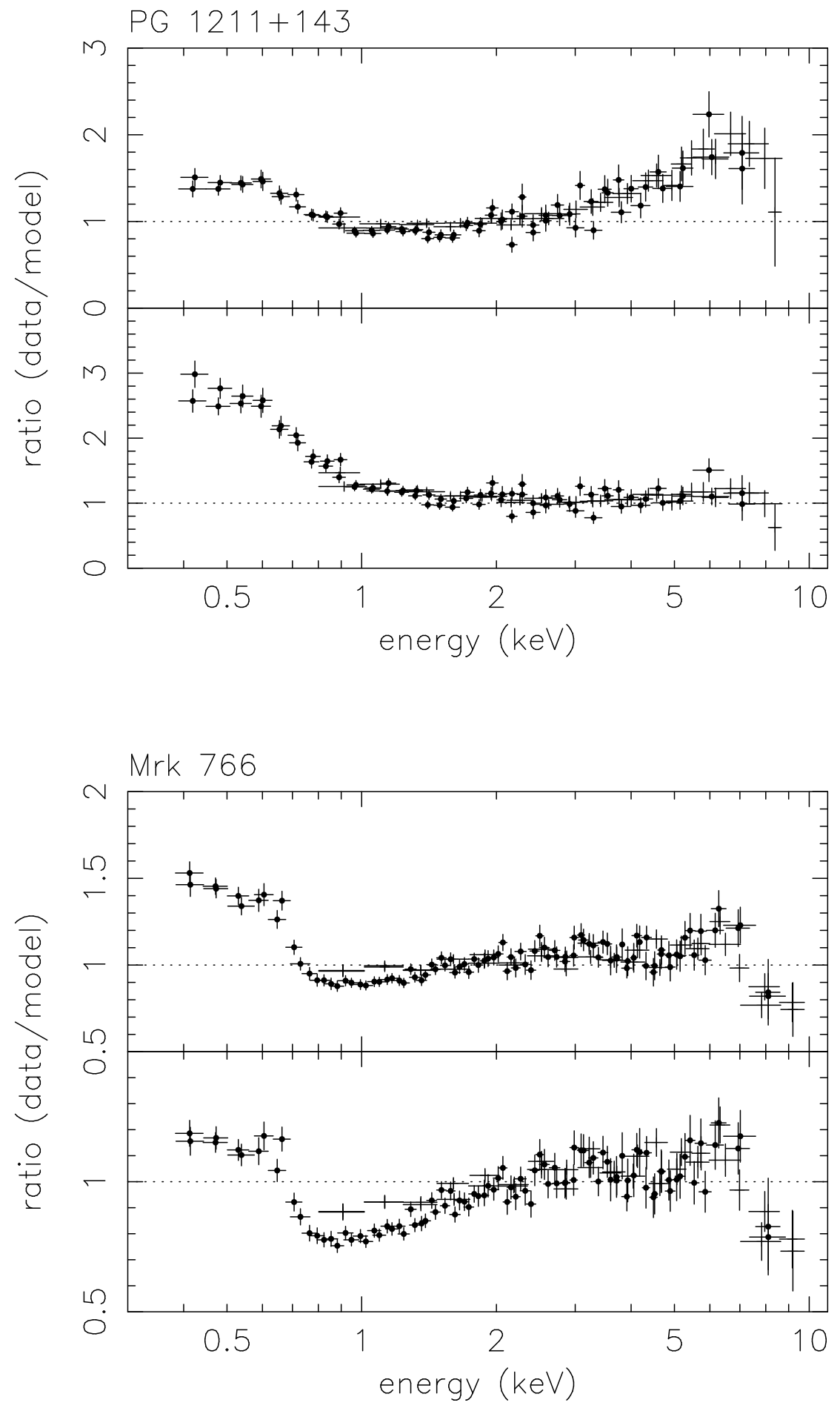

Fig. 1.- continued. 

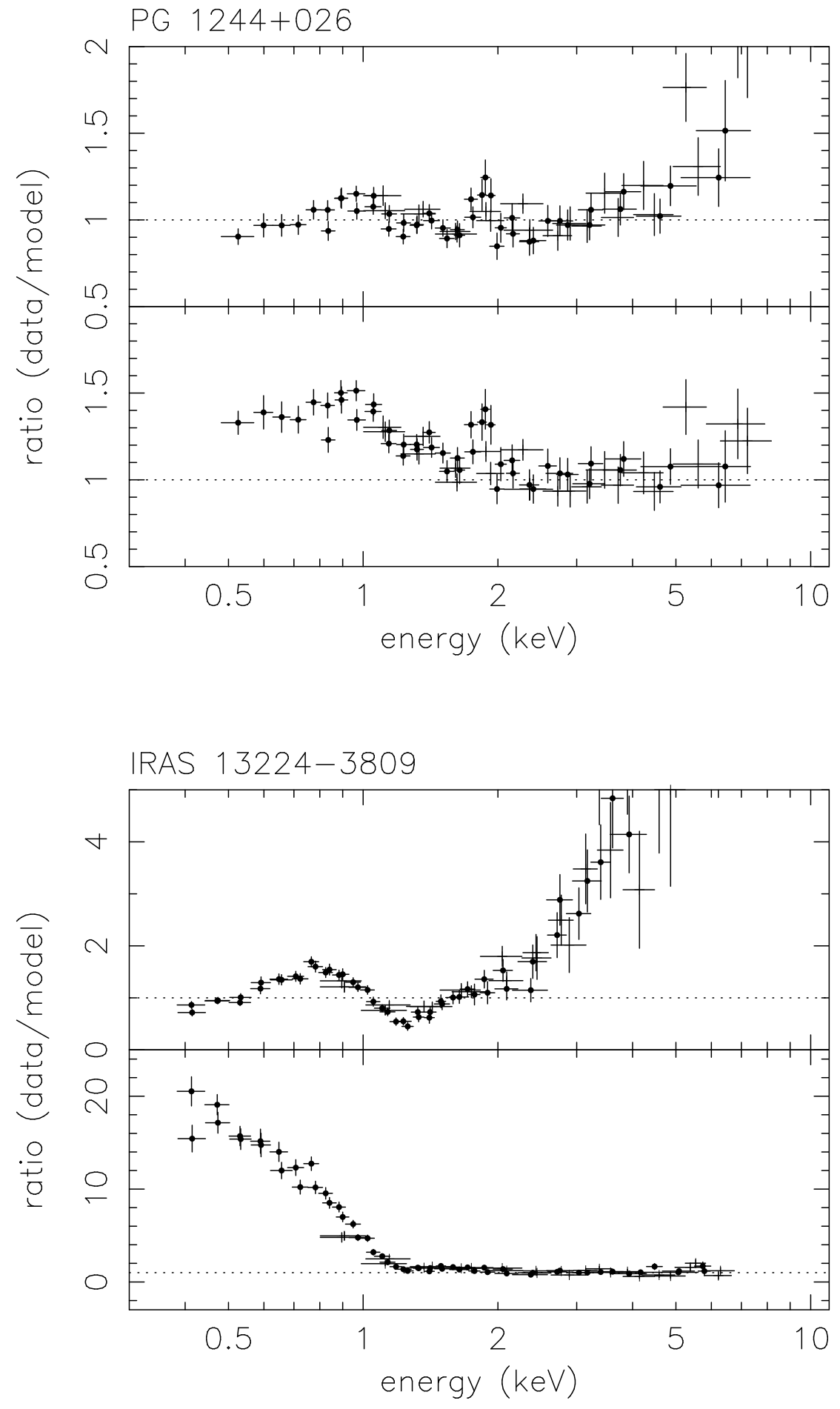

Fig. 1.- continued. 

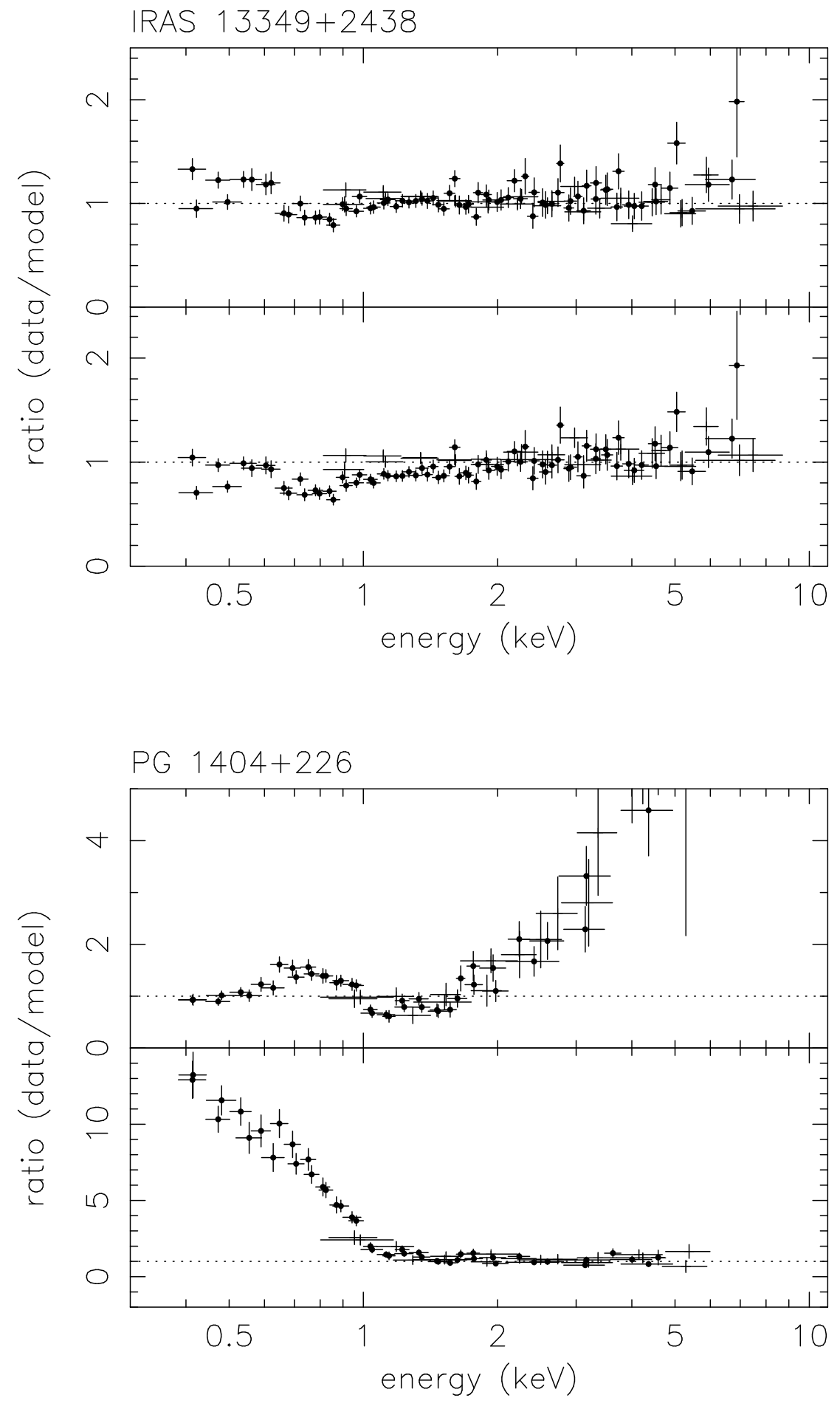

Fig. 1.- continued. 

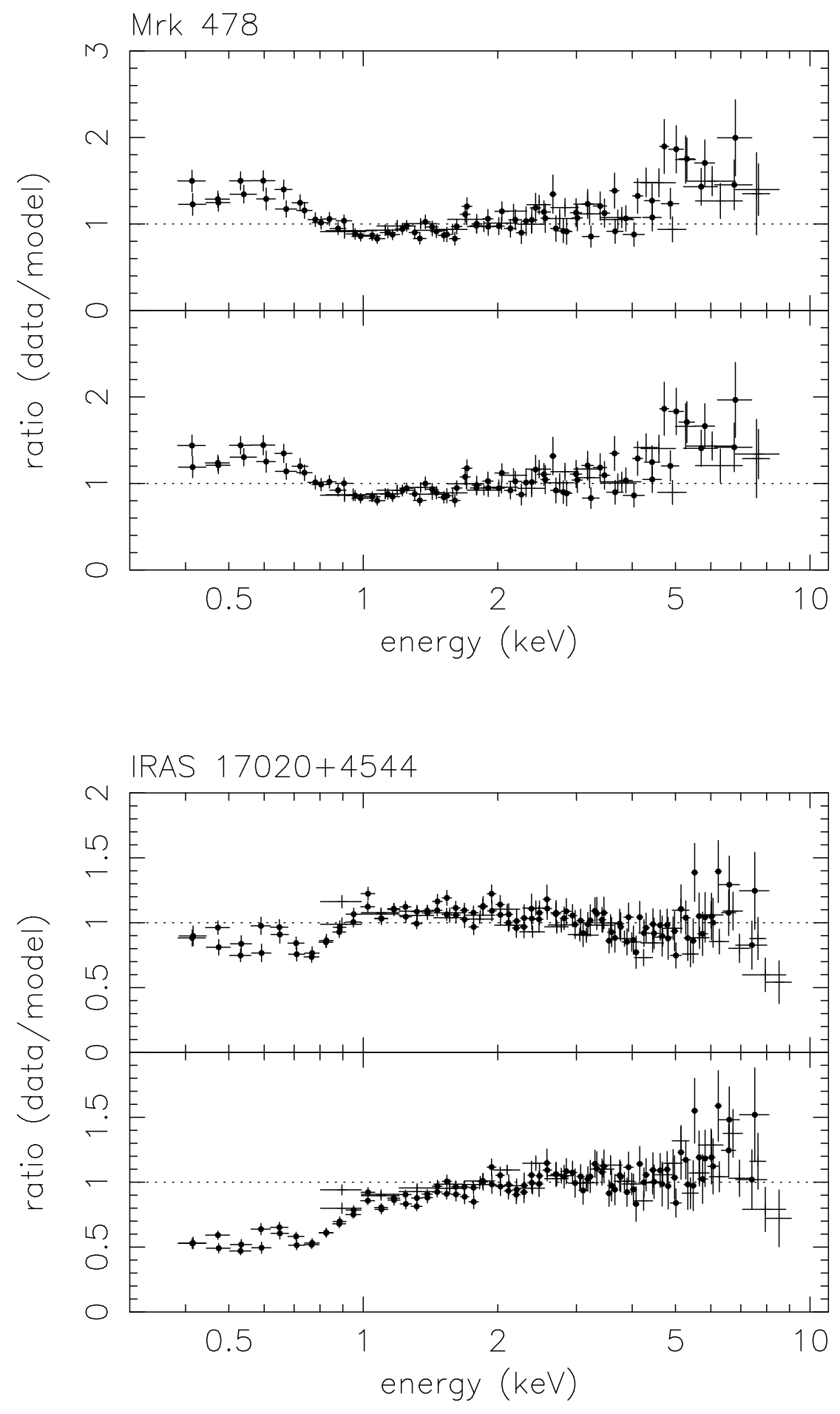

Fig. 1.- continued. 

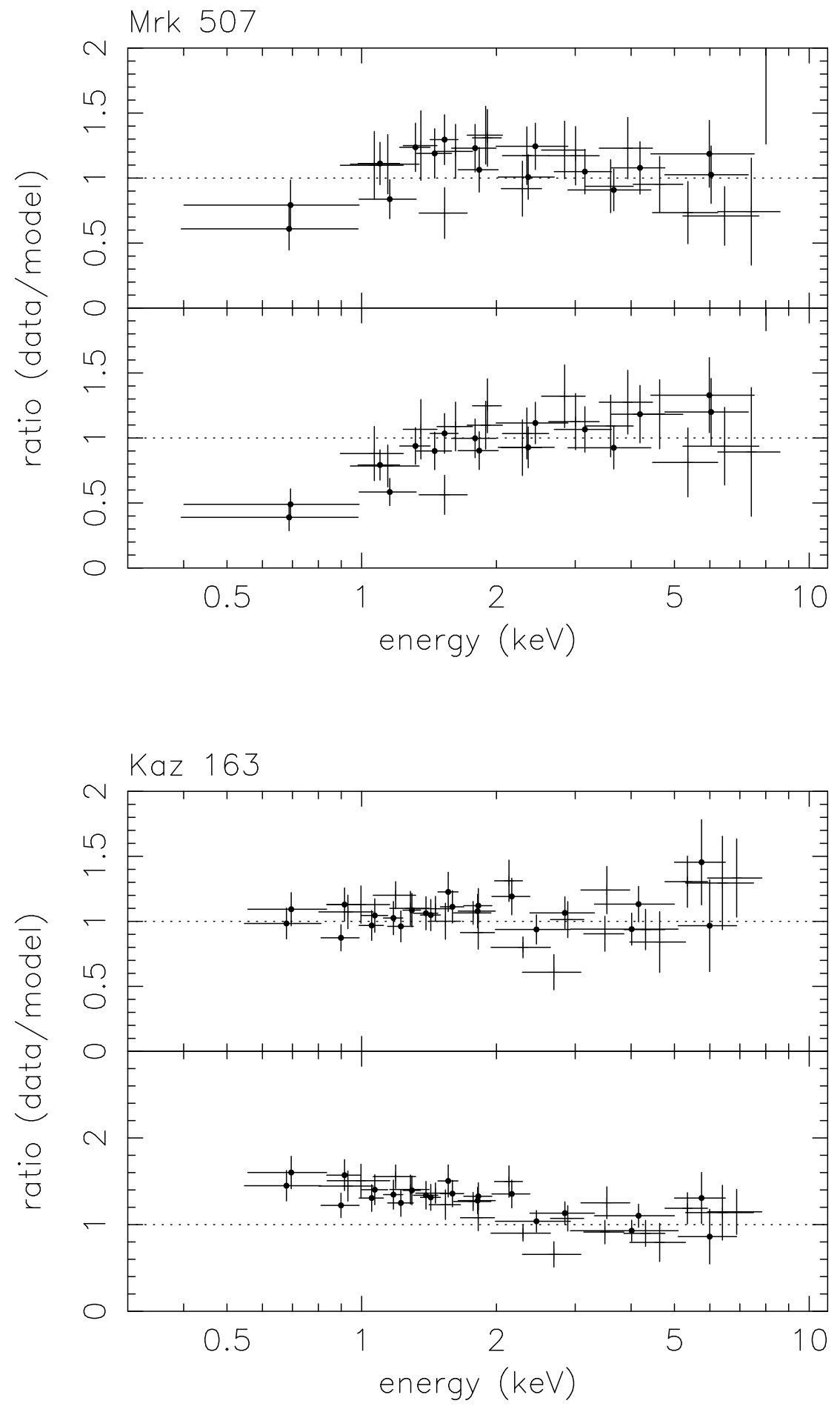

Fig. 1.- continued. 

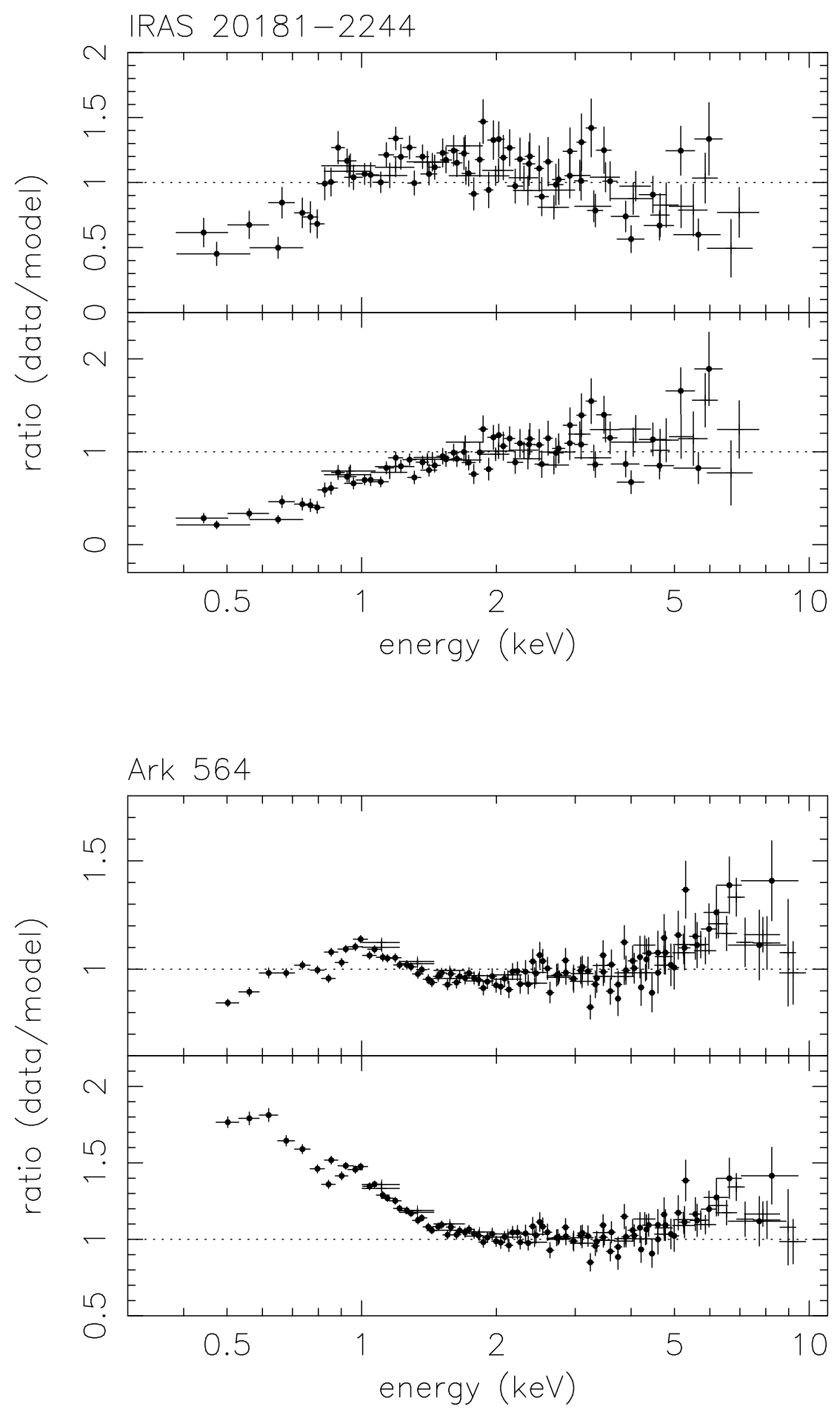

Fig. 1.- continued. 


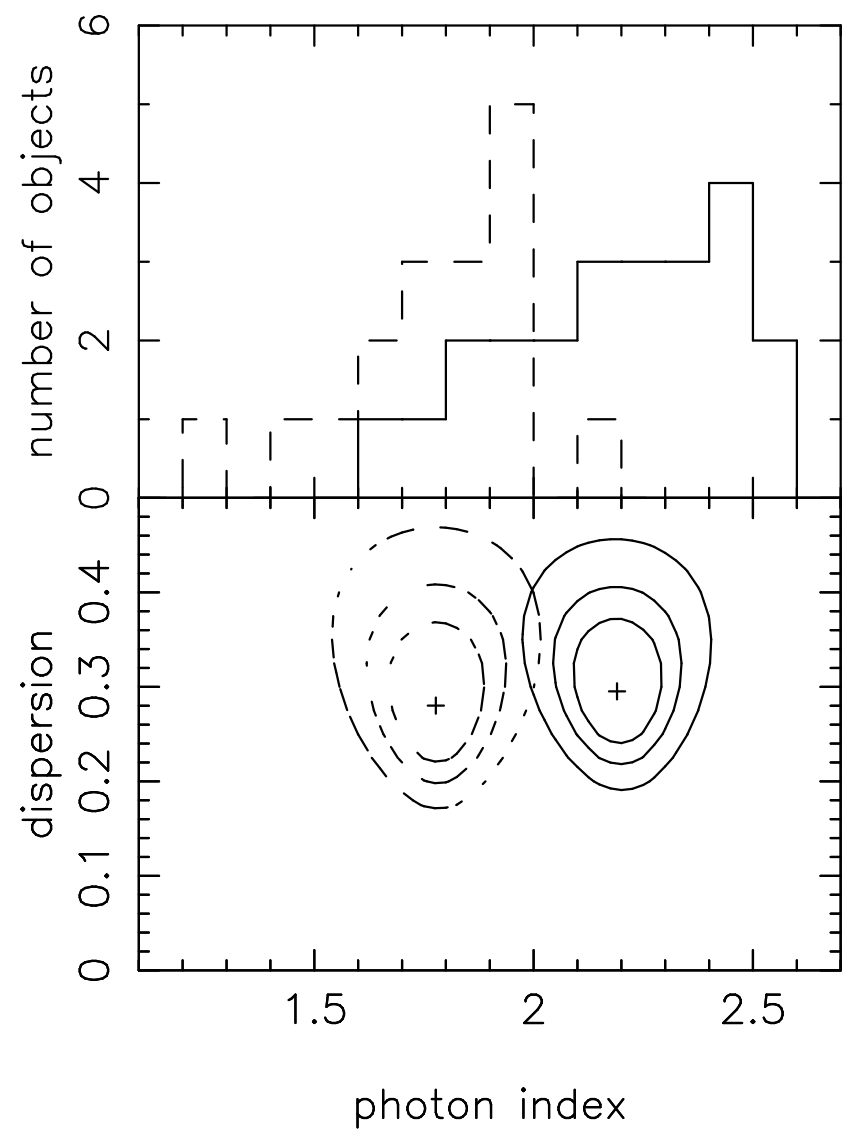

Fig. 2. - The upper panel shows the histogram of the photon indices from the 23 narrow-line Seyfert 1 galaxies analyzed in this paper (solid line) and from the 17 radio-quiet broad-line Seyfert 1 galaxies in Reynolds (1997) (dashed line). The lower panel shows the 68,90 and $99 \% \chi^{2}$ contours for two degrees of freedom from the maximum likelihood determination of the average photon index and the dispersion of the photon indices. For both broad and narrow-line objects, the dispersion is significantly greater than zero, ruling out a single photon index for each class of object, and the average photon index for the two classes are significantly different at $90 \%$ confidence. 


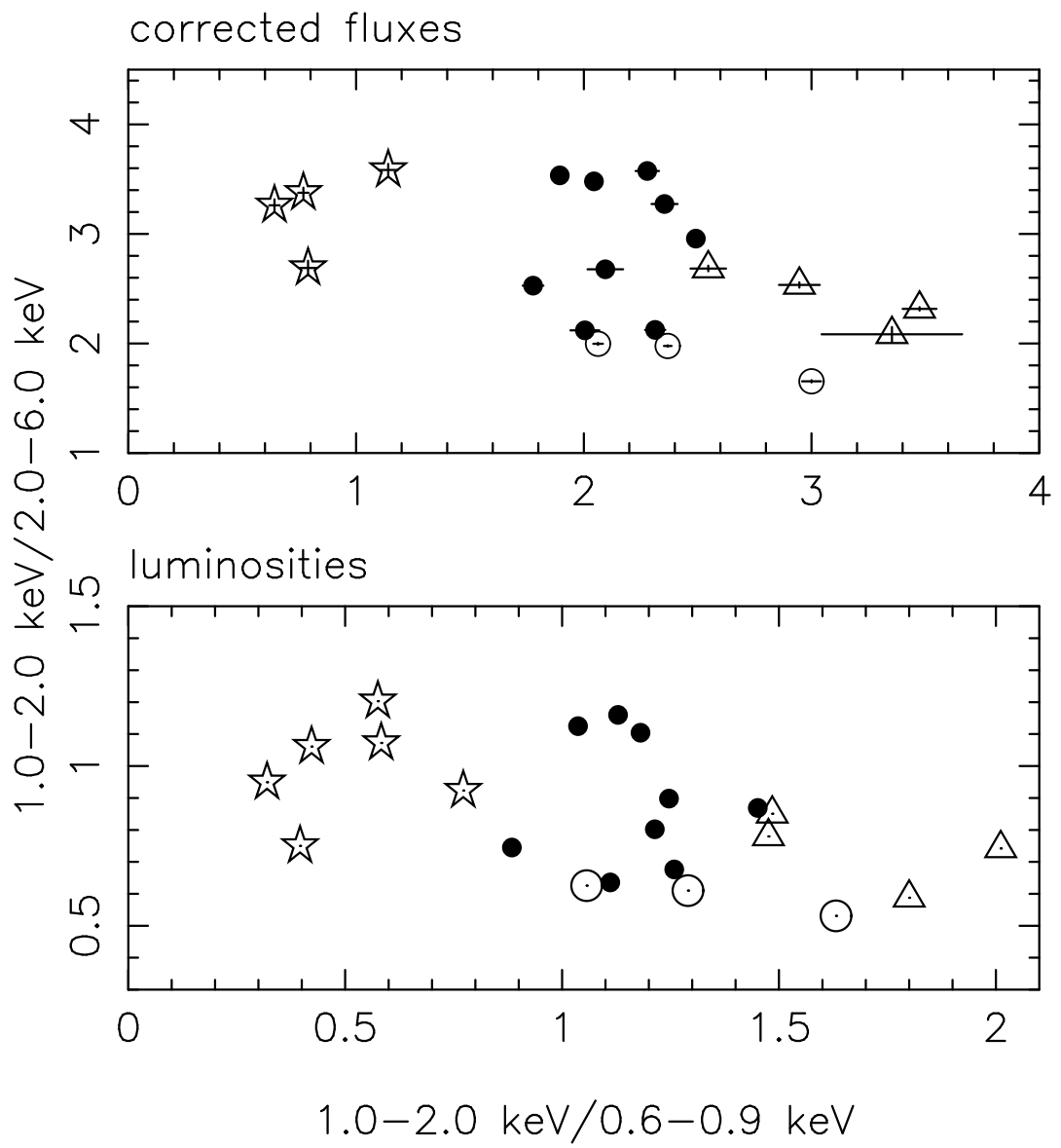

Fig. 3. - Color ratios plots showing the medium (1.0-2.0 keV) to soft $(0.6-0.9 \mathrm{keV})$ ratio on the $\mathrm{x}$ axis, and the medium to hard ratio $(2.0-6.0 \mathrm{keV})$ on the y axis. The objects separate according to the $\mathrm{x}$ axis parameter, the strength of the soft excess. Objects with the strongest soft excesses are marked by stars and are found on the left part of the graph, while objects marked by circles have weaker but still statistically highly significant soft excesses. Mrk 766 and NGC 4051 are distinguished by open circles. Objects marked by triangles have no detected soft excess and are typically absorbed sources. The highly absorbed objects Mrk 507 and IRAS 20181-2244 are off the plot toward the right. Top: ratios from SIS0+SIS1 count rates after correction for Galactic absorption and redshift. The ratios for the highest redshift objects cannot be correctly determined this way, so PHL 1092 and RX J0439-45 are not shown. Bottom: ratios from model luminosities. 

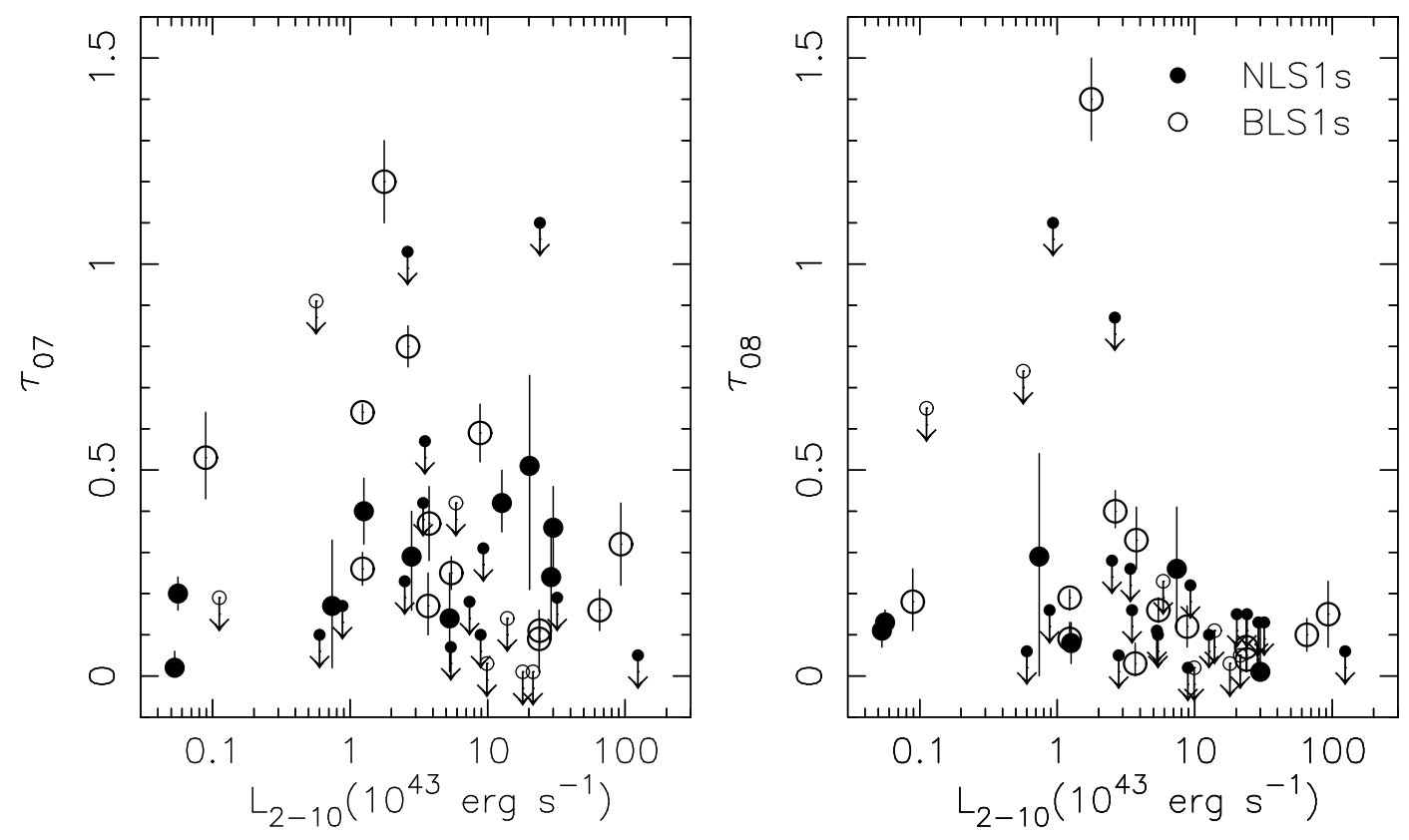

Fig. 4.- The O VII and O VIII optical depths versus luminosity for the narrow-line Seyfert 1 galaxies analyzed in this paper (solid points) and 20 broad-line AGN presented in Reynolds (1997; open points). Note that $3 \mathrm{C} 273\left(L_{x}=1530 \times 10^{43} \mathrm{ergs} \mathrm{s}^{-1}\right)$ is not plotted to preserve the scale. Upper limits are plotted with a smaller version of the same symbol and an arrow. The distributions of $\tau_{O V I I}$ are the same for both NLS1s and broad-line AGN, but the distributions of $\tau_{O V I I I}$ are different at $>97 \%$ confidence. The difference is attributable to a lower optical depth of O VIII in the NLS1s. The left panel indeed shows that the upper limits of many of the NLS1s are smaller than the detections from the broad-line Seyfert 1 s. 


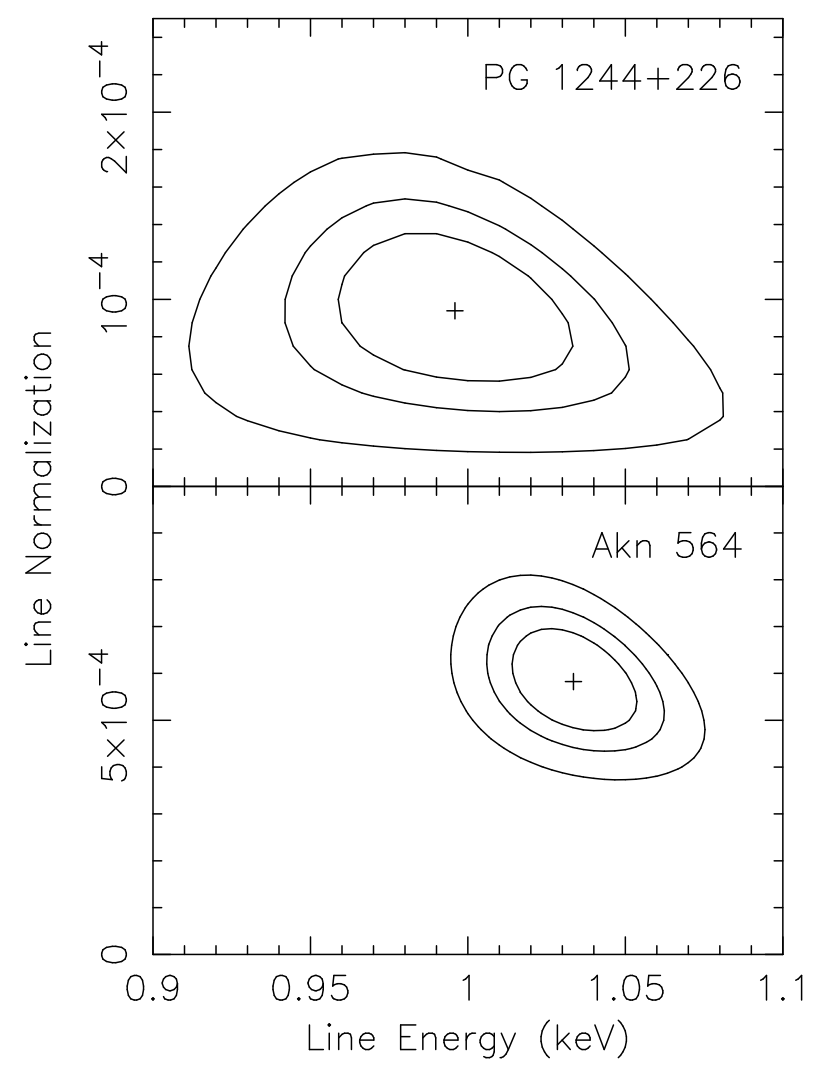

Fig. 5.- $\chi^{2}$ contours $(68 \%, 90 \%$ and $99 \%)$ for the central energy in the rest frame of the object and width $\sigma$ of a Gaussian line model for the soft X-ray line in PG $1244+226$ and Ark 564. The width $\sigma$ has been fixed at its best fit value for stability in calculating the contour. 


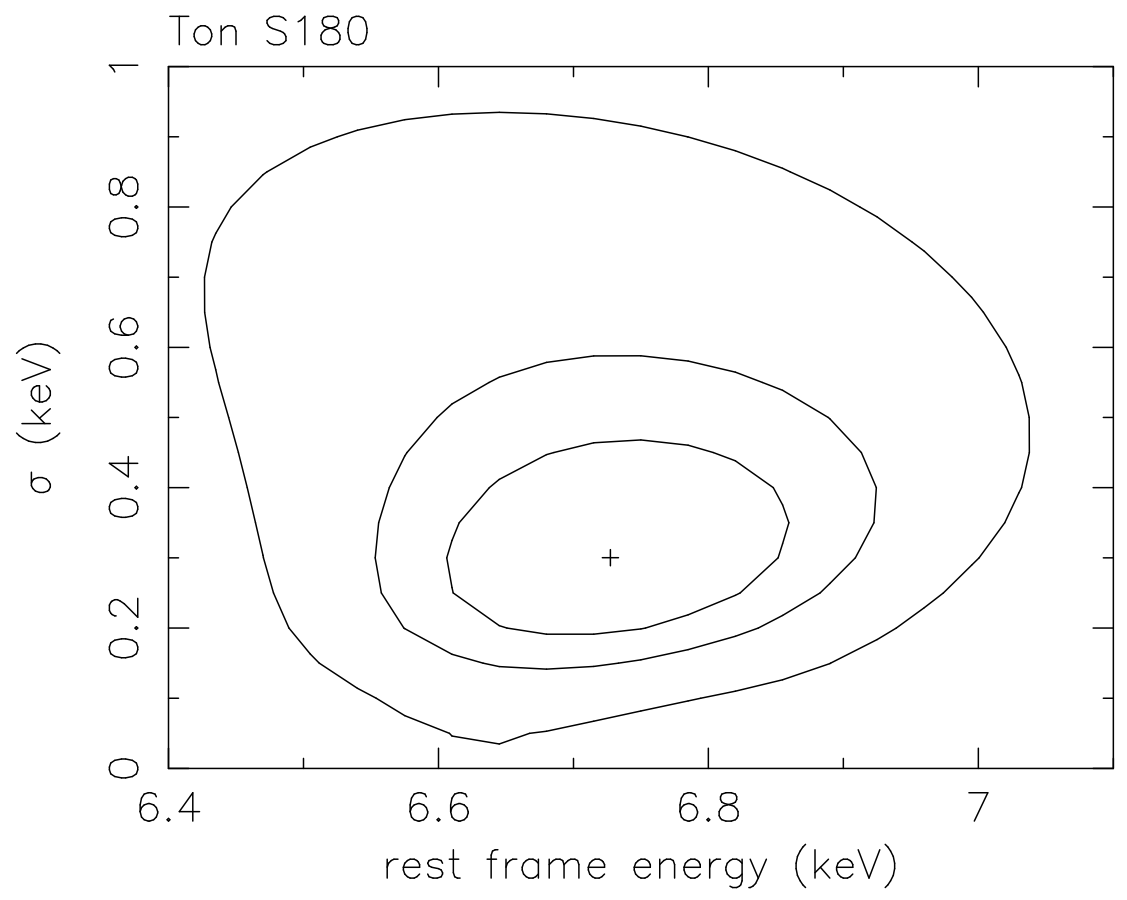

Fig. 6.- $\chi^{2}$ contours $(68 \%, 90 \%$ and $99 \%)$ for the central energy in the rest frame of the object and width $\sigma$ of a Gaussian line model for the iron line in Ton S180. These show that the line is significantly broad and the energy excludes the neutral iron emission energy at $>99 \%$ confidence. 


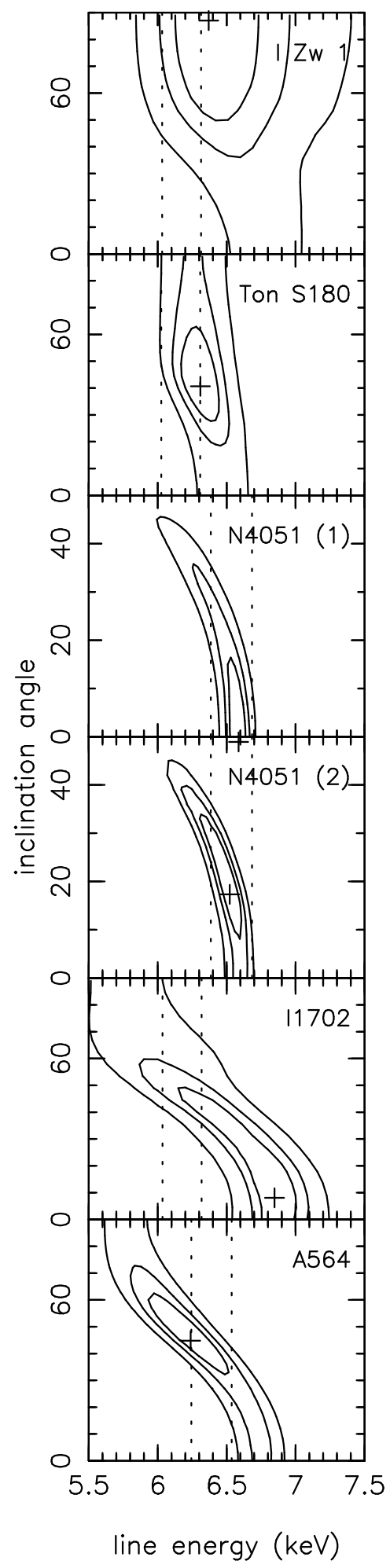

Fig. 7.- Results from fitting a disk line model to 6 spectra from 5 objects in which a significantly broad iron line was detected. The inner and outer radii are fixed at 6 and $1000 R_{g}$ respectively, and the emissivity is fixed at the best fit value between -2 and -3 . The energy is in the observers frame and thus the positions of rest 6.4 and $6.7 \mathrm{keV}$ are shown by dotted lines. 

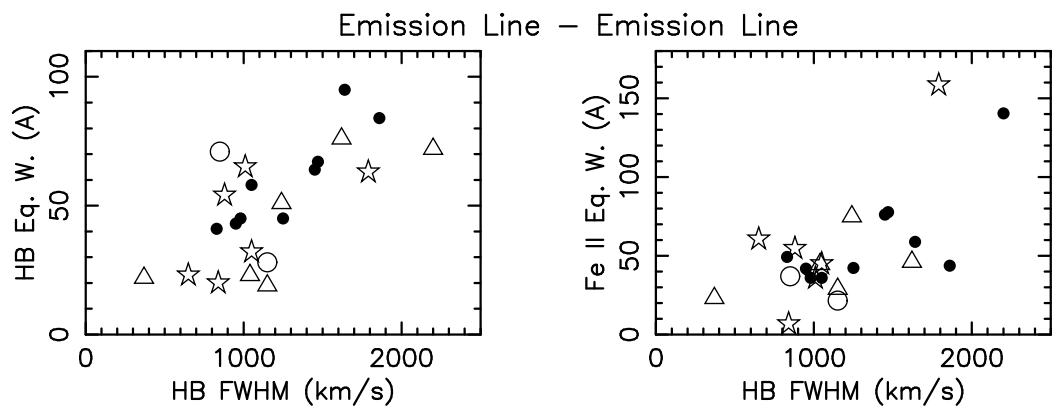

Emission Line - Continuum
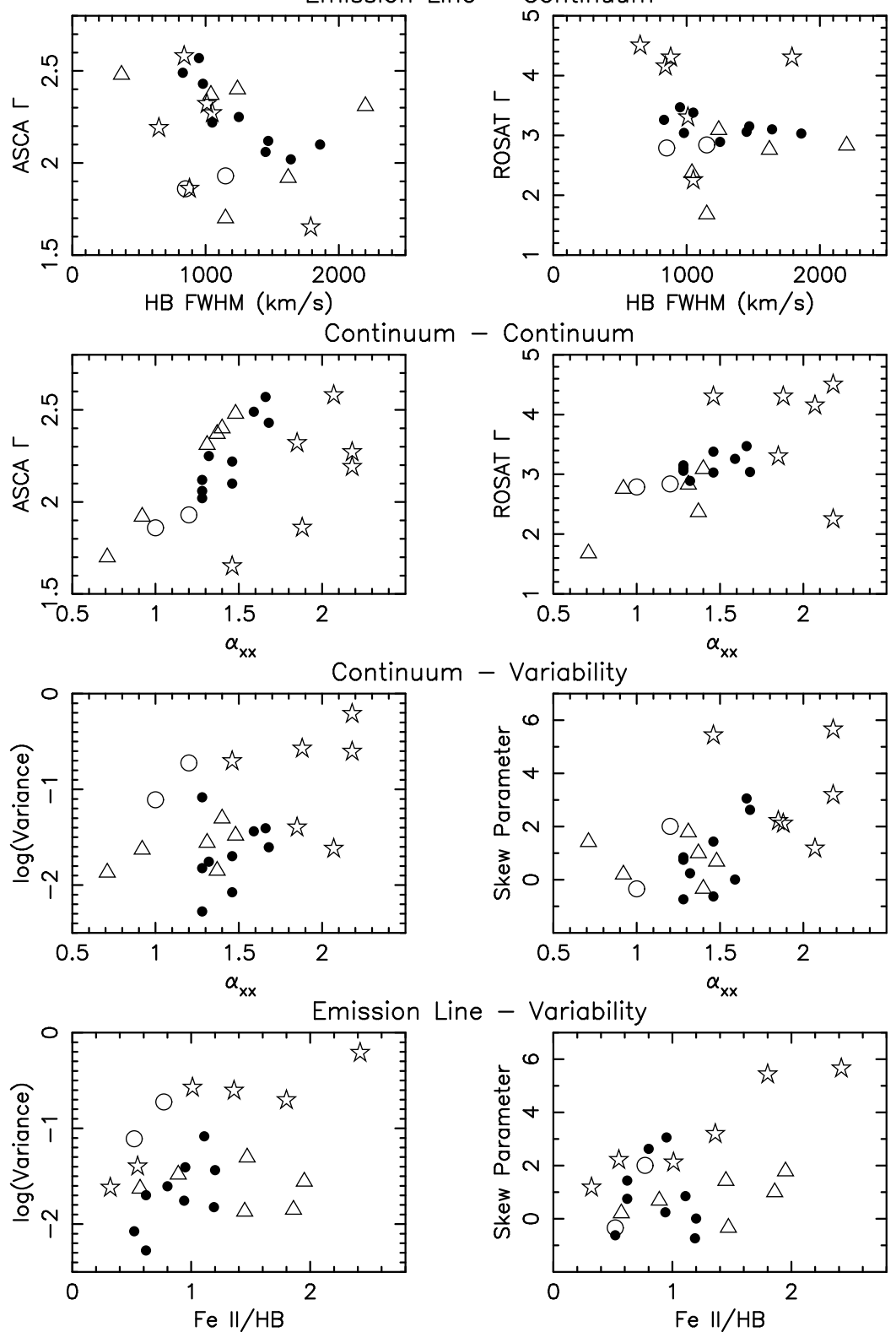

Fig. 8.- Results of correlation analysis. In each panel, the objects are marked with the same symbols as in Figure 3. Stars mark the 6 objects with very strong soft excesses, filled circles mark the 9 objects with weak soft excesses, open circles mark Mrk 766 and NGC 4051, and open triangles mark objects with no soft excesses and which for the most part are absorbed. 
Table 3a. Spectral Fitting Results - Continuum Parameters and Derived Properties

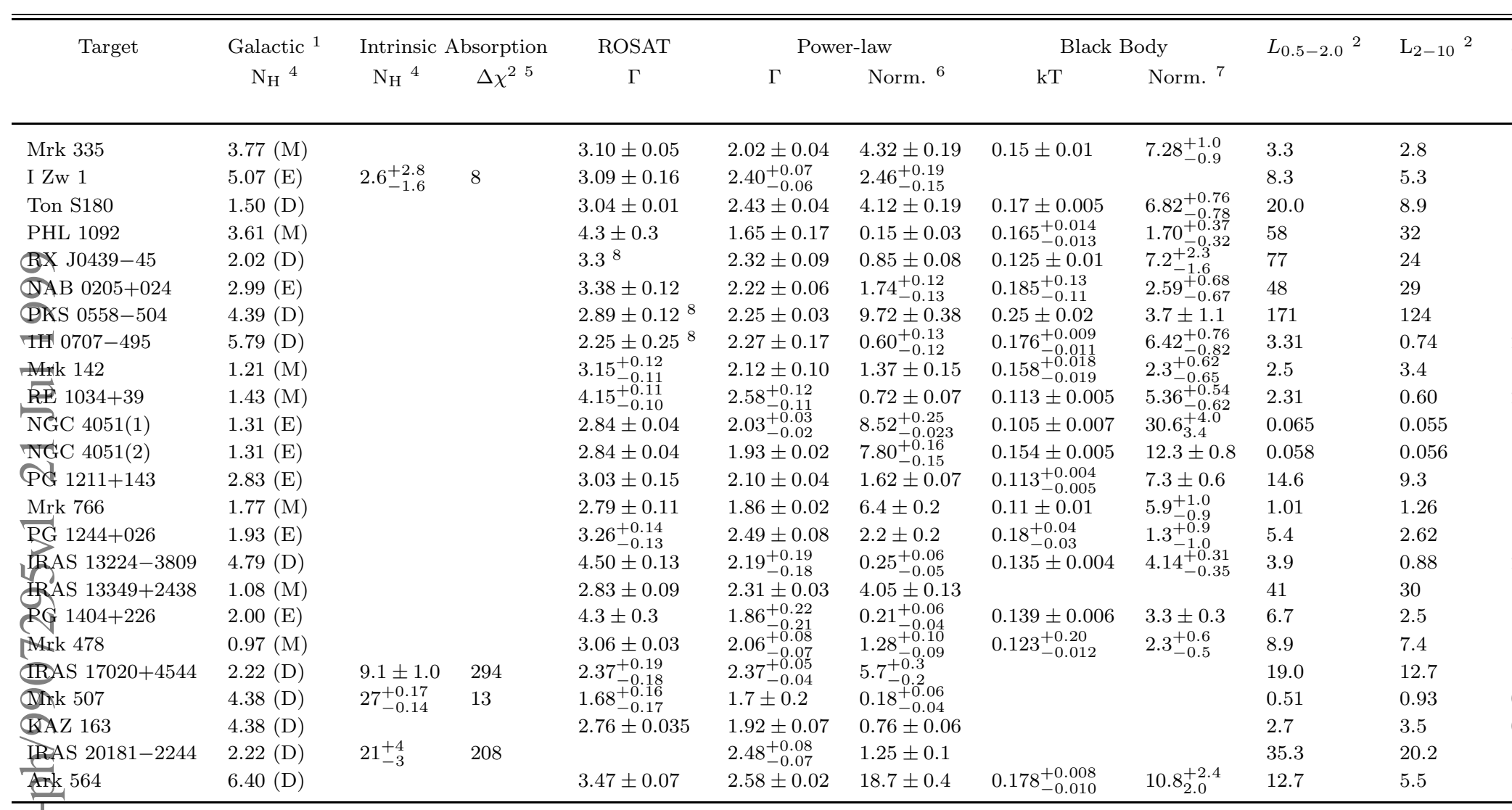

Reference for Galactic $N_{H}$ : (M) Murphy et al. 1996; (E) Elvis, Lockman \& Wilkes 1989; (D) Dickey \& Lockman 1990

22 Thtrinsic luminosity; units of $10^{43} \mathrm{ergs} \mathrm{s}^{-1}$.

3 The black body parameter, defined as the difference in the flux of the black body and power law at $0.6 \mathrm{keV}$ in the rest frame of the object, divided by the su

- ${ }^{4}$ Units of $10^{20} \mathrm{~cm}^{-2}$

5 Difference in $\chi^{2}$ between a model without and with this parameter.

6Units of $10^{-3}$ photons $\mathrm{keV}^{-1} \mathrm{~cm}^{-2}$ at $1 \mathrm{keV}$.

${ }^{7}$ Source luminosity divided by distance squared in units of $10^{-5} \times 10^{39} \mathrm{ergs} \mathrm{s}^{-1} /\left(10^{10} \mathrm{kpc}\right)^{2}$

${ }^{8}$ No pointed ROSAT observation was available. RX J0439-45: the value was taken from Grupe 1996; PKS 0558-504: the value was taken from Schartel et value inferred from the RASSBSC hardness ratios (Grupe 1997, P. comm.) 
Table 3b. Spectral Fitting Results - Oxygen Edge and Iron Line Parameters

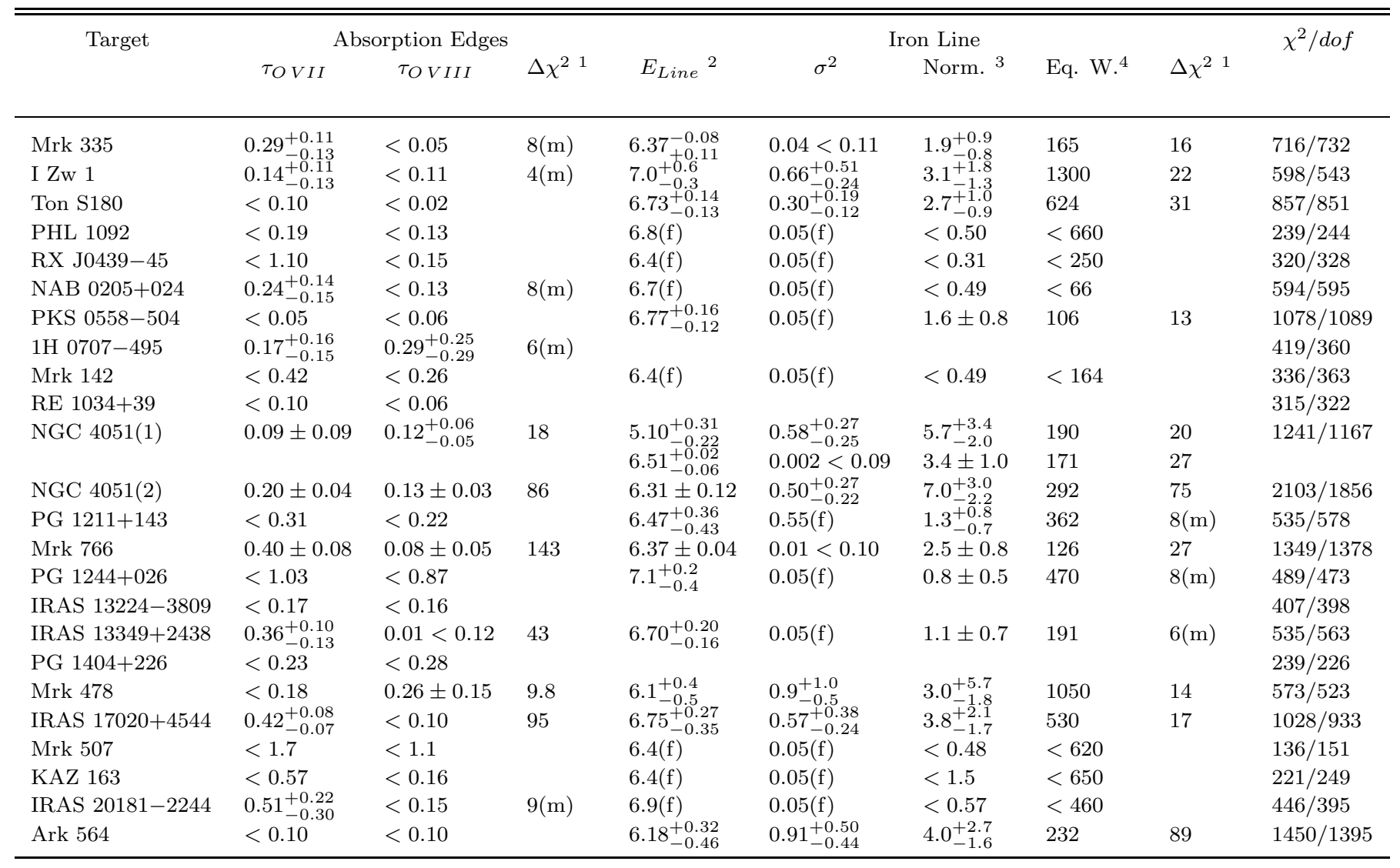

${ }^{1}$ Difference in $\chi^{2}$ between a model without and with this parameter. (m) denotes a marginal detection; see text.

${ }^{2}$ Units of $\mathrm{keV}$.

${ }^{3}$ Units of $10^{-5}$ photons $\mathrm{cm}^{-2} \mathrm{~s}^{-1}$.

${ }^{4} \mathrm{Units}$ of $\mathrm{eV}$. 
Table 3c. Spectral Fitting Results - Soft X-ray Features

\begin{tabular}{|c|c|c|c|c|c|}
\hline \multirow[t]{3}{*}{ Target } & & & & & $\Delta \chi^{2}$ \\
\hline & \multicolumn{4}{|c|}{ ABSORPTION EDGE PARAMETERS: } & \multirow[b]{3}{*}{108} \\
\hline & Edge Energy $^{1}$ & $\tau$ & Edge Energy $^{1}$ & $\tau$ & \\
\hline $1 \mathrm{H} 0707-495$ & $0.95 \pm 0.04$ & $0.54_{-0.25}^{+0.31}$ & $1.10 \pm 0.02$ & $0.78 \pm 0.20$ & \\
\hline IRAS $13224-3809$ & $1.04 \pm 0.04$ & $0.39 \pm 0.20$ & $1.18 \pm 0.03$ & $0.69_{-0.24}^{+0.23}$ & 101 \\
\hline \multirow[t]{3}{*}{ PG $1404+226$} & $1.08 \pm 0.02$ & $0.75_{-0.21}^{+0.22}$ & & & 38 \\
\hline & \multicolumn{4}{|c|}{ SOFT X-RAY LINE PARAMETERS: } & \\
\hline & Line Energy ${ }^{1}$ & $\sigma^{1}$ & Norm. ${ }^{2}$ & Eq. W. ${ }^{3}$ & \\
\hline \multirow{2}{*}{$\begin{array}{l}\text { PG } 1244+026 \\
\text { Ark } 564\end{array}$} & $1.00_{-0.06}^{+0.05}$ & $0.066_{-0.066}^{+0.057}$ & \multirow{2}{*}{$\begin{array}{l}9.5_{-4.9}^{+8.2} \\
58_{-17}^{+48}\end{array}$} & 35 & 14 \\
\hline & $1.033_{-0.031}^{+0.024}$ & $0.10_{-0.03}^{+0.08}$ & & 29 & 78 \\
\hline
\end{tabular}

${ }^{1}$ Units of $\mathrm{keV}$.

${ }^{2}$ Units of $10^{-5}$ photons $\mathrm{cm}^{-2} \mathrm{~s}^{-1}$.

${ }^{3}$ Units of eV. 
Table 5. Correlation Matrix

\begin{tabular}{|c|c|c|c|c|c|c|c|c|c|c|c|c|c|}
\hline \multirow[b]{2}{*}{ Property } & \multirow[b]{2}{*}{$\begin{array}{c}\text { \# of } \\
\text { points }\end{array}$} & \multicolumn{5}{|c|}{ Optical Emission-line Properties } & \multicolumn{5}{|c|}{ X-ray Continuum Properties } & \multicolumn{2}{|c|}{ Soft Excess Properties } \\
\hline & & FWHM & $\begin{array}{c}\mathrm{H} \beta \\
\mathrm{Eq} \cdot \mathrm{W} .\end{array}$ & $\begin{array}{c}\text { Fe II } \\
\text { Eq.W. }\end{array}$ & $\mathrm{Fe} I \mathrm{I} / \mathrm{H} \beta$ & {$[\mathrm{O} \quad \mathrm{III}] / \mathrm{H} \beta$} & $\mathrm{L}_{2-10}$ & $\begin{array}{c}\mathrm{ASCA} \\
\Gamma\end{array}$ & $\begin{array}{c}\text { ROSAT } \\
\Gamma\end{array}$ & $\alpha_{x x}$ & $\begin{array}{l}\text { Color } \\
\text { ratio }\end{array}$ & $\frac{F(b)-F(p)}{F(b)+F(p)}$ & $\mathrm{kT}$ \\
\hline FWHM & $\begin{array}{l}23 \\
17\end{array}$ & & $\begin{array}{l}\bullet 0.003+ \\
\bullet 0.013+\end{array}$ & $\begin{array}{c}\circ 0.016+ \\
0.201+\end{array}$ & $\begin{array}{l}0.692+ \\
0.901-\end{array}$ & $\begin{array}{l}0.060- \\
0.187-\end{array}$ & $\begin{array}{r}0.096+ \\
\circ 0.053+\end{array}$ & $\begin{array}{ll}\circ & 0.023- \\
\circ & 0.039-\end{array}$ & $\begin{array}{l}0.127- \\
0.248-\end{array}$ & $\begin{array}{ll}\circ & 0.017- \\
\circ & 0.056-\end{array}$ & $\begin{array}{l}0.833+ \\
0.303+\end{array}$ & $0.592-$ & $0.901+$ \\
\hline $\mathrm{H} \beta$ Eq.W. & $\begin{array}{l}23 \\
17\end{array}$ & $\begin{array}{ll}\bullet & 0.003+ \\
\bullet & 0.013+\end{array}$ & & $\begin{array}{r}0.016+ \\
0.201+\end{array}$ & $\begin{array}{l}0.132- \\
0.145-\end{array}$ & $\begin{array}{r}0.054- \\
0.283-\end{array}$ & $\begin{array}{l}0.178+ \\
0.091+\end{array}$ & $\begin{array}{ll}\circ & 0.050- \\
\circ & 0.039-\end{array}$ & $\begin{array}{l}0.910- \\
0.409-\end{array}$ & $\begin{array}{r}0.080- \\
\circ 0.020-\end{array}$ & $\begin{array}{l}0.672- \\
0.201+\end{array}$ & $0.837+$ & $0.265-$ \\
\hline Fe II Eq.W. & $\begin{array}{l}23 \\
17\end{array}$ & $\begin{array}{r}0.016+ \\
0.201+\end{array}$ & $\begin{array}{r}\circ 0.016+ \\
0.201+\end{array}$ & & $\begin{array}{l}\bullet 0.006+ \\
\bullet 0.004+\end{array}$ & $\begin{array}{l}0.061- \\
0.343-\end{array}$ & $\begin{array}{l}0.413+ \\
0.564+\end{array}$ & $\begin{array}{r}0.224- \\
\circ 0.043-\end{array}$ & $\begin{array}{l}0.535+ \\
0.433+\end{array}$ & $\begin{array}{l}0.672- \\
0.554-\end{array}$ & $\begin{array}{l}0.139- \\
0.510-\end{array}$ & $0.680-$ & $0.869+$ \\
\hline $\mathrm{Fe} \mathrm{II} / \mathrm{H} \beta$ & $\begin{array}{l}23 \\
17\end{array}$ & $\begin{array}{l}0.692+ \\
0.901-\end{array}$ & $\begin{array}{l}0.132- \\
0.145-\end{array}$ & $\begin{array}{ll}\bullet & 0.006+ \\
\bullet & 0.004+\end{array}$ & & $\begin{array}{l}0.895- \\
0.836-\end{array}$ & $\begin{array}{l}0.812+ \\
0.805-\end{array}$ & $\begin{array}{l}1.000 \\
0.710-\end{array}$ & $\begin{array}{l}1.000 \\
0.265+\end{array}$ & $\begin{array}{l}0.750+ \\
0.339+\end{array}$ & $\begin{array}{l}0.634- \\
0.187-\end{array}$ & $0.621-$ & $0.098+$ \\
\hline$[\mathrm{O} \quad \mathrm{III}] / \mathrm{H} \beta$ & $\begin{array}{l}23 \\
17\end{array}$ & $\begin{array}{l}0.060- \\
0.187-\end{array}$ & $\begin{array}{r}0.054- \\
0.283-\end{array}$ & $\begin{array}{l}0.061- \\
0.343-\end{array}$ & $\begin{array}{l}0.895- \\
0.836-\end{array}$ & & $\begin{array}{l}0.214- \\
0.076-\end{array}$ & $\begin{array}{l}0.711+ \\
0.869+\end{array}$ & $\begin{array}{l}0.821- \\
0.284+\end{array}$ & $\begin{array}{l}0.672- \\
0.803+\end{array}$ & $\begin{array}{r}0.039+ \\
0.650+\end{array}$ & $0.967+$ & $0.536-$ \\
\hline $\mathrm{L}_{2-10}$ & $\begin{array}{l}23 \\
17\end{array}$ & $\begin{array}{c}0.096+ \\
\circ 0.053+\end{array}$ & $\begin{array}{l}0.178+ \\
0.091+\end{array}$ & $\begin{array}{l}0.413+ \\
0.564+\end{array}$ & $\begin{array}{l}0.812+ \\
0.805-\end{array}$ & $\begin{array}{l}0.214- \\
0.076-\end{array}$ & & $\begin{array}{l}0.597+ \\
0.902-\end{array}$ & $\begin{array}{l}1.000 \\
0.837+\end{array}$ & $\begin{array}{l}0.958- \\
0.590+\end{array}$ & $\begin{array}{l}0.958+ \\
0.934+\end{array}$ & $0.410+$ & $0.187+$ \\
\hline ASCA $\Gamma$ & $\begin{array}{l}23 \\
17\end{array}$ & $\begin{array}{ll}\circ & 0.023- \\
\circ & 0.039-\end{array}$ & $\begin{array}{ll}\circ & 0.050- \\
\circ & 0.039-\end{array}$ & $\begin{array}{ll} & 0.224- \\
\circ & 0.043-\end{array}$ & $\begin{array}{l}1.000 \\
0.710-\end{array}$ & $\begin{array}{l}0.711+ \\
0.869+\end{array}$ & $\begin{array}{l}0.597+ \\
0.902-\end{array}$ & & $\begin{array}{l}0.296+ \\
0.509+\end{array}$ & $\begin{array}{ll}\bullet & 0.003+ \\
\circ & 0.025+\end{array}$ & $\begin{array}{l}0.543- \\
0.433-\end{array}$ & $0.149+$ & $0.231+$ \\
\hline ROSAT $\Gamma$ & $\begin{array}{l}22 \\
17\end{array}$ & $\begin{array}{l}0.127- \\
0.248-\end{array}$ & $\begin{array}{l}0.910- \\
0.409-\end{array}$ & $\begin{array}{l}0.535+ \\
0.433+\end{array}$ & $\begin{array}{l}1.000 \\
0.265+\end{array}$ & $\begin{array}{l}0.821- \\
0.248+\end{array}$ & $\begin{array}{l}1.000 \\
0.837+\end{array}$ & $\begin{array}{l}0.296+ \\
0.509+\end{array}$ & & $\begin{array}{ll}\bullet & 0.002+ \\
\circ & 0.020+\end{array}$ & $\begin{array}{ll}\bullet & 0.002- \\
\circ & 0.019-\end{array}$ & $0.108-$ & $0.967-$ \\
\hline$\alpha_{x x}$ & $\begin{array}{l}23 \\
17\end{array}$ & $\begin{array}{ll}\circ & 0.017- \\
\circ & 0.056-\end{array}$ & $\begin{array}{r}0.080- \\
\circ 0.020-\end{array}$ & $\begin{array}{l}0.672- \\
0.554-\end{array}$ & $\begin{array}{l}0.750+ \\
0.339+\end{array}$ & $\begin{array}{l}0.672- \\
0.803+\end{array}$ & $\begin{array}{l}0.958- \\
0.590+\end{array}$ & $\begin{array}{ll}\bullet & 0.003+ \\
\circ & 0.025+\end{array}$ & $\begin{array}{ll}\bullet & 0.002+ \\
\circ & 0.020+\end{array}$ & & $\begin{array}{l}\bullet 0.001- \\
\bullet 0.001-\end{array}$ & $0.062-$ & $0.835+$ \\
\hline Color ratio & $\begin{array}{l}23 \\
17\end{array}$ & $\begin{array}{l}0.833+ \\
0.303+\end{array}$ & $\begin{array}{l}0.672- \\
0.201+\end{array}$ & $\begin{array}{l}0.139- \\
0.510-\end{array}$ & $\begin{array}{l}0.634- \\
0.187-\end{array}$ & $\begin{array}{r}0.039+ \\
0.650+\end{array}$ & $\begin{array}{l}0.958+ \\
0.934+\end{array}$ & $\begin{array}{l}0.543- \\
0.433-\end{array}$ & $\begin{array}{ll}\bullet & 0.002- \\
\circ & 0.019-\end{array}$ & $\begin{array}{l}\bullet 0.001- \\
\bullet 0.001-\end{array}$ & & $\bullet 0.001+$ & $0.283+$ \\
\hline \multicolumn{14}{|l|}{$\frac{F(b)-F(p)}{F(b)+F(p)}$} \\
\hline $\mathrm{kT}$ & 17 & $0.901+$ & $0.265-$ & $0.869+$ & $0.098+$ & $0.536-$ & $0.187+$ & $0.231+$ & $0.967-$ & $0.835+$ & $0.283+$ & $0.083-$ & \\
\hline $\begin{array}{l}\text { Excess } \\
\text { Variance }\end{array}$ & $\begin{array}{l}23 \\
17\end{array}$ & $\begin{array}{l}0.107- \\
0.091-\end{array}$ & $\begin{array}{l}0.443- \\
0.076-\end{array}$ & $\begin{array}{l}0.544+ \\
0.742+\end{array}$ & $\begin{array}{l}0.214+ \\
\circ 0.039+\end{array}$ & $\begin{array}{l}0.616+ \\
0.387+\end{array}$ & $\begin{array}{l}0.119- \\
0.084-\end{array}$ & $\begin{array}{l}0.833- \\
0.483-\end{array}$ & $\begin{array}{l}0.080+ \\
0.303+\end{array}$ & $\begin{array}{r}0.039+ \\
0.089+\end{array}$ & $\begin{array}{r}0.027- \\
0.070-\end{array}$ & $\circ 0.039-$ & $0.805+$ \\
\hline $\begin{array}{l}\text { Skew } \\
\text { Parameter }\end{array}$ & $\begin{array}{l}23 \\
17\end{array}$ & $\begin{array}{l}0.279- \\
0.303-\end{array}$ & $\begin{array}{r}0.107- \\
\circ 0.053-\end{array}$ & $\begin{array}{l}0.731- \\
1.000+\end{array}$ & $\begin{array}{r}0.119+ \\
\circ 0.058+\end{array}$ & $\begin{array}{l}0.895+ \\
0.536+\end{array}$ & $\begin{array}{l}0.692- \\
0.680-\end{array}$ & $\begin{array}{l}0.833+ \\
0.650+\end{array}$ & $\begin{aligned} & 0.091+ \\
\circ & 0.053+\end{aligned}$ & $\begin{array}{ll}\circ & 0.020+ \\
\circ & 0.023+\end{array}$ & $\begin{array}{l}\circ 0.020- \\
\circ 0.032-\end{array}$ & $\circ 0.058-$ & $0.409+$ \\
\hline$\tau_{O V I I}$ & $\begin{array}{l}23 \\
17\end{array}$ & $\begin{array}{l}0.831+ \\
0.664+\end{array}$ & $\begin{array}{l}0.831+ \\
0.128+\end{array}$ & $\begin{array}{l}0.550- \\
0.613-\end{array}$ & $\begin{aligned} & 0.669+ \\
\circ & 0.036-\end{aligned}$ & $\begin{array}{l}0.073+ \\
0.277+\end{array}$ & $\begin{array}{l}0.608+ \\
0.426-\end{array}$ & $\begin{array}{l}0.966- \\
0.111-\end{array}$ & $\begin{array}{r}0.012- \\
0.096-\end{array}$ & $\begin{aligned} & 0.113- \\
\circ & 0.041-\end{aligned}$ & $\begin{array}{ll}\bullet & 0.010+ \\
\circ & 0.036+\end{array}$ & $0.515-$ & $0.514-$ \\
\hline$\tau_{O V I I I}$ & $\begin{array}{l}23 \\
17\end{array}$ & $\begin{array}{l}0.869- \\
0.713+\end{array}$ & $\begin{array}{l}0.583+ \\
0.461-\end{array}$ & $\begin{array}{l}0.742+ \\
0.659+\end{array}$ & $\begin{array}{l}0.510+ \\
0.185+\end{array}$ & $\begin{array}{l}0.660- \\
0.883+\end{array}$ & $\begin{array}{l}0.099- \\
0.141-\end{array}$ & $\begin{array}{l}0.323- \\
0.659-\end{array}$ & $\begin{array}{r}0.109- \\
\circ 0.039-\end{array}$ & $\begin{array}{l}0.700- \\
0.824-\end{array}$ & $\begin{array}{l}0.442- \\
0.769-\end{array}$ & $0.659-$ & $0.768-$ \\
\hline
\end{tabular}

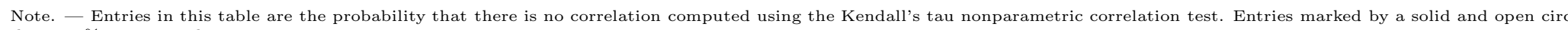
and $\approx>95 \%$, respectively. 